\title{
On Classical Solutions of Linear Stochastic Integro-Differential Equations
}

October 15,2018

\section{James-Michael Leahy}

The University of Edinburgh, E-mail: J.Leahy-2@sms.ed.ac.uk

\section{Remigijus Mikulevičius}

The University of Southern California, E-mail: mikulves@math.usc.edu

\begin{abstract}
We prove the existence of classical solutions to parabolic linear stochastic integrodifferential equations with adapted coefficients using Feynman-Kac transformations, conditioning, and the interlacing of space-inverses of stochastic flows associated with the equations. The equations are forward and the derivation of existence does not use the "general theory" of SPDEs. Uniqueness is proved in the class of classical solutions with polynomial growth.
\end{abstract}

\section{Contents}

1 Introduction $\quad 2$

2 Outline of main results $\quad 4$

3 Proof of main theorems $\quad 11$

3.1 Proof of uniqueness for Theorem 2.2 11

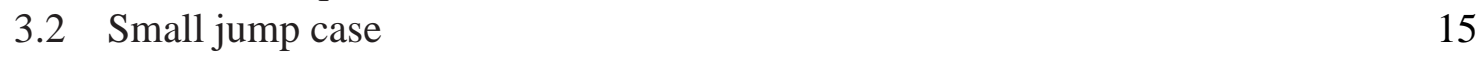

3.3 Adding free and zero-order terms 19

3.4 Adding uncorrelated part (Proof of Theorem 2.2) 24

3.5 Interlacing a sequence of large jumps (Proof of Theorem 2.5) 26

4 Appendix $\quad 29$

4.1 Martingale and point measure measure moment estimates 29

$\begin{array}{lll}4.2 & \text { Optional projection } & 31\end{array}$

4.3 Estimates of Hölder continuous functions 31

4.4 Stochastic Fubini thoerem 41

4.5 Itô-Wentzell formula 43 


\section{Introduction}

Let $(\Omega, \mathcal{F}, \mathbf{P})$ be a complete filtered probability space and $\tilde{\mathcal{F}}_{0}$ be a sub-sigma-algebra of $\mathcal{F}$. We assume that this probability space supports a sequence $\left(w_{t}^{1 ; \varrho}\right)_{\varrho \geq 1}, t \geq 0, \varrho \in \mathbf{N}$, of independent one-dimensional Wiener processes and a Poisson random measure $p^{1}(d t, d z)$ on $\left(\mathbf{R}_{+} \times Z^{1}, \mathcal{B}\left(\mathbf{R}_{+} \otimes \mathcal{Z}^{1}\right)\right.$ with intensity measure $\pi^{1}(d z) d t$, where $\left(Z^{1}, \mathcal{Z}^{1}, \pi^{1}\right)$ is a sigma-finite measure space. We also assume that $\left(w_{t}^{1 ; \varrho}\right)_{\varrho \geq 1}$ and $p^{1}(d t, d z)$ are independent of $\mathcal{F}_{0}$. Let $\mathbf{F}=\left(\mathcal{F}_{t}\right)_{t \geq 0}$ be the standard augmentation of the filtration $\left(\overline{\mathcal{F}}_{t}\right)_{t \geq 0}$, where for each $t \geq 0$,

$$
\overline{\mathcal{F}}_{t}=\sigma\left(\tilde{\mathcal{F}}_{0},\left(w_{s}^{1}\right)_{\varrho \geq 1}, p^{1}([0, s], \Gamma): s \leq t, \Gamma \in \mathcal{Z}^{1}\right) .
$$

For each real number $T>0$, we let $\mathcal{R}_{T}, \mathcal{O}_{T}$, and $\mathcal{P}_{T}$ be the $\mathbf{F}$-progressive, F-optional, and F-predictable sigma-algebra on $\Omega \times[0, T]$, respectively. Denote by $q^{1}(d t, d z)=p^{1}(d t, d z)-$ $\pi^{1}(d z) d t$ the compensated Poisson random measure. Let $D^{1}, E^{1}, V^{1} \in Z$ be disjoint $Z^{1}$ measurable subsets such that $D^{1} \cup E^{1} \cup V^{1}=Z^{1}$ and $\pi\left(V^{1}\right)<\infty$. Let $\left(Z^{2}, Z^{2}, \pi^{2}\right)$ be a sigma-finite measure space and $D^{2}, E^{2} \in Z^{2}$ be disjoint $Z^{2}$-measurable subsets such that $D^{2} \cup E^{2}=Z^{2}$.

Fix an arbitrary positive real number $T>0$ and integers $d_{1}, d_{2} \geq 1$. Let $\alpha \in(0,2]$ and let $\tau \leq T$ be a stopping time. Let $\mathcal{F}_{\tau}$ be the stopping time sigma-algebra associated with $\tau$ and let $\varphi: \Omega \times \mathbf{R}^{d_{1}} \rightarrow \mathbf{R}^{d_{2}}$ be $\mathcal{F}_{\tau} \otimes \mathcal{B}\left(\mathbf{R}^{d_{1}}\right)$-measurable. We consider the system of stochastic integro-differential equations on $[0, T] \times \mathbf{R}^{d_{1}}$ given by

$$
\begin{aligned}
d u_{t}^{l}= & \left(\left(\mathcal{L}_{t}^{1 ; l}+\mathcal{L}_{t}^{2 ; l}\right) u_{t}+\mathbf{1}_{[1,2]}(\alpha) b_{t}^{i} \partial_{i} u_{t}^{l}+c_{t}^{l \bar{l}} u_{t}^{\bar{l}}+f_{t}^{l}\right) d t+\left(\mathcal{N}_{t}^{1 ; l \varrho} u_{t}+g_{t}^{l \varrho}\right) d w_{t}^{1 ; \varrho} \\
& +\int_{Z^{1}}\left(\mathcal{I}_{t, z}^{1 ; l} u_{t-}+h_{t}^{l}(z)\right)\left[\mathbf{1}_{D^{1}}(z) q^{1}(d t, d z)+\mathbf{1}_{E^{1} \cup V^{1}}(z) p^{1}(d t, d z)\right], \quad \tau \leq t \leq T, \\
u_{t}^{l}= & \varphi^{l}, \quad t \leq \tau, \quad l \in\left\{1, \ldots, d_{2}\right\},
\end{aligned}
$$

where for $\phi \in C_{c}^{\infty}\left(\mathbf{R}^{d_{1}} ; \mathbf{R}^{d_{2}}\right), k \in\{1,2\}$, and $l \in\left\{1, \ldots, d_{2}\right\}$,

$$
\begin{aligned}
\mathcal{L}_{t}^{k ; l} \phi(x):= & \mathbf{1}_{\{2\}}(\alpha) \frac{1}{2} \sigma_{t}^{k ; i \varrho}(x) \sigma_{t}^{k ; j \varrho}(x) \partial_{i j} \phi^{l}(x)+\mathbf{1}_{\{2\}}(\alpha) \sigma_{t}^{k ; \varrho}(x) v_{t}^{k ; \bar{l} \varrho}(x) \partial_{i} \phi^{\bar{l}}(x) \\
& +\int_{D^{k}} \rho_{t}^{k ; l \bar{l}}(x, z)\left(\phi^{\bar{l}}\left(x+H_{t}^{k}(x, z)\right)-\phi^{\bar{l}}(x)\right) \pi^{k}(d z) \\
& +\int_{D^{k}}\left(\phi^{l}\left(x+H_{t}^{k}(x, z)\right)-\phi^{l}(x)-\mathbf{1}_{(1,2]}(\alpha) H_{t}^{k ; i}(x, z) \partial_{i} \phi^{l}(x)\right) \pi^{k}(d z) \\
& +\mathbf{1}_{\{2\}}(k) \int_{E^{2}}\left(\left(I_{d_{2}}^{l \bar{l}}+\rho_{t}^{2 ; l \bar{l}}(x, z)\right) \phi^{\bar{l}}\left(x+H_{t}^{2}(x, z)\right)-\phi^{l}(x)\right) \pi^{2}(d z), \\
\mathcal{N}_{t}^{1 ; l \varrho} \phi(x):= & \mathbf{1}_{\{2\}}(\alpha) \sigma_{t}^{1 ; l \varrho}(x) \partial_{i} \phi^{l}(x)+v_{t}^{1 ; i \bar{l} \varrho}(x) \phi^{\bar{l}}(x), \varrho \geq 1, \\
\mathcal{I}_{t, z}^{1 ; l} \phi(x):= & \left(I_{d_{2}}^{l \bar{l}}+\rho_{t}^{1 ; l \bar{l}}(x, z)\right) \phi^{\bar{l}}\left(x+H_{t}^{1}(x, z)\right)-\phi^{l}(x),
\end{aligned}
$$

and

$$
\int_{D^{k}}\left(\left|H_{t}^{k}(x, z)\right|^{\alpha}+\left|\rho_{t}^{k}(x, z)\right|^{2}\right) \pi^{k}(d z)+\int_{E^{k}}\left(\left|H_{t}^{k}(x, z)\right|^{1 \wedge \alpha}+\left|\rho_{t}^{k}(x, z)\right|\right) \pi^{k}(d z)<\infty
$$


The summation convention with respect to repeated indices $i, j \in\left\{1, \ldots, d_{1}\right\}, \bar{l} \in\left\{1, \ldots, d_{2}\right\}$, and $\varrho \in \mathbf{N}$ is used here and below. The $d_{2} \times d_{2}$ dimensional identity matrix is denoted by $I_{d_{2}}$. For a subset $A$ of a larger set $X, \mathbf{1}_{A}$ denotes the $\{0,1\}$-valued function taking the value 1 on the set $A$ and 0 on the complement of $A$. We assume that for each $k \in\{1,2\}$,

$$
\begin{gathered}
\sigma_{t}^{k}(x)=\left(\sigma_{t}^{k ; \varrho \varrho}(\omega, x)\right)_{1 \leq i \leq d_{1}, \varrho \geq 1}, \quad b_{t}(x)=\left(b_{t}^{i}(\omega, x)\right)_{1 \leq i \leq d_{1}}, \quad c_{t}(x)=\left(c_{t}^{l \bar{l}}(\omega, x)\right)_{1 \leq l, \bar{l} \leq d_{2}}, \\
v_{t}^{k}(x)=\left(v_{t}^{k ; l \bar{l} \varrho}(\omega, x)\right)_{1 \leq l, \bar{l} \leq d_{2}, \varrho \geq 1}, \quad f_{t}(x)=\left(f_{t}^{i}(\omega, x)\right)_{1 \leq i \leq d_{2}}, \quad g_{t}(x)=\left(g_{t}^{i \varrho}(\omega, x)\right)_{1 \leq i \leq d_{2}, \varrho \geq 1},
\end{gathered}
$$

are random fields on $\Omega \times[0, T] \times \mathbf{R}^{d_{1}}$ that are $\mathcal{R}_{T} \otimes \mathcal{B}\left(\mathbf{R}^{d_{1}}\right)$-measurable. Moreover, for each $k \in\{1,2\}$, we assume that

$$
H_{t}^{k}(x, z)=\left(H_{t}^{k ; i}(\omega, x, z)\right)_{1 \leq i \leq d_{1}}, \rho_{t}^{k}(x, z)=\left(\rho_{t}^{k ; l \bar{l}}(\omega, x, z)\right)_{1 \leq l, \bar{l} \leq d_{2}},
$$

are random fields on $\Omega \times[0, T] \times \mathbf{R}^{d_{1}} \times Z^{k}$ that are $\mathcal{P}_{T} \otimes \mathcal{B}\left(\mathbf{R}^{d_{1}}\right) \otimes \mathcal{Z}^{k}$-measurable. Moreover, we assume that

$$
h_{t}(x, z)=\left(h_{t}^{i}(\omega, x, z)\right)_{1 \leq i \leq d_{2}},
$$

is a random field on $\Omega \times[0, T] \times \mathbf{R}^{d_{1}}$ that is $\mathcal{P}_{T} \otimes \mathcal{B}\left(\mathbf{R}^{d_{1}}\right)$-measurable

Systems of linear stochastic integro-differential equations appear in many contexts. They may be considered as extensions of both first-order symmetric hyperbolic systems and linear fractional advection-diffusion equations. The equation (1.1) also arises in non-linear filtering of semimartingales as the equation for the unormalized filter of the signal (see, e.g., [Gri76] and [GM11]). Moreover, (1.1) is intimately related to linear transformations of inverse flows of jump SDEs and it is precisely this connection that we will exploit to obtain solutions.

There are various techniques available to derive the existence and uniqueness of classical solutions of linear parabolic SPDEs and SIDEs. One approach is to develop a theory of weak solutions for the equations (e.g. variational, mild solution, or etc...) and then study further regularity in classical function spaces via an embedding theorem. We refer the reader to [Par72, Par75, MP76, KR77, Tin77, Gyö82, Wal86, DPZ92, Kry99, CK10, PZ07, Hau05, RZ07, BvNVW08, HØUZ10, LM14a] for more information about weak solutions of SPDEs driven by continuous and discontinuous martingales and martingale measures. This approach is especially important in the non-degenerate setting where some smoothing occurs and has the obvious advantage that it is broader in scope. Another approach is to regard the solution as a function with values in a probability space and use the method deterministic PDEs (i.e. Schauder estimates, see, e.g. [Mik00, MP09]). A third approach is a direct one that uses solutions of stochastic differential equations. The direct method allows to obtain classical solutions in the entire Hölder scale while not restricting to integer derivative assumptions for the coefficients and data.

In this paper, we derive the existence of a classical solutions of (1.1) with regular coefficients using a Feynman-Kac-type transformation and the interlacing of the space-inverse (first integrals [KR81]) of a stochastic flow associated with the equation. The construction of the solution gives an insight into the structure of the solution as well. We prove that the solution of (1.1) is unique in the class of classical solutions with polynomial growth 
(i.e. weighted Hölder spaces). As an immediate corollary of our main result, we obtain the existence and uniqueness of classical solutions of linear integro-differential equations with random coefficients, since the coefficients $\sigma^{1}, H^{1}, a^{1}, \rho^{1}$, and free terms $g$ and $h$ can be zero. Our work here directly extends the method of characteristics for deterministic first-order partial differential equations and the well-known Feynman-Kac formula for deterministic second-order PDEs.

In the continuous case (i.e. $H^{1} \equiv 0, H^{2} \equiv 0, h \equiv 0$ ), the classical solution of (1.1) was constructed in [KR81, Kun81, Kun86, Roz90] (see references therein as well) using the first integrals of the associated backward SDE. This method was also used to obtain classical solutions of (1.1) in [DPMT07]. In the references above, the forward Liouville equation for the first integrals of associated stochastic flow was derived directly. However, since the backward equation involves a time reversal, the coefficients and input functions are assumed to be non-random. The generalized solutions of (1.1) with $d_{2}=1$, non-random coefficients, non-degenerate diffusion, and finite measures $\pi^{1}=\pi^{2}$ were discussed in [MB07]. In this paper, we give a direct derivation of (1.1) and all the equations considered are forward, possibly degenerate, and the coefficients and input functions are adapted. For other interesting and related developments, we refer the reader to [Pri12, Zha13, Pri14].

This paper is organized as follows. In Section 2, our notation is set forth and the main results are stated. In Section 3, the main theorem is proved and is divided into a proof of uniqueness and existence. In Section 4, the appendix, auxiliary facts that are used throughout the paper are discussed.

\section{Outline of main results}

For each integer $n \geq 1$, let $\mathbf{R}^{n}$ be the space of $d$-dimensional Euclidean points $x=\left(x^{1}, \ldots\right.$, $\left.x^{n}\right)$. For each $x$, denote by $|x|$ the Euclidean norm of $x$. Let $\mathbf{R}_{+}$denote the set of non-negative real-numbers. Let $\mathbf{N}$ be the set of natural numbers. Elements of $\mathbf{R}^{d_{1}}$ and $\mathbf{R}^{d_{2}}$ are understood as column vectors and elements of $\mathbf{R}^{2 d_{1}}$ and $\mathbf{R}^{2 d_{2}}$ are understood as matrices of dimension $d_{1} \times d_{1}$ and $d_{2} \times d_{2}$, respectively. For each integer $n \geq 1$, the norm of an element $x$ of $\ell_{2}\left(\mathbf{R}^{n}\right)$, the space of square-summable $\mathbf{R}^{n}$-valued sequences, is denoted by $|x|$. For a topological space $(X, \mathcal{X})$ we denote the Borel sigma-field on $X$ by $\mathcal{B}(X)$.

For each $i \in\left\{1, \ldots, d_{1}\right\}$, let $\partial_{i}=\frac{\partial}{\partial x_{i}}$ be the spatial derivative operator with respect to $x_{i}$ and write $\partial_{i j}=\partial_{i} \partial_{j}$ for each $i, j \in\left\{1, \ldots, d_{1}\right\}$. For a once differentiable function $f=$ $\left(f^{1} \ldots, f^{d_{1}}\right): \mathbf{R}^{d_{1}} \rightarrow \mathbf{R}^{d_{1}}$, we denote the gradient of $f$ by $\nabla f=\left(\partial_{j} f^{i}\right)_{1 \leq i, j \leq d_{1}}$. Similarly, for a once differentiable function $f=\left(f^{1 \varrho}, \ldots, f^{d \varrho}\right)_{\varrho \geq 1}: \mathbf{R}^{d_{1}} \rightarrow \ell_{2}\left(\mathbf{R}^{d_{1}}\right)$, we denote the gradient of $f$ by $\nabla f=\left(\partial_{j} f^{i \varrho}\right)_{1 \leq i, j \leq d_{1}, \varrho \geq 1}$ and understand it as a function from $\mathbf{R}^{d_{1}}$ to $\ell_{2}\left(\mathbf{R}^{2 d_{1}}\right)$. For a multi-index $\gamma=\left(\gamma_{1}, \ldots, \gamma_{d}\right) \in\{0,1,2, \ldots,\}^{d_{1}}$ of length $|\gamma|:=\gamma_{1}+\cdots+\gamma_{d}$, denote by $\partial^{\gamma}$ the operator $\partial^{\gamma}=\partial_{1}^{\gamma_{1}} \cdots \partial_{d}^{\gamma_{d}}$, where $\partial_{i}^{0}$ is the identity operator for all $i \in\left\{1, \ldots, d_{1}\right\}$. For each integer $d \geq 1$, we denote by $C_{c}^{\infty}\left(\mathbf{R}^{d_{1}} ; \mathbf{R}^{d}\right)$ the space of infinitely differentiable functions with compact support in $\mathbf{R}^{d}$.

For a Banach space $V$ with norm $|\cdot|_{V}$, domain $Q$ of $\mathbf{R}^{d}$, and continuous function $f: Q \rightarrow$ 
$V$, we define

$$
|f|_{0 ; Q ; V}=\sup _{x \in Q}|f(x)|
$$

and

$$
[f]_{\beta ; Q ; V}=\sup _{x, y \in Q, x \neq y} \frac{|f(x)-f(y)|_{V}}{|x-y|_{V}^{\beta}}, \beta \in(0,1] .
$$

For each real number $\beta \in \mathbf{R}$, we write $\beta=[\beta]^{-}+\{\beta\}^{+}$, and $\{\beta\}^{+} \in(0,1]$. For a Banach space $V$ with norm $|\cdot|_{V}$, real number $\beta>0$, and domain $Q$ of $\mathbf{R}^{d}$, we denote by $C^{\beta}(Q ; V)$ the Banach space of all bounded continuous functions $f: Q \rightarrow V$ having finite norm

$$
|f|_{\beta ; Q ; V}:=\sum_{|\gamma| \leq[\beta]^{-}}\left|\partial^{\gamma} f\right|_{0 ; Q ; V}+\sum_{|\gamma|=[\beta]^{-}}\left[\partial^{\gamma} f\right]_{\{\beta\}^{+} ; Q ; V} .
$$

When $Q=\mathbf{R}^{d_{1}}$ and $V=\mathbf{R}^{n}$ or $V=\ell_{2}\left(\mathbf{R}^{n}\right)$ for any integer $n \geq 1$, we drop the subscripts $Q$ and $V$ from the norm $|\cdot|_{\beta ; Q ; V}$ and write $|\cdot|_{\beta}$. For a Banach space $V$ and for each $\beta>0$, denote by $C_{l o c}^{\beta}\left(\mathbf{R}^{d} ; V\right)$ the Fréchet space of continuous functions $f: \mathbf{R}^{d} \rightarrow V$ satisfying $f \in C^{\beta}(Q ; V)$ for all bounded domains $Q \subset \mathbf{R}^{d}$. We call a function $f: \mathbf{R}^{d} \rightarrow \mathbf{R}^{d}$ a $C_{l o c}^{\beta}\left(\mathbf{R}^{d} ; \mathbf{R}^{d}\right)$-diffeomorphism if $f$ is a homeomorphism and both $f$ and its inverse $f^{-1}$ are in $C_{l o c}^{\beta}\left(\mathbf{R}^{d} ; \mathbf{R}^{d}\right)$.

For a Fréchet space $\chi$, we denote by $D([0, T] ; \chi)$ the space of $\chi$-valued càdlàg functions on $[0, T]$. Unless otherwise specified, we endow $D([0, T] ; \chi)$ with the supremum seminorms.

The notation $N=N(\cdot, \cdots, \cdot)$ is used to denote a positive constant depending only on the quantities appearing in the parentheses. In a given context, the same letter is often used to denote different constants depending on the same parameter. If we do not specify to which space the parameters $\omega, t, x, y, z$ and $n$ belong, then we mean $\omega \in \Omega, t \in[0, T], x, y \in \mathbf{R}^{d_{1}}$, $z \in Z^{k}$, and $n \in \mathbf{N}$.

Let $r_{1}(x):=\sqrt{1+|x|^{2}}, \quad x \in \mathbf{R}^{d_{1}}$. Let us introduce some regularity conditions on the coefficients and free terms. We consider these assumptions for $\bar{\beta}>1 \vee \alpha$ and $\tilde{\beta}>\alpha$.

Assumption $2.1(\bar{\beta})$. (1) There is a constant $N_{0}>0$ such that for each $k \in\{1,2\}$ and all $\omega, t \in \Omega \times[0, T]$,

$$
\left|r_{1}^{-1} b_{t}\right|_{0}+\left|\nabla b_{t}\right|_{\bar{\beta}-1}+\left|r_{1}^{-1} \sigma_{t}^{k}\right|_{0}+\left|\nabla \sigma_{t}^{k}\right|_{\bar{\beta}-1} \leq N_{0}
$$

Moreover, for each $k \in\{1,2\}$ and all $(\omega, t, z) \in \Omega \times[0, T] \times\left(D^{k} \cup E^{k}\right)$,

$$
\left|r_{1}^{-1} H_{t}^{k}(z)\right|_{0} \leq K_{t}^{k}(z) \quad \text { and } \quad\left|\nabla H_{t}^{k}(z)\right|_{\bar{\beta}-1} \leq \bar{K}_{t}^{k}(z)
$$

where $K^{k}, \bar{K}^{k}: \Omega \times[0, T] \times\left(D^{k} \cup E^{k}\right) \rightarrow \mathbf{R}_{+}$are $\mathcal{P}_{T} \otimes \mathcal{Z}^{k}$-measurable functions satisfying

$$
K_{t}^{k}(z)+\bar{K}_{t}^{k}(z)+\int_{D^{k}}\left(K_{t}^{k}(z)^{\alpha}+\bar{K}_{t}^{k}(z)^{2}\right) \pi^{k}(d z)+\int_{E^{k}}\left(K_{t}^{k}(z)^{1 \wedge \alpha}+\bar{K}_{t}^{k}(z)\right) \pi^{k}(d z) \leq N_{0},
$$

for all $(\omega, t, z) \in \Omega \times[0, T] \times\left(D^{k} \cup E^{k}\right)$. 
Outline of main results

(2) For each $k \in\{1,2\}$, there is a constant $\eta^{k} \in(0,1)$ such that for all $(\omega, t, x, z) \in\{(\omega, t, x, z) \in$ $\left.\Omega \times[0, T] \times \mathbf{R}^{d_{1}} \times\left(D^{k} \cup E^{k}\right):\left|\nabla H_{t}^{k}(\omega, x, z)\right|>\eta^{k}\right\}$,

$$
\left|\left(I_{d_{1}}+\nabla H_{t}^{k}(x, z)\right)^{-1}\right| \leq N_{0}
$$

Assumption $2.2(\tilde{\beta})$. There is a constant $N_{0}>0$ such that for each $k \in\{1,2\}$ and all $(\omega, t) \in \Omega \times[0, T]$,

$$
\left|c_{t}\right|_{\tilde{\beta}}+\left|v_{t}^{k}\right|_{\tilde{\beta}}+\left|r_{1}^{-\theta} f_{t}\right|_{\tilde{\beta}}+\left|r_{1}^{-\theta} g_{t}\right|_{\tilde{\beta}} \leq N_{0} .
$$

Moreover, for each $k \in\{1,2\}$ and all $(\omega, t, z) \in \Omega \times[0, T] \times\left(D^{k} \cup E^{k}\right)$,

$$
\left|\rho_{t}^{k}(z)\right|_{\tilde{\beta}} \leq l_{t}^{k}(z), \quad\left|r_{1}^{-\theta} h_{t}(z)\right|_{\tilde{\beta}} \leq l_{t}^{k}(z),
$$

where $l^{k}: \Omega \times[0, T] \times Z^{k} \rightarrow \mathbf{R}_{+}$are $\mathcal{P}_{T} \otimes \mathcal{Z}^{k}$-measurable function satisfying

$$
l_{t}^{k}(z)+\int_{D^{k}} l_{t}^{k}(z)^{2} \pi^{k}(d z)+\int_{E^{k}} l_{t}^{k}(z) \pi^{k}(d z) \leq N_{0},
$$

for all $(\omega, t, z) \in \Omega \times[0, T] \times\left(D^{k} \cup E^{k}\right)$.

Remark 2.1. It follows from Lemma 4.10 and Remark 4.11 that if Assumption $2.1(\bar{\beta})$ holds for some $\bar{\beta}>1 \vee \alpha$, then for all $\omega, t$, and $z \in D^{k} \cup E^{k}, x \mapsto \tilde{H}_{t}^{k}(x, z):=x+H_{t}^{k}(x, z)$ is a diffeomorphism.

Let Assumptions 2.1( $\bar{\beta})$ and 2.2( $\tilde{\beta})$ hold for some $\bar{\beta}>1 \vee \alpha$ and $\tilde{\beta}>\alpha$. In our derivation of a solutions of (1.1), we first obtain solutions of equations of a special form. Specifically, consider the system of SIDEs on $[0, T] \times \mathbf{R}^{d_{1}}$ given by

$$
\begin{aligned}
d \hat{u}_{t}^{l}= & \left(\left(\mathcal{L}_{t}^{1 ; l}+\mathcal{L}_{t}^{2 ; l}\right) \hat{u}_{t}+\hat{b}_{t}^{i} \partial_{i} u_{t}^{l}+\hat{c}_{t}^{l \bar{l}} u_{t}^{\bar{l}}+\hat{f}_{t}^{l}\right) d t+\left(\mathcal{N}_{t}^{1 ; l \varrho} \hat{u}_{t}+g_{t}^{l \varrho}\right) d w_{t}^{1 ; \varrho} \\
& +\int_{Z^{1}}\left(\mathcal{I}_{t, z}^{1 ; l} \hat{u}_{t-}+h_{t}^{l}(z)\right)\left[\mathbf{1}_{D^{1}}(z) q^{1}(d t, d z)+\mathbf{1}_{E^{1}}(z) p^{1}(d t, d z)\right], \quad \tau<t \leq T, \\
\hat{u}_{t}^{l}= & \varphi^{l}, \quad t \leq \tau, \quad l \in\left\{1, \ldots, d_{2}\right\},
\end{aligned}
$$

where

$$
\begin{aligned}
\hat{b}_{t}^{i}(x):= & \mathbf{1}_{[1,2]}(\alpha) b_{t}^{i}(x)+\sum_{k=1}^{2} \mathbf{1}_{\{2\}}(\alpha) \sigma_{t}^{k ; j \varrho}(x) \partial_{j} \sigma_{t}^{k ; i \varrho}(x) \\
& +\sum_{k=1}^{2} \mathbf{1}_{(1,2]}(\alpha) \int_{D^{k}}\left(H_{t}^{k ; i}(x, z)-H_{t}^{k ; i}\left(\tilde{H}_{t}^{k ;-1}(x, z), z\right)\right) \pi^{k}(d z), \\
\hat{c}_{t}^{l \bar{l}}(x):= & c_{t}^{l \bar{l}}(x)+\sum_{k=1}^{2} \mathbf{1}_{\{2\}}(\alpha) \sigma_{t}^{k ; j \varrho}(x) \partial_{j} v_{t}^{k ; l \bar{l} \varrho}(x) \\
& +\sum_{k=1}^{2} \int_{D^{k}}\left(\rho_{t}^{k ; l \bar{l}}(x, z)-\rho_{t}^{k ; l \bar{l}}\left(\tilde{H}_{t}^{k ;-1}(x, z), z\right)\right) \pi^{k}(d z), \\
\hat{f}_{t}^{l}(x):= & f_{t}^{l}(x)+\sigma_{t}^{1 ; j \varrho}(x) \partial_{j} g_{t}^{l \varrho}(x)+\int_{D^{1}}\left(h_{t}^{l}(x, z)-h_{t}^{l}\left(\tilde{H}_{t}^{1 ;-1}(x, z), z\right)\right) \pi^{1}(d z) .
\end{aligned}
$$


Let $\left(w_{t}^{2 ; \varrho}\right)_{\varrho \geq 1}, t \geq 0, \varrho \in \mathbf{N}$, be a sequence of independent one-dimensional Wiener processes. Let $p^{2}(d t, d z)$ be a Poisson random measure on $\left([0, \infty) \times Z^{2}, \mathcal{B}\left([0, \infty) \otimes \mathcal{Z}^{2}\right)\right.$ with intensity measure $\pi^{2}(d z) d t$. Extending the probability space if necessary, we take $w^{2}$ and $p^{2}(d t, d z)$ to be independent of $w^{1}$ and $p^{1}(d t, d z)$. Let

$$
\hat{\mathcal{F}}_{t}=\sigma\left(\left(w_{s}^{2}\right)_{\varrho \geq 1}, p^{2}([0, s], \Gamma): s \leq t, \Gamma \in \mathcal{Z}^{2}\right)
$$

and $\tilde{\mathbf{F}}=\left(\tilde{\mathcal{F}}_{t}\right)_{t \leq T}$ be the standard augmentation of $\left(\mathcal{F}_{t} \vee \hat{\mathcal{F}}_{t}\right)_{t \leq T}$. Denote by $q^{2}(d t, d z)=$ $p^{2}(d t, d z)-\pi^{2}(d z) d t$ the compensated Poisson random measure. We associate with the SIDE (2.1), the $\tilde{\mathbf{F}}$-adapted stochastic flow $X_{t}=X_{t}(x)=X_{t}(\tau, x),(t, x) \in[0, T] \times \mathbf{R}^{d_{1}}$, generated by the SDE

$$
\begin{aligned}
d X_{t}= & -\mathbf{1}_{[1,2]}(\alpha) b_{t}\left(X_{t}\right) d t+\sum_{k=1}^{2} \mathbf{1}_{\{2\}}(\alpha) \sigma_{t}^{k ; \varrho}\left(X_{t}\right) d w_{t}^{k ; \varrho} \\
& -\sum_{k=1}^{2} \int_{D^{k}} H_{t}^{k}\left(\tilde{H}_{t}^{k ;-1}\left(X_{t-}, z\right), z\right)\left[p^{k}(d t, d z)-\mathbf{1}_{(1,2]}(\alpha) \pi^{k}(d z) d t\right] \\
& -\sum_{k=1}^{2} \int_{E^{k}} H_{t}^{k}\left(\tilde{H}_{t}^{k ;-1}\left(X_{t-}, z\right), z\right) p^{k}(d t, d z), \quad \tau<t \leq T, \\
X_{t}= & x, \quad t \leq \tau,
\end{aligned}
$$

and the $\tilde{\mathbf{F}}$-adapted random field $\Phi_{t}(x)=\Phi_{t}(\tau, x),(t, x) \in[0, T] \times \mathbf{R}^{d_{1}}$, solving the linear SDE given by

$$
\begin{aligned}
d \Phi_{t}(x)= & \left(c_{t}\left(X_{t}(x)\right) \Phi_{t}(x)+f_{t}\left(X_{t}(x)\right)\right) d t+\sum_{k=1}^{2} v_{t}^{k ; \varrho}\left(X_{t}(x)\right) \Phi_{t}(x) d w_{t}^{k ; \varrho}+g_{t}^{\varrho}\left(X_{t}(x)\right) d w_{t}^{1 ; \varrho} \\
& +\sum_{k=1}^{2} \int_{Z^{k}} \rho_{t}^{k}\left(\tilde{H}_{t}^{k ;-1}\left(X_{t-}(x), z\right), z\right) \Phi_{t-}(x)\left[\mathbf{1}_{D^{k}}(z) q^{k}(d t, d z)+\mathbf{1}_{E^{k}}(z) p^{k}(d t, d z)\right] \\
& +\int_{Z^{1}} h_{t}\left(\tilde{H}_{t}^{1 ;-1}\left(X_{t-}(x), z\right), z\right)\left[\mathbf{1}_{D^{1}}(z) q^{1}(d t, d z)+\mathbf{1}_{E^{1}}(z) p^{1}(d t, d z)\right], \quad \tau<t \leq T, \\
\Phi_{t}(x)= & \varphi(x), \quad t \leq \tau .
\end{aligned}
$$

The coming theorem is our existence, uniqueness, and representation theorem for (2.1). Let us describe our solution class. For each $\beta^{\prime} \in(0, \infty)$, denote by $\widetilde{\leftarrow}^{\beta^{\prime}}\left(\mathbf{R}^{d_{1}} ; \mathbf{R}^{d_{2}}\right)$ the linear space of all $\mathbf{F}$-adapted random fields $v=v_{t}(x)$ such that $\mathbf{P}$-a.s.

$$
\mathbf{1}_{\left[\tau_{n}, \tau_{n+1}\right)} r_{1}^{-\lambda_{n}} v \in D\left([0, T] ; C^{\beta^{\prime}}\left(\mathbf{R}^{d_{1}}, \mathbf{R}^{d_{2}}\right)\right),
$$

where $\left(\tau_{n}\right)_{n \geq 0}$ is an increasing sequence of $\mathbf{F}$-stopping times with $\tau_{0}=0$ and $\tau_{n}=T$ for sufficiently large $n$, and where for each $n, \lambda_{n}$ is a positive $\mathcal{F}_{\tau_{n}}$-measurable random variable. 
Theorem 2.2. Let Assumptions 2.1( $\bar{\beta})$ and 2.2( $\tilde{\beta})$ hold for some $\bar{\beta}>1 \vee \alpha$ and $\tilde{\beta}>\alpha$. For each stopping time $\tau \leq T$ and $\mathcal{F}_{\tau} \otimes \mathcal{B}\left(\mathbf{R}^{d_{1}}\right)$-measurable random field $\varphi$ such that for some $\beta^{\prime} \in(\alpha, \bar{\beta} \wedge \tilde{\beta})$ and $\theta^{\prime} \geq 0$, P-a.s. $r_{1}^{-\theta^{\prime}} \varphi \in C^{\beta^{\prime}}\left(\mathbf{R}^{d_{1}} ; \mathbf{R}^{d_{2}}\right)$, there exists a unique solution $\hat{u}=\hat{u}(\tau)$ of $(2.1)$ in $\widetilde{C}^{\beta^{\prime}}\left(\mathbf{R}^{d_{1}} ; \mathbf{R}^{d_{2}}\right)$ and for all $(t, x) \in[0, T] \times \mathbf{R}^{d_{1}}, \mathbf{P}$-a.s.

$$
\hat{u}_{t}(\tau, x)=\mathbf{E}\left[\Phi_{t}\left(\tau, X_{t}^{-1}(\tau, x)\right) \mid \mathcal{F}_{t}\right]
$$

Moreover, for each $\epsilon>0$ and $p \geq 2$,

$$
\mathbf{E}\left[\sup _{t \leq T}\left|r_{1}^{-\theta \vee \theta^{\prime}-\epsilon} \hat{u}_{t}(\tau)\right|_{\beta^{\prime}}^{p} \mid \mathcal{F}_{\tau}\right] \leq N\left(\left|r_{1}^{-\theta^{\prime}} \varphi\right|_{\beta^{\prime}}^{p}+1\right),
$$

for a constant $N=N\left(d_{1}, d_{2}, p, N_{0}, T, \beta^{\prime}, \eta^{1}, \eta^{2}, \epsilon, \theta, \theta^{\prime}\right)$.

Using Itô's formula it is easy to check that if $m=1$ and

$$
g_{t}(x)=0, \quad h_{t}(x)=0, \quad \text { and } \quad \rho_{t}^{k}(x, z) \geq-1,
$$

for all $(\omega, t, x, z) \in \Omega \times[[\tau, T]] \times \mathbf{R}^{d_{1}} \times\left(D^{k} \cup E^{k}\right), k \in\{1,2\}$, then

$$
\Phi_{t}(x)=\Psi_{t}(x) \phi(x)+\Psi_{t}(x) \int_{] \tau, \tau \vee t]} \Psi_{s}^{-1}(x) f_{s}\left(X_{s}(x)\right) d s,
$$

where $\mathbf{P}$-a.s. for all $t$ and $x$,

$$
\begin{aligned}
\Psi_{t}(x)= & e^{\int_{[\tau, \tau \vee t]}\left(c_{s}\left(X_{s}(x)\right)-\sum_{k=1}^{2} \frac{1}{2} v_{s}^{k ; \varrho}\left(X_{s}(x)\right) v_{s}^{k ; \varrho}\left(X_{s}(x)\right)\right) d s+\sum_{k=1}^{2} \int_{] \tau, \tau \vee t]} v_{s}^{k ; \varrho}\left(X_{s}(x)\right) d w_{s}^{k ; \varrho}} \\
& \cdot e^{-\sum_{k=1}^{2} \int_{[\tau, \tau \vee t]} \int_{D^{k}}\left(\ln \left(1+\rho_{s}^{k}\left(\tilde{H}_{s}^{k ;-1}\left(X_{s-}(x), z\right), z\right)\right)-\rho_{s}^{k}\left(\tilde{H}_{s}^{k ;-1}\left(X_{s-}(x), z\right), z\right)\right) \pi^{k}(d z) d s} \\
& \cdot e^{\sum_{k=1}^{2} \int_{], \tau \vee t]} \int_{Z^{k}} \ln \left(1+\rho_{s}^{k}\left(\tilde{H}_{s}^{k ;-1}\left(X_{s-}(x), z\right), z\right)\right)\left[\mathbf{1}_{D^{k}}(z) q^{k}(d s, d z)+\mathbf{1}_{E^{k}}(z) p^{k}(d s, d z)\right]} .
\end{aligned}
$$

The following corollary then follows directly from (2.3) and the (2.5).

Corollary 2.3. Let $m=1$ and assume that

$$
g_{t}(x)=0, \quad h_{t}(x, z)=0, \quad \rho_{t}^{k}(x, z) \geq-1, \quad \forall(\omega, t, x, z) \in[[\tau, T]] \times \mathbf{R}^{d_{1}} \times\left(D^{k} \cup E^{k}\right), k \in\{1,2\} .
$$

Moreover, let Assumptions 2.1 $(\bar{\beta})$ and 2.2( $\tilde{\beta})$ hold for some $\bar{\beta}>1 \vee \alpha$ and $\tilde{\beta}>\alpha$. Let $\tau \leq T$ be stopping time and $\varphi$ be $a \mathcal{F}_{\tau} \otimes \mathcal{B}\left(\mathbf{R}^{d_{1}}\right)$-measurable random field such that for some $\beta^{\prime} \in(\alpha, \bar{\beta} \wedge \tilde{\beta})$ and $\theta^{\prime} \geq 0$, P-a.s. $r_{1}^{-\theta^{\prime}} \varphi \in C^{\beta^{\prime}}\left(\mathbf{R}^{d_{1}} ; \mathbf{R}^{d_{2}}\right)$.

(1) If for all $(\omega, t, x) \in[[\tau, T]] \times \mathbf{R}^{d_{1}}, f_{t}(x) \geq 0$ and $\varphi(x) \geq 0$, then the solution $\hat{u}$ of (1.1) satisfies $\hat{u}_{t}(x) \geq 0, \mathbf{P}$-a.s. for all $(t, x) \in[0, T] \times \mathbf{R}^{d_{1}}$.

(2) If for all $(\omega, t, x, z) \in[[\tau, T]] \times \mathbf{R}^{d_{1}} \times\left(D^{k} \cup E^{k}\right), k \in\{1,2\}, v_{t}^{k}(x)=0, f_{t}(x) \leq 0, c_{t}(x) \leq 0$, $\varphi(x) \leq 1$, and $\rho_{t}^{k}(x, z) \leq 0$, then the solution $\hat{u}$ of (1.1) satisfies $\hat{u}_{t}(x) \leq 1$, P-a.s. for all $(t, x) \in[0, T] \times \mathbf{R}^{d_{1}}$. 
Remark 2.4. Since $\mathcal{L}^{2}$ can be the zero operator, both Theorem 2.2 and Corollary 2.3 apply to fully degenerate equations and partial differential equations with random coefficients.

Now, let us discuss our existence and uniqueness theorem for (1.1). We construct the solution of $u=u(\tau)$ of (1.1) by interlacing the solutions of (2.1) along a sequence of large jump moments (see Section 3.5). By using an interlacing procedure we are also able to drop the condition of boundedness of $\left(I+\nabla H_{t}^{1}(x, z)\right)^{-1}$ on the set $(\omega, t, x, z) \in\{(\omega, t, x, z) \in$ $\left.\Omega \times[0, T] \times \mathbf{R}^{d_{1}} \times\left(D^{1} \cup E^{1}\right):\left|\nabla H_{t}^{1}(\omega, x, z)\right|>\eta^{k}\right\}$. Also, in order to remove the terms in $\hat{b}$, $\hat{c}$, and $\hat{f}$ that appear in (2.1), but not in (1.1), we subtract terms from the relevant coefficients in the flow and the transformation. However, in order to do this, we need to impose stronger regularity assumptions on some of the coefficients and free terms. We will introduce the parameters $\mu^{1}, \mu^{2}, \delta^{1}, \delta^{2} \in\left[0, \frac{\alpha}{2}\right]$, which essentially allows one to trade-off integrability in $z$ and regularity in $x$ of the coefficients $H_{t}^{k}(x, z), \rho_{t}^{k}(x, z), h_{t}^{k}(x, z)$. It is worth mentioning that the removal of terms and the interlacing procedure are independent of each other and that it is due only to the weak assumptions on $H^{1}$ and $\rho^{1}$ on the set $V^{1}$ that we do not have moment estimates and a simple representation property like (2.4) for the solution of (1.1). Nevertheless, there is a representation of sorts and we refer the reader to the proof of the coming theorem for an explicit construction of the solution.

We introduce the following assumption for $\bar{\beta}>1 \vee \alpha, \tilde{\beta}>\alpha$, and $\delta^{1}, \delta^{2}, \mu^{1}, \mu^{2} \in\left[0, \frac{\alpha}{2}\right]$.

Assumption $2.3\left(\bar{\beta}, \mu^{1}, \mu^{2}, \delta^{1}, \delta^{2}\right)$. (1) There is a constant $N_{0}>0$ such that for each $k \in$ $\{1,2\}$ and all $(\omega, t) \in \Omega \times[0, T]$,

$$
\left|r_{1}^{-1} b_{t}\right|_{0}+\left|\nabla b_{t}\right|_{\bar{\beta}-1}+\left|\sigma_{t}^{k}\right|_{\bar{\beta}+1} \leq N_{0} .
$$

(2) For each $k \in\{1,2\}$ and all $(\omega, t) \in \Omega \times[0, T]$,

$$
\begin{gathered}
\left|H_{t}^{k}(z)\right|_{0} \leq K_{t}^{k}(z), \quad\left|\nabla H_{t}^{k}(z)\right|_{\bar{\beta}-1}, \forall z \in D^{k}, \\
\left|r_{1}^{-1} H_{t}^{k}(z)\right|_{0} \leq K_{t}^{k}(z), \quad\left|\nabla H_{t}^{k}(z)\right|_{\bar{\beta}-1} \leq \bar{K}_{t}^{k}(z), \quad \forall z \in E^{k}, \\
\left|\rho^{k}(t, z)\right|_{\bar{\beta}} \leq l_{t}^{k}(z), \quad \forall z \in D^{k}, \quad\left|r_{1}^{-\theta} h_{t}(z)\right|_{\bar{\beta}} \leq l_{t}^{1}(z), \quad \forall z \in D^{1},
\end{gathered}
$$

where $K^{k}, \bar{K}^{k}, l^{k}: \Omega \times[0, T] \times\left(D^{k} \cup E^{k}\right) \rightarrow \mathbf{R}_{+}$are $\mathcal{P}_{T} \otimes \mathcal{Z}^{k}$-measurable functions satisfying for all $(\omega, t, z) \in \Omega \times[0, T] \times\left(D^{k} \cup E^{k}\right)$,

$$
K_{t}^{k}(z)+\bar{K}_{t}^{k}(z)+l_{t}^{k}(z) \leq N_{0}
$$

and

$$
\int_{D^{k}}\left(K_{t}^{k}(z)^{\alpha}+\bar{K}_{t}^{k}(z)^{2}+l_{t}^{k}(z)^{2}\right) \pi^{k}(d z)+\int_{E^{k}}\left(K_{t}^{k}(z)^{1 \wedge \alpha}+\bar{K}_{t}^{k}(z)\right) \pi^{k}(d z) \leq N_{0}
$$


(3) For each $k \in\{1,2\}$ and all $(\omega, t) \in \Omega \times[0, T]$,

$$
\begin{gathered}
\left|v_{t}^{k}\right|_{\bar{\beta}+1} \leq N_{0}, \text { if } \sigma_{t}^{k} \neq 0, \quad \mid g_{t \mid \bar{\beta}+1} \leq N_{0}, \text { if } \sigma_{t}^{1} \neq 0, \\
\left.\sum_{|\gamma|=[\bar{\beta}]^{-}} \mid \partial^{\gamma} H_{t}^{k}(z)\right)\left.\right|_{\{\bar{\beta}\}^{+}+\delta^{k}} \leq \tilde{K}_{t}^{k}(z), \forall z \in D^{k}, \text { if }\{\bar{\beta}\}^{+}+\delta^{k} \leq 1, \\
\left.\sum_{|\gamma|=[\bar{\beta}]^{-}}\left|\nabla \partial^{\gamma} H_{t}^{k}(z)\right|_{0} \leq \bar{K}_{t}^{k}(z), \sum_{|\gamma|=[\bar{\beta}]^{-}} \mid \nabla \partial^{\gamma} H_{t}^{k}(z)\right)\left.\right|_{\{\bar{\beta}\}^{+}+\delta^{k}-1} \leq \tilde{K}_{t}^{k}(z), \forall z \in D^{k}, \text { if }\{\bar{\beta}\}^{+}+\delta^{k}>1, \\
\left.\sum_{|\gamma|=[\bar{\beta}]^{-}} \mid \partial^{\gamma} \rho_{t}^{k}(z)\right)\left.\right|_{\{\bar{\beta}\}^{+}+\mu^{k}} \leq \tilde{l}_{t}^{k}(z), \forall z \in D^{k}, \text { if }\{\bar{\beta}\}^{+}+\mu^{k} \leq 1, \\
\left.\sum_{|\gamma|=[\bar{\beta}]^{-}}\left|\nabla \partial^{\gamma} \rho_{t}^{k}(z)\right|_{0} \leq l_{t}^{k}(z), \quad \sum_{|\gamma|=[\bar{\beta}]^{-}} \mid \nabla \partial^{\gamma} \rho_{t}^{k}(z)\right)\left.\right|_{\{\bar{\beta}\}^{+}+\mu^{k}-1} \leq \tilde{l}_{t}^{k}(z), \forall z \in D^{k}, \text { if }\{\bar{\beta}\}^{+}+\mu^{k}>1, \\
\left.\sum_{|\gamma|=[\bar{\beta}]^{-}}\left|\nabla \partial^{\gamma} h_{t}^{1}(z)\right|_{0} \leq l_{t}^{1}(z), \sum_{|\gamma|=[\bar{\beta}]^{-}} \mid \nabla \partial^{\gamma} h_{t}(z)\right)\left.\right|_{\{\bar{\beta}\}^{+}+\mu^{1}-1} \leq \tilde{l}_{t}^{1}(z), \forall z \in D^{1}, \text { if }\{\bar{\beta}\}^{+}+\mu^{1}>1,
\end{gathered}
$$

where $\tilde{K}^{k}, \tilde{l}^{k}: \Omega \times[0, T] \times D^{k} \rightarrow \mathbf{R}_{+}$are $\mathcal{P}_{T} \otimes \mathcal{Z}^{k}$-measurable functions satisfying for all $(\omega, t, z) \in \Omega \times[0, T] \times D^{k}$,

$$
\tilde{K}_{t}^{k}(z)+\tilde{l}_{t}^{k}(z)+\int_{D^{k}}\left(\tilde{K}_{t}^{k}(z)^{\frac{\alpha}{\alpha-\delta^{k}}} \mathbf{1}_{\left[0, \frac{\alpha}{2}\right]}\left(\delta^{k}\right)+\tilde{K}_{t}^{k}(z)^{2}+\tilde{l}_{t}^{k}(z)^{\frac{\alpha}{\alpha-\mu^{k}}} \mathbf{1}_{\left[0, \frac{\alpha}{2}\right]}\left(\mu^{k}\right)+\tilde{l}_{t}^{k}(z)^{2}\right) \pi^{k}(d z) \leq N_{0} .
$$

(4) There is a constant $\eta^{2} \in(0,1)$ such that for all $(\omega, t, x, z) \in\{(\omega, t, x, z) \in \Omega \times[0, T] \times$ $\left.\mathbf{R}^{d_{1}} \times Z^{2}:\left|\nabla H_{t}^{2}(\omega, x, z)\right|>\eta^{2}\right\}$

$$
\left|\left(I_{d_{1}}+\nabla H_{t}^{2}(x, z)\right)^{-1}\right| \leq N_{0}
$$

Assumption $2.4(\tilde{\beta})$. (1) There is a constant $N_{0}>0$ such that for each $k \in\{1,2\}$ and all $(\omega, t) \in \Omega \times[0, T]$,

$$
\begin{gathered}
\left|c_{t}\right|_{\tilde{\beta}}+\left|r_{1}^{-\theta} f_{t}\right|_{\tilde{\beta}} \leq N_{0}, \\
\left|v_{t}^{k}\right|_{\tilde{\beta}} \leq N_{0}, \text { if } \sigma_{t}^{k}=0, \quad\left|g_{t}\right|_{\tilde{\beta}} \leq N_{0}, \text { if } \sigma_{t}^{1}=0, \\
\left|\rho^{k}(t, z)\right|_{\tilde{\beta}} \leq l_{t}^{k}(z), \quad \forall z \in E^{k}, \quad\left|r_{1}^{-\theta} h_{t}(z)\right|_{\tilde{\beta}} \leq l_{t}^{1}(z), \forall z \in E^{1},
\end{gathered}
$$

where for all $(\omega, t) \in \Omega \times[0, T], \int_{E^{k}} l_{t}^{k}(z) \pi^{k}(d z) \leq N_{0}$.

(2) There exist processes $\xi, \zeta: \Omega \times[0, T] \times V^{1} \rightarrow \mathbf{R}_{+}$that are $\mathcal{P}_{T} \otimes \mathcal{Z}^{1}$ measurable satisfying

$$
\left|r_{1}^{-\xi_{t}(z)} H_{t}^{1}(z)\right|_{\tilde{\beta} \vee 1}+\left|r_{1}^{-\xi_{t}(z)} \rho_{t}^{1}(z)\right|_{\tilde{\beta}}+\left|r_{1}^{-\xi_{t}(z)} h_{t}(z)\right|_{\tilde{\beta}} \leq \zeta_{t}(z),
$$

for all $(\omega, t, z) \in \Omega \times[0, T] \times V^{1}$.

We now state our existence and uniqueness theorem for (1.1).

Theorem 2.5. Let Assumptions $2.3\left(\bar{\beta}, \delta^{1}, \delta^{2}, \mu^{1}, \mu^{2}\right)$ and $2.4(\tilde{\beta})$ hold for some $\bar{\beta}>1 \vee \alpha$, $\tilde{\beta}>\alpha$, and $\delta^{1}, \delta^{2}, \mu^{1}, \mu^{2} \in\left[0, \frac{\alpha}{2}\right]$. For each stopping time $\tau \leq T$ and $\mathcal{F}_{\tau} \otimes \mathcal{B}\left(\mathbf{R}^{d_{1}}\right)$-measurable random field $\varphi$ such that for some $\beta^{\prime} \in(\alpha, \bar{\beta} \wedge \tilde{\beta})$ and $\theta^{\prime} \geq 0$, P-a.s. $r_{1}^{-\theta^{\prime}} \varphi \in C^{\beta^{\prime}}\left(\mathbf{R}^{d_{1}} ; \mathbf{R}^{d_{2}}\right)$, there exists a unique solution $u=u(\tau)$ of $(1.1)$ in $\mathfrak{C}^{\beta^{\prime}}\left(\mathbf{R}^{d_{1}} ; \mathbf{R}^{d_{2}}\right)$. 


\section{Proof of main theorems}

We will first prove uniqueness of the solution of (2.1) in the class $\leftarrow^{\beta^{\prime}}\left(\mathbf{R}^{d_{1}} ; \mathbf{R}^{d_{2}}\right)$. The existence part of the proof of Theorem 2.2 is divided into a series of steps. In the first step, by appealing to the representation theorem we derived for solutions of continuous SPDEs in Theorem 2.4 in [LM14b], we use an interlacing procedure and the strong limit theorem given in Theorem 2.3 in [LM14b] to show that the space inverse of the flow generated by a jump SDE (i.e. the SDE (2.2) without the uncorrelated noise) solves a degenerate linear SIDE. Then we linearly transform the inverse flow of a jump SDE to obtain solutions of degenerate linear SIDEs with free and zero-order terms and an initial condition. In the last step of the proof of Theorem 2.2, we introduce an independent Wiener process and Poisson random measure as explained above, apply the results we know for fully degenerate equations, and then take the optional projection of the equation. In the last section, Section 3.4, we prove Theorem 2.5 using an interlacing procedure and removing the extra terms in $\hat{b}, \hat{c}$ and $\hat{f}$. The uniqueness of the solution $u$ of (1.1) follows directly from our construction.

\subsection{Proof of uniqueness for Theorem 2.2}

Proof of Uniqueness for Theorem 2.2. Fix a stopping time $\tau \leq T$ and $\mathcal{F}_{\tau} \otimes \mathcal{B}\left(\mathbf{R}^{d_{1}}\right)$-measurable random field $\varphi$ such that for some $\beta^{\prime} \in(\alpha, \bar{\beta} \wedge \tilde{\beta})$ and $\theta^{\prime} \geq 0$, P-a.s. $r_{1}^{-\theta^{\prime}} \varphi \in C^{\beta^{\prime}}\left(\mathbf{R}^{d_{1}} ; \mathbf{R}^{d_{2}}\right)$. In this section we will drop the dependence of processes $t, x$, and $z$ when we feel it will not obscure the argument. Let $\hat{u}^{1}(\tau)$ and $\hat{u}^{2}(\tau)$ be solutions of $(2.1)$ in $\widetilde{\sigma}^{\beta^{\prime}}$. It follows that $v:=\hat{u}^{1}(\tau)-\hat{u}^{2}(\tau)$ solves

$$
\begin{aligned}
d v_{t}^{l}= & {\left[\left(\mathcal{L}_{t}^{1 ; l}+\mathcal{L}_{t}^{2 ; l}\right) v_{t}+\hat{b}_{t}^{i} \partial_{i} v_{t}^{l}+\hat{c}_{t}^{l \bar{l}} v_{t}^{\bar{l}}\right] d t+\mathcal{N}_{t}^{1 ; \varrho} v_{t}^{l} d w_{t}^{1 ; \varrho} } \\
& +\int_{Z^{1}} \mathcal{I}_{t, z}^{1 ; l} v_{t-}\left[\mathbf{1}_{D^{1}}(z) q^{1}(d t, d z)+\mathbf{1}_{E^{1}}(z) p^{1}(d t, d z)\right], \quad \tau<t \leq T, \\
v_{t}^{l}= & 0, \quad t \leq \tau, \quad l \in\left\{1, \ldots, d_{2}\right\},
\end{aligned}
$$

and $\mathbf{P}$-a.s.

$$
\mathbf{1}_{\left[\tau_{n}, \tau_{n+1}\right)} r_{1}^{-\lambda_{n}} v \in D\left([0, T] ; C^{\beta^{\prime}}\left(\mathbf{R}^{d_{1}}, \mathbf{R}^{d_{2}}\right)\right),
$$

where $\left(\tau_{n}\right)_{n \geq 0}$ is an increasing sequence of $\mathbf{F}$-stopping times with $\tau_{0}=0$ and $\tau_{n}=T$ for sufficiently large $n$, and where for each $n, \lambda_{n}$ is a positive $\mathcal{F}_{\tau_{n}}$-measurable random variable. Clearly it suffices to take $\tau_{1}=\tau$ and $\lambda_{0}=0$. Thus, $v_{t}(x)=0$ for all $(\omega, t) \in\left[\left[\tau_{0}, \tau_{1}\right)\right)$. Assume that for some $n, \mathbf{P}$-a.s. for all $t$ and $x, v_{t \wedge \tau_{n}}(x)=0$. We will show that $\mathbf{P}$-a.s. for all $t$ and $x$, $\tilde{v}_{t}(x):=v_{\left(\tau_{n} \vee t\right) \wedge \tau_{n+1}}(x)=0$. Applying Itô's formula, for each $x$, P-a.s. for all $t$, we find

$$
\begin{aligned}
d\left|\tilde{v}_{t}\right|^{2}= & \left(2 \tilde{v}_{t}^{l} \mathfrak{Q}_{t}^{1 ; l} \tilde{v}_{t}+\left|\mathcal{N}_{t}^{1} \tilde{v}_{t}\right|^{2}+2 \tilde{v}_{t}^{l} b_{t}^{i} \partial_{i} \tilde{v}_{t}^{l}+2 \tilde{v}_{t}^{l} c_{t}^{l \bar{l}} \tilde{v}_{t}^{\bar{l}}\right) d t \\
& +\left(2 \tilde{v}_{t}^{l} \mathfrak{\jmath}_{t, z}^{1 ; l} \tilde{v}_{t}+\int_{D^{1} \cup E^{1}}\left|\mathcal{I}_{t, z}^{1 ; l} \tilde{v}_{t}\right|^{2} \pi^{1}(d z)\right) d t \\
& +\left(2 v_{t}^{l} \mathfrak{Q}_{t}^{2 ; l} \tilde{v}_{t}+2 \tilde{v}_{t}^{l} \mathfrak{\Im}_{t, z}^{2 ; l} \tilde{v}_{t}\right) d t+2 v_{t}^{l} \mathcal{N}_{t}^{1 ; \varrho} \tilde{v}_{t}^{l} d w_{t}^{1 ; \varrho}
\end{aligned}
$$




$$
\begin{aligned}
& +\int_{Z^{1}}\left(2 \tilde{v}_{t-}^{l} \mathcal{I}_{t, z}^{1 ; l} \tilde{v}_{t-}+\left|\mathcal{I}_{t, z}^{1 ; l} \tilde{v}_{t-}\right|^{2}\right) q^{1}(d t, d z), \quad \tau_{n}<t \leq \tau_{n+1}, \\
\left|\tilde{v}_{t}\right|^{2}= & 0, \quad t \leq \tau_{n}, \quad l \in\left\{1, \ldots, d_{2}\right\},
\end{aligned}
$$

where for $\phi \in C_{c}^{\infty}\left(\mathbf{R}^{d_{1}} ; \mathbf{R}^{d_{2}}\right), k \in\{1,2\}$, and $l \in\left\{1, \ldots, d_{2}\right\}$,

$$
\mathfrak{2}^{k ; l} \phi:=\frac{1}{2} \sigma^{k ; \varrho \varrho} \sigma^{k ; j \varrho} \partial_{i j} \phi^{l}+\sigma^{k ; j \varrho} \partial_{j} \sigma^{k ; \varrho \varrho} \partial_{i} \phi^{l}+\sigma^{k ; \varrho \varrho} v^{k ; l \bar{l} \varrho} \partial_{i} \phi^{\bar{l}}+\sigma^{k ; \varrho} \partial_{j} a^{k ; \bar{l} \varrho} \phi^{\bar{l}}
$$

and

$$
\begin{aligned}
\mathfrak{J}^{k ; l} \phi:= & \int_{D^{k}}\left(\rho^{k ; \bar{l}} \phi^{\bar{l}}\left(\tilde{H}^{k}\right)-\rho^{k ; \bar{l}}\left(\tilde{H}^{k ;-1}\right) \phi^{\bar{l}}\right) \pi^{k}(d z) \\
& +\int_{D^{k}}\left(\phi^{l}\left(\tilde{H}^{k}\right)-\phi^{l}+\mathbf{1}_{(1,2]}(\alpha) F^{k ; i} \partial_{i} \phi^{l}\right) \pi^{k}(d z) \\
& +\int_{E^{k}}\left(\left(I_{d_{2}}^{l \bar{l}}+\rho^{k ; l \bar{l}}\right) \phi^{\bar{l}}\left(\tilde{H}^{k}\right)-\phi^{l}\right) \pi^{k}(d z) .
\end{aligned}
$$

For each $\omega$ and $t$, let

$$
Q_{t}=\int_{\mathbf{R}^{d^{1}}}\left|\tilde{v}_{t}(x)\right|^{2} r_{1}^{-\lambda}(x) d x,
$$

where $\lambda=\lambda_{n}+\left(d^{\prime}+2\right) / 2$ and $d^{\prime}>d_{1}$. Note that

$$
\mathbf{E} Q_{t} \leq \int_{\mathbf{R}^{d^{1}}} r_{1}^{-d^{\prime}}(x) d x \mathbf{E}\left|r_{1}^{-\lambda_{n}} \tilde{v}_{t}\right|_{0}<\infty .
$$

It suffices to show that $\sup _{t \leq T} \mathbf{E} Q_{t}=0$. To this end, we will multiply the equation (3.1) by the weight $r_{1}^{-2 \lambda}=r_{1}^{-2 \lambda_{n}+1} r_{1}^{-d^{\prime}}$, integrate in $x$, and change the order of the integrals in time and space. Thus, we must verify the assumptions of stochastic Fubini theorem hold (see Corollary 4.13 and Remark 4.14 as well) with the finite measure $\mu(d x)=r_{1}^{-d^{\prime}}(x) d x$ on $\mathbf{R}^{d_{1}}$. Since $b$ and $\sigma^{k}$ have linear growth an $v^{k}$ and $c$ are bounded, owing to Lemma 4.6, we easily obtain that there is a constant $N=N\left(d_{1}, d_{2}, N_{0}, \lambda_{n}\right)$ such that $\mathbf{P}$-a.s for all $t$,

$$
\begin{gathered}
\int_{\mathbf{R}^{d_{1}}}\left(\sum_{k=1}^{2} 2\left|r_{1}^{-\lambda_{n}} \tilde{v}\right|\left|r_{1}^{-\lambda_{n}-2} \mathfrak{Q}^{k} \tilde{v}\right|+\left|r_{1}^{\lambda_{n}-1} \mathcal{N}^{1} \tilde{v}\right|^{2}\right) r_{1}^{-d^{\prime}} d x \leq N \sup _{t \leq T}\left|r_{1}^{-\lambda_{n}} \tilde{v}\right|_{\beta^{\prime}}^{2}, \\
\int_{\mathbf{R}^{d_{1}}} 4\left|r_{1}^{-\lambda_{n}} \tilde{v}\right|^{2}\left|r_{1}^{-\lambda_{n}-1} \mathcal{N}^{1} \tilde{v}\right|^{2} r_{1}^{-d^{\prime}} d x \leq N \sup _{t \leq T}\left|r_{1}^{-\lambda_{n}} \tilde{v}_{t}\right|_{\beta^{\prime}}^{4},
\end{gathered}
$$

and

$$
\int_{\mathbf{R}^{d_{1}}}\left(2\left|r_{1}^{-\lambda_{n}} \tilde{v}\right| r_{1}^{-\lambda_{n}-1} b \partial_{i} \tilde{v}|+2| r_{1}^{-\lambda_{n}} \tilde{v} \| r_{1}^{-\lambda_{n}} c \tilde{v} \mid\right) r_{1}^{-d^{\prime}} d x \leq N \sup _{t \leq T}\left|r_{1}^{-\lambda_{n}} \tilde{v}_{t}\right|_{\beta^{\prime}}^{2}
$$


For all $\phi \in C_{l o c}^{\alpha}\left(\mathbf{R}^{d_{1}} ; \mathbf{R}^{d_{2}}\right)$ and all $k, \omega, t, x, p$, and $z$

$$
\begin{gathered}
r_{1}^{-p}\left(\phi\left(\tilde{H}^{k}\right)-\phi+\mathbf{1}_{(1,2]}(\alpha) F^{k ; i} \partial_{i} \phi\right) \\
=\bar{\phi}\left(\tilde{H}^{k}\right)-\bar{\phi}-\mathbf{1}_{(1,2]}(\alpha) H^{k ; i} \partial_{i} \bar{\phi}+\mathbf{1}_{(1,2]}(\alpha)\left(H^{k ; i}+F^{k ; i}\right) \partial_{i} \bar{\phi} \\
+p \mathbf{1}_{(1,2]}(\alpha)\left(H^{k ; i}+F^{k ; i}\right) r_{1}^{-2} x^{i} \bar{\phi}+\left(\frac{r_{1}^{p}\left(\tilde{H}^{k}\right)}{r_{1}^{p}}-1\right)\left(\bar{\phi}\left(\tilde{H}^{k}\right)-\mathbf{1}_{(1,2]}(\alpha) \bar{\phi}\right) \\
+\mathbf{1}_{(1,2]}(\alpha)\left(\frac{r_{1}^{p}\left(\tilde{H}^{k}\right)}{r_{1}^{p}}-1+p H^{k ; i} r_{1}^{-2} x^{i}\right) \bar{\phi}
\end{gathered}
$$

where $\bar{\phi}:=r^{-p} \phi$. By Taylor's formula, for all $\phi \in C^{\alpha}\left(\mathbf{R}^{d_{1}} ; \mathbf{R}^{d_{2}}\right)$ and all $k, \omega, t, x$, and $z$, we have

$$
\left|\phi\left(\tilde{H}^{k}\right)-\phi-\mathbf{1}_{(1,2]}(\alpha) H^{k ; i} \partial_{i} \phi\right| \leq r_{1}^{\alpha}|\phi|_{\alpha}\left|r_{1}^{-1} H\right|_{0}^{\alpha} .
$$

Combining (3.2), (3.3), and the estimates given in Lemma 4.10 (1), for all $k, \omega, t, x$ and $z$, we obtain

$$
r_{1}^{-\alpha}\left|\rho^{k}\left(\tilde{H}^{k ;-1}\right)-\rho^{k}\right| \leq N|\rho|_{\alpha \wedge 1}\left|r_{1}^{-1} H^{k}\right|_{0}^{\alpha \wedge 1}
$$

and

$$
\begin{gathered}
r_{1}^{-\lambda_{n}-\alpha}\left|\tilde{v}\left(\tilde{H}^{k}\right)-\tilde{v}+\mathbf{1}_{(1,2]}(\alpha) F^{k ; i} \partial_{i} \tilde{v}\right| \\
\leq N\left|r_{1}^{-\lambda_{n}} \tilde{v}\right|_{\alpha}\left(\left|r_{1}^{-1} H^{k}\right|_{0}^{\alpha}+\left|r_{1}^{-1} H\right|_{0}\left[H^{k}\right]_{1}+\left|r_{1}^{-1} H\right|_{0}^{[\alpha]^{-}+1}+[H]_{1}^{[\alpha]^{-}+1}\right),
\end{gathered}
$$

for some constant $N=N\left(d_{1}, \lambda_{n}, N_{0}, \eta^{1}, \eta^{2}\right)$. Therefore, $\mathbf{P}$-a.s for all $t$,

$$
\int_{\mathbf{R}^{d_{1}}}\left(\sum_{k=1}^{2} 2\left|r_{1}^{-\lambda_{n}} \tilde{v} \| r_{1}^{-\lambda_{n}-2} \mathfrak{J}^{k} \tilde{v}\right|+\int_{D^{1} \cup E^{1}}\left|r_{1}^{-\lambda-1} \mathcal{I}_{z} \tilde{v}\right|^{2} \pi^{1}(d z)\right) r_{1}^{-d^{\prime}} d x \leq N \sup _{t \leq T}\left|r_{1}^{-\lambda_{n}} \tilde{v}\right|_{\beta^{\prime}}^{2},
$$

and

$$
\int_{\mathbf{R}^{d_{1}}}\left(2\left|r_{1}^{-\lambda_{n}} \tilde{v}\right|\left|r_{1}^{-\lambda_{n}-2} \mathcal{I}_{z}^{k} \tilde{v}\right|+\left|r_{1}^{-\lambda_{n}-1} \mathcal{I}_{z} \tilde{v}\right|^{2}\right)^{2} r_{1}^{-d^{\prime}} d x \leq N \sup _{t \leq T}\left|r_{1}^{-\lambda_{n}} \tilde{v}_{t}\right|_{\beta^{\prime}}^{4}
$$

for some constant $N=N\left(d_{1}, d_{2}, \lambda_{n}, N_{0}, \eta^{1}, \eta^{2}\right)$.

Let $L^{2}\left(\mathbf{R}^{d_{1}} ; \mathbf{R}^{d_{2}}\right)$ be the space of square-integrable functions $f: \mathbf{R}^{d_{1}} \rightarrow \mathbf{R}^{d_{2}}$ with norm $\|\cdot\|_{0}$ and inner product $(\cdot, \cdot)_{0}$. Moreover, let $L^{2}\left(\mathbf{R}^{d_{1}} ; \ell_{2}\left(\mathbf{R}^{d_{2}}\right)\right)$ be the space of square-integrable functions $f: \mathbf{R}^{d_{1}} \rightarrow \ell_{2}\left(\mathbf{R}^{d_{2}}\right)$ with norm $\|\cdot\|_{0}$. With the help of the above estimates and Corollary 4.13, denoting $\bar{v}=r^{-\lambda} \tilde{v}, \mathbf{P}$-a.s. for all $t$, we have

$$
\begin{aligned}
d\left\|\bar{v}_{t}\right\|_{0}^{2}= & \left(2\left(\bar{v}_{t}^{l}, \overline{\mathfrak{I}}_{t}^{1} \bar{v}_{t}\right)_{0}+\left\|\overline{\mathcal{N}}_{t}^{1} \bar{v}_{t}\right\|_{0}^{2}+2\left(\bar{v}_{t}, \overline{\mathfrak{J}}_{t, z}^{1} \bar{v}_{t}\right)_{0}+\int_{D^{1} \cup E^{1}}\left\|\overline{\mathcal{I}}_{t, z}^{1} \bar{v}_{t}\right\|_{0}^{2} \pi^{1}(d z)\right) d t \\
& +\left(2\left(\tilde{v}_{t}, b_{t}^{i} \partial_{i} \tilde{v}_{t}+\bar{c}_{t}^{\bar{l}} \tilde{v}_{t}^{\bar{l}}\right)_{0}+2\left(\tilde{v}_{t}, \overline{\mathfrak{\Omega}}_{t}^{2} \tilde{v}_{t}\right)_{0}+2\left(\tilde{v}_{t}, \overline{\mathfrak{J}}_{t, z}^{2} \tilde{v}_{t}\right)_{0}\right) d t+2\left(v_{t}, \overline{\mathcal{N}}_{t}^{1 ; \varrho} \tilde{v}_{t}\right)_{0} d w_{t}^{1 ; \varrho} \\
& +\int_{Z^{1}}\left(2\left(\tilde{v}_{t-}, \overline{\mathcal{I}}_{t, z}^{1} \tilde{v}_{t-}\right)_{0}+\left\|\overline{\mathcal{I}}_{t, z}^{1} \tilde{v}_{t-}\right\|_{0}^{2}\right) q^{1}(d t, d z), \quad \tau_{n}<t \leq \tau_{n+1}, \\
\left\|\bar{v}_{t}\right\|_{0}^{2}= & 0, \quad t \leq \tau_{n}, \quad l \in\left\{1, \ldots, d_{2}\right\},
\end{aligned}
$$

where all coefficients and operators are defined as in (2.1) with the following changes: 
(1) for each $k \in\{1,2\}, v^{k}$ is replaced with

$$
\bar{v}^{k ; \bar{l}}:=v^{k ; \bar{l}}+\mathbf{1}_{\{2\}}(\alpha) \lambda \sigma^{k ; i \varrho} r_{1}^{-2} x^{i} \delta_{l \bar{l}}
$$

(2) for each $k \in\{1,2\}, \rho^{k}$ replaced with

$$
\bar{\rho}^{k: l \bar{l}}:=\rho^{k: l \bar{l}}+\left(\frac{r_{1}^{\lambda}\left(\tilde{H}^{k}\right)}{r_{1}^{\lambda}}-1\right)\left(I_{d_{2}}^{\bar{l}}+\rho^{k ; \bar{l}}\right) ;
$$

(3) $c$ is replaced with

$$
\begin{gathered}
\bar{c}^{\bar{l}}=c^{l \bar{l}}+\lambda b^{i} r^{-2} x^{i} \delta_{l \bar{l}}+\sum_{k=1}^{2} \lambda^{2} \sigma^{k ; \varrho} \sigma^{k ; j \varrho} r_{1}^{-4} x^{i} x^{j} \\
+\sum_{k=1}^{2} \int_{D^{k}}\left(\left(\frac{r_{1}^{\lambda}}{r_{1}^{\lambda}\left(\tilde{H}^{k ;-1}\right)}-1\right)\left(I_{m}^{l \bar{l}}+\rho^{k}\left(\tilde{H}^{k ;-1}\right)\right)-\mathbf{1}_{(1,2]}(\alpha) \lambda r_{1}^{-2} x_{i} H^{k ; i}\left(\tilde{H}^{k ;-1}\right)\right) \pi^{k}(d z) .
\end{gathered}
$$

Since for all $k, \omega$ and $t,\left|r_{1}^{-1} \sigma^{k}\right|_{0}+\left|r_{1}^{-1} \nabla \sigma^{k}\right|_{\bar{\beta}-1}+\left|v^{k}\right|_{\tilde{\beta}} \leq N_{0}$, for $\bar{\beta}>1 \vee \alpha$ and $\tilde{\beta}>\alpha$, it is clear that $\left|\bar{v}^{k}\right|_{\alpha} \leq N$. Moreover, since for all $k, \omega$ and $t,\left|r_{1}^{-1} H^{k}\right|_{0}+\left|H^{k}\right|_{\bar{\beta}} \leq K^{k}$ and $|\rho|_{\tilde{\beta}^{\prime}} \leq l^{k}$, applying the estimates in Lemma (4.10) (1), we get

$$
\left|\bar{\rho}^{k}\right|_{\alpha} \leq l^{k}+K^{k}\left(1+l^{k}\right) \text { and }|c|_{\alpha} \leq N_{0} .
$$

We will now estimate the drift terms of (3.5) in terms of $\left\|\bar{v}_{t}\right\|_{0}^{2}$. We write $f \sim g$ if $\int_{\mathbf{R}^{d_{1}}}|f(x)| d x$ $=\int_{\mathbf{R}^{\mathbf{d}_{1}}}|g(x)| d x$ and $f \ll g$ if $\int_{\mathbf{R}^{d_{1}}}|f(x)| d x \leq \int_{\mathbf{R}^{\mathbf{d}_{1}}}|g(x)| d x$. Using the divergence theorem, for any $v: \mathbf{R}^{d_{1}} \rightarrow \mathbf{R}^{d_{2}}, \sigma: \mathbf{R}^{d_{1}} \rightarrow \mathbf{R}^{d_{1}}$ and $v: \mathbf{R}^{d_{1}} \rightarrow \mathbf{R}^{2 d_{2}}$ and all $x$, we get

$$
\begin{aligned}
& \sigma^{i} \sigma^{j} v^{l} v_{i j}^{l} \sim \frac{1}{2}\left(\sigma^{i} \sigma^{j}\right)_{i j} v-\sigma^{i} \sigma^{j} v_{i}^{l} v_{j}^{l}=\left(\sigma_{i j}^{i} \sigma^{j}+\sigma_{j}^{i} \sigma_{i}^{j}\right)|v|^{2}-\sigma^{i} \sigma^{j} v_{i}^{l} v_{j}^{l}, \\
& 2 \sigma_{j}^{i} \sigma^{j} v^{l} v_{i}^{l} \sim-\left(\sigma_{j}^{i} \sigma^{j}\right)_{i}|v|^{2}=\left(\sigma_{i j}^{i} \sigma^{j}+\sigma_{j}^{i} \sigma_{i}^{j}\right)|v|^{2}
\end{aligned}
$$

and

$$
\sigma^{i} v^{l} v^{l \bar{l}} v_{i}^{\bar{l}}+\sigma^{i} v^{\bar{l}} v^{l \bar{l}} v_{i}^{l}=\sigma^{i} v^{l} v_{\text {sym }}^{l \bar{l}} v_{i}^{\bar{l}} \sim-\left.\left(\sigma^{i} v_{\text {sym }}^{\bar{l}}\right)_{i}\left|v^{2}=-\left(\sigma_{i}^{i} v_{\text {sym }}^{l \bar{l}}+\sigma^{i} v_{\text {sym }}^{l \bar{l}}\right)\right| v\right|^{2},
$$

where $v_{\text {sym }}^{l \bar{l}}=\left(v^{l \bar{l}}+v^{\overline{l l}}\right) / 2$. Consequently, for all $\omega, t$, and $x$, we have

$$
2 \bar{v}^{l} \overline{\mathbf{Q}}^{1 ; l} \bar{v}+\left|\overline{\mathcal{N}}^{1} \bar{v}\right|^{2} \sim \frac{1}{2}\left(\left|\operatorname{div} \sigma^{1}\right|^{2}-\partial_{i} \sigma^{1 ; j \varrho} \partial_{j} \sigma^{1 ; i \varrho}\right)|\bar{v}|^{2}-\bar{v}_{\text {sym }}^{1 ; i \bar{\varrho}} \bar{v}^{l} \bar{v}^{\bar{l}} \operatorname{div} \sigma^{1 ; \varrho}+\left|\bar{v}^{1} \bar{v}\right|^{2} \ll N|\bar{v}|^{2}
$$

and

$$
2 \bar{v}^{l} \overline{\mathfrak{L}}^{(2) ; l} \bar{v} \ll-(1+\epsilon)\left|\sigma^{2 ; i} \partial_{i} \bar{v}\right|^{2}+N|\bar{v}|^{2},
$$

for any $\epsilon>0$, where in the last estimate we have also used Young's inequality. By Lemma 4.10 (2) and basic properties of the determinant, there is a constant $N=N\left(d, N_{0}, \eta^{1}, \eta^{2}\right)$ such that for all $k, \omega, t, x$, and $z$,

$$
\operatorname{det} \tilde{H}^{k ;-1}-1=\operatorname{det}\left(I_{d}+F^{k}\right)-1 \leq\left|\nabla F^{k}\right| \leq N\left|\nabla H^{k}\right|
$$


and

$$
\operatorname{det} \tilde{H}^{k,-1}-1-\operatorname{div} F^{k} \leq\left|\nabla F^{k}\right|^{2} \leq N\left|\nabla H^{k}\right|^{2} .
$$

Thus, integrating by parts, for all $\omega, t$, and $x$, we get

$$
\begin{aligned}
& 2 \bar{v}^{l} \overline{\mathfrak{I}}^{1 ; l} \bar{v}+ \int_{D^{1} \cup E^{1}}\left|\overline{\mathcal{I}}^{1} \bar{v}\right|^{2} \pi^{1}(d z) \sim 2 \int_{D^{1}} \bar{\rho}_{s y m}^{1 ; i \bar{l}}\left(\tilde{H}^{1 ;-1}\right)\left(\operatorname{det} \nabla \tilde{H}^{1 ;-1}-1\right) \pi^{1}(d z) \bar{v}^{\bar{l}} \bar{v}^{l} \\
&+\int_{D^{1} \cup E^{1}}\left(\operatorname{det} \nabla \tilde{H}^{1 ;-1}-1+\mathbf{1}_{(1,2]}(\alpha) \mathbf{1}_{D^{1}} \operatorname{div} F^{1}\right) \pi^{1}(d z)|\bar{v}|^{2} \\
&+\int_{D^{1} \cup E^{1}}\left(\mathbf{1}_{E^{1}} 2 \bar{\rho}_{s y m}^{1 ; \bar{l}}\left(\tilde{H}^{1 ;-1}\right) \bar{v}^{\bar{l}} \bar{v}^{l}+\left|\bar{\rho}^{1}\left(\tilde{H}^{1 ;-1}\right) \bar{v}\right|^{2}\right) \operatorname{det} \nabla \tilde{H}^{1 ;-1} \pi^{1}(d z) \\
& \ll N\left(\int_{D^{1}}\left(K^{1}(z)^{2}+l^{1}(z) K^{1}(z)+l^{1}(z)^{2}\right) \pi^{1}(d z)+\int_{E^{1}}\left(K^{k}(z)+l^{k}(z)\right) \pi^{1}(d z)\right)|\bar{v}|^{2} .
\end{aligned}
$$

Analogously, for all $\omega, t$, and $x$, we obtain

$$
2 \bar{v}^{l} \overline{\mathfrak{J}}^{2 ; l} \bar{v} \leq-(1+\epsilon) \int_{D^{2} \cup E^{2}}\left|\bar{v}\left(\tilde{H}^{2}\right)-\bar{v}\right|^{2} \pi^{2}(d z)+N|\bar{v}|^{2} .
$$

Therefore, combining the above estimates, $\mathbf{P}$-a.s. for all $t$,

$$
Q_{t} \leq N \int_{0}^{t} Q_{s} d s+M_{t}
$$

where $\left(M_{t}\right)_{t \leq T}$ is a càdlàg square-integrable martingale. Taking the expectation of (3.6) and applying Gronwall's lemma, we get $\sup _{t \leq T} \mathbf{E} Q_{t}=0$, which implies that $\mathbf{P}$-a.s. for all $t$ and $x$, $\tilde{v}_{t}(x)=0$. This completes the proof.

\subsection{Small jump case}

Set $\left(w^{\varrho}\right)_{\varrho \geq 1}=\left(w^{1 ; \varrho}\right)_{\varrho \geq 1},(Z, \mathcal{Z}, \pi)=\left(\mathcal{Z}^{1}, \mathcal{Z}^{1}, \pi^{1}\right), p(d t, d z)=p^{1}(d t, d z)$, and $q(d t, d z)=$ $q^{1}(d t, d z)$. Let $\sigma_{t}(x)=\left(\sigma_{t}^{i \varrho}(x)\right)_{1 \leq i \leq d_{1}, \varrho \geq 1}$ be a $\ell_{2}\left(\mathbf{R}^{d_{1}}\right)$-valued $\mathcal{R}_{T} \otimes \mathcal{B}\left(\mathbf{R}^{d_{1}}\right)$-measurable function defined on $\Omega \times[0, T] \times \mathbf{R}^{d_{1}}$ and $H_{t}(x, z)=\left(H_{t}^{i}(x, z)\right)_{1 \leq i \leq d_{1}}$ be a $\mathcal{P}_{T} \otimes \mathcal{B}\left(\mathbf{R}^{d_{1}}\right) \otimes \mathcal{Z}$-measurable function defined on $\Omega \times[0, T] \times \mathbf{R}^{d_{1}} \times Z$.

We introduce the following assumption for $\beta>1 \vee \alpha$.

Assumption $3.1(\beta)$. (1) There is a constant $N_{0}>0$ such that for all $(\omega, t) \in \Omega \times[0, T]$,

$$
\left|r_{1}^{-1} b_{t}\right|_{0}+\left|r_{1}^{-1} \sigma_{t}\right|_{0}+\left|\nabla b_{t}\right|_{\beta-1}+\left|\nabla \sigma_{t}\right|_{\beta-1} \leq N_{0} .
$$

Moreover, for all $(\omega, t, z) \in \Omega \times[0, T] \times Z$,

$$
\left|r_{1}^{-1} H_{t}(z)\right|_{0} \leq K_{t}(z) \quad \text { and } \quad\left|\nabla H_{t}(z)\right|_{\beta-1} \leq \bar{K}_{t}(z),
$$

where $K: \Omega \times[0, T] \times Z \rightarrow \mathbf{R}_{+}$is a $\mathcal{P}_{T} \otimes \mathcal{Z}$-measurable function satisfying

$$
K_{t}(z)+\bar{K}_{t}(z)+\int_{Z}\left(K_{t}(z)^{\alpha}+\bar{K}_{t}(z)^{2}\right) \pi(d z) \leq N_{0}
$$

for all $(\omega, t, z) \in \Omega \times[0, T] \times Z$. 
(2) There is a constant $\eta \in(0,1)$ such that for all $(\omega, t, x, z) \in\left\{(\omega, t, x, z) \in \Omega \times[0, T] \times \mathbf{R}^{d_{1}} \times\right.$ $\left.Z:\left|\nabla H_{t}(\omega, x, z)\right|>\eta\right\}$,

$$
\left|\left(I_{d_{1}}+\nabla H_{t}(x, z)\right)^{-1}\right| \leq N_{0}
$$

Let Assumption 3.1( $\beta$ ) hold for some $\beta>1 \vee \alpha$. Let $\tau \leq T$ be a stopping time. Consider the system of SIDEs on $[0, T] \times \mathbf{R}^{d_{1}}$ given by

$$
\begin{aligned}
d v_{t}(x) & =\left(\mathbf{1}_{\{2\}}(\alpha) \frac{1}{2} \sigma_{t}^{i \varrho}(x) \sigma_{t}^{j \varrho}(x) \partial_{i j} v_{t}(x)+\mathfrak{b}_{t}^{i}(x) \partial_{i} v_{t}(x)\right) d t+\mathbf{1}_{\{2\}}(\alpha) \sigma_{t}^{i \varrho}(x) \partial_{i} v_{t}(x) d w_{t}^{\varrho} \\
& +1_{(1,2]}(\alpha) \int_{Z}\left(v_{t}\left(x+H_{t}(x, z)\right)-v_{t}(x)+F_{t}(x, z) \partial_{i} v_{t}(x)\right) \pi(d z) d t \\
& +\int_{Z}\left(v_{t-}\left(x+H_{t}(x, z)\right)-v_{t-}(x)\right)\left[1_{(1,2]}(\alpha) q(d t, d z)+1_{[0,1]}(\alpha) p(d t, d z)\right], \quad \tau<t \leq T \\
v_{t}(x) & =x, \quad t \leq \tau
\end{aligned}
$$

where

$$
\mathfrak{b}_{t}^{i}(x):=\mathbf{1}_{[1,2]}(\alpha) b_{t}^{i}(x)+\mathbf{1}_{\{2\}}(\alpha) \sigma_{t}^{j \varrho}(x) \partial_{j} \sigma_{t}^{i \varrho}(x)
$$

and

$$
F_{t}(x, z):=-H_{t}\left(\tilde{H}_{t}^{-1}(x, z), z\right) .
$$

We associate with (3.7), the stochastic flow $Y_{t}=Y_{t}(\tau, x),(t, x) \in[0, T] \times \mathbf{R}^{d_{1}}$, generated by the SDE

$$
\begin{aligned}
d Y_{t}= & -\mathbf{1}_{[1,2]}(\alpha) b_{t}\left(Y_{t}\right) d t-\mathbf{1}_{\{2\}}(\alpha) \sigma_{t}^{\varrho}\left(Y_{t}\right) d w_{t}^{\varrho} \\
& +\int_{Z} F_{t}\left(Y_{t-}, z\right)\left[1_{(1,2]}(z) q(d t, d z)+1_{[0,1]}(z) p(d t, d z)\right], \quad \tau<t \leq T, \\
Y_{t}= & x, \quad t \leq \tau .
\end{aligned}
$$

Owing to parts (1) and (2) of Lemma 4.10, for each $\omega, t$, and $z$, the inverse of the mapping $\tilde{F}_{t}(x, z):=x+F_{t}(x, z)=x-H_{t}\left(\tilde{H}_{t}^{-1}(x, z), z\right)$ is $\tilde{H}_{t}(x, z):=x+H_{t}(x, z)$ and there is a constant $N=N\left(d_{1}, N_{0}, \beta, \eta\right)$ such that for all $\omega, t, x, y$, and $z$,

$$
\left|r_{1}^{-1} F_{t}(z)\right|_{0} \leq N K_{t}(z), \quad\left|\nabla F_{t}(z)\right|_{\beta-1} \leq K_{t}(z), \quad\left|\left(I_{d_{1}}+\nabla F_{t}(x, z)\right)^{-1}\right| \leq N .
$$

Thus, by Theorem 2.1 in [LM14b], there is a modification of the solution of (3.8), which we still denote by $Y_{t}=Y_{t}(\tau, x)$, that is a $C_{l o c}^{\beta^{\prime}}$-diffeomorphism for any $\beta^{\prime} \in[1, \beta)$. Moreover, P-a.s. Y. $(\tau, \cdot), Y^{-1}(\tau, \cdot) \in D\left([0, T] ; C_{l o c}^{\beta^{\prime}}\left(\mathbf{R}^{d_{1}} ; \mathbf{R}^{d_{1}}\right)\right)$, and $Y_{t-}^{-1}(\tau, \cdot)$ coincides with the inverse of $Y_{t-}(\tau, \cdot)$ for all $t$. The following proposition shows that the inverse flow $Y_{t}^{-1}(\tau)$ solves (3.7).

Proposition 3.1. Let Assumption 3.1( $\beta$ ) hold for some $\beta>1 \vee \alpha$. For each stopping time $\tau \leq T$ and $\beta^{\prime} \in[1 \vee \alpha, \beta), v_{t}(x)=v_{t}(\tau, x)=Y_{t}^{-1}(\tau, x)$ solves (3.7) and for each $\epsilon>0$ and $p \geq 2$, there is a constant $N=N\left(d_{1}, p, N_{0}, T, \beta^{\prime}, \eta, \epsilon\right)$ such that

$$
\mathbf{E}\left[\sup _{t \leq T}\left|r_{1}^{-(1+\epsilon)} v_{t}(\tau)\right|_{0}^{p}\right]+\mathbf{E}\left[\sup _{t \leq T}\left|r_{1}^{-\epsilon} \nabla v_{t}(\tau)\right|_{\beta^{\prime}-1}^{p}\right] \leq N .
$$


Proof. The estimate (3.9) is given in Theorem 2.1 in [LM14b] (see also Remark 2.1), so we only need to show that $Y_{t}^{-1}(\tau, x)$ solves (3.7). Let $\left(\delta_{n}\right)_{n \geq 1}$ be a sequence such that $\delta_{n} \in(0, \eta)$ for all $n$ and $\delta_{n} \rightarrow 0$ as $n \rightarrow \infty$. It is clear that there is a constant $N=N\left(N_{0}\right)$ such that for all $\omega$ and $t$,

$$
\pi\left(\left\{z: K_{t}(z)>\delta_{n}\right\}\right) \leq \frac{N}{\delta_{n}^{\alpha}} .
$$

For each $n$, consider the system of SIDEs on $[0, T] \times \mathbf{R}^{d_{1}}$ given by

$$
\begin{gathered}
d v_{t}^{(n)}(x)=\left(\mathbf{1}_{\{2\}}(\alpha) \frac{1}{2} \sigma_{t}^{i \varrho}(x) \sigma_{t}^{j \varrho}(x) \partial_{i j} v_{t}^{(n)}(x)+\mathfrak{b}_{t}^{i}(x) \partial_{i} v_{t}^{(n)}(x)\right) d t \\
+1_{(1,2]}(\alpha) \int_{Z} \mathbf{1}_{\left\{K_{t}>\delta_{n}\right\}}(z)\left(v_{t}^{(n)}\left(x+H_{t}(x, z)\right)-v_{t}^{(n)}(x)+F_{t}^{i}(x, z) \partial_{i} v_{t}^{(n)}(x)\right) \pi(d z) d t \\
+\int_{Z} \mathbf{1}_{\left\{K_{t}>\delta_{n}\right\}}(z)\left(v_{t-}^{(n)}\left(x+H_{t}(x, z)\right)-v_{t-}^{(n)}(x)\right)\left[1_{(1,2]}(\alpha) q(d t, d z)+1_{[0,1]}(\alpha) p(d t, d z)\right] \\
+\mathbf{1}_{\{2\}}(\alpha) \sigma_{t}^{i \varrho}(x) \partial_{i} v_{t}^{(n)}(x) d w_{t}^{\varrho}, \quad \tau<t \leq T, \quad v_{t}^{(n)}(x)=x, \quad t \leq \tau
\end{gathered}
$$

and the stochastic flow $Y_{t}^{(n)}=Y_{t}^{(n)}(\tau, x),(t, x) \in[0, T] \times \mathbf{R}^{d_{1}}$, generated by the SDE

$$
\begin{aligned}
d Y_{t}^{(n)}= & -\mathbf{1}_{[1,2]}(\alpha) b_{t}\left(Y_{t}^{(n)}\right) d t-\mathbf{1}_{\{2\}}(\alpha) \sigma_{t}^{\varrho}\left(Y_{t}^{(n)}\right) d w_{t}^{\varrho} \\
& +\int_{Z} \mathbf{1}_{\left\{K_{t}>\delta_{n}\right\}}(z) F_{t}\left(Y_{t-}^{(n)}, z\right)\left[1_{(1,2]}(\alpha) q(d t, d z)+1_{[0,1]}(\alpha) p(d t, d z)\right], \quad \tau<t \leq T, \\
Y_{t}^{(n)}(x)= & x, \quad t \leq \tau .
\end{aligned}
$$

Since (3.10) holds, we can rewrite equation (3.12) as

$$
\begin{aligned}
d Y_{t}^{(n)}= & -\left(\mathbf{1}_{[1,2]}(\alpha) b_{t}\left(Y_{t}^{(n)}\right)+1_{(1,2]}(\alpha) \int_{Z} \mathbf{1}_{\left\{K_{t}>\delta_{n}\right\}}(z) F_{t}\left(Y_{t}^{(n)}, z\right) \pi(d z)\right) d t \\
& -\mathbf{1}_{\{2\}}(\alpha) \sigma_{t}^{\varrho}\left(Y_{n}(t)\right) d w_{t}^{\varrho}+\int_{Z} \mathbf{1}_{\left\{K_{t}>\delta_{n}\right\}}(z) F_{t}\left(Y_{t-}^{(n)}, z\right) p(d t, d z), \quad \tau<t \leq T,
\end{aligned}
$$

and (3.11) as

$$
\begin{aligned}
d v_{t}^{(n)}(x)= & \left(\mathbf{1}_{\{2\}}(\alpha) \frac{1}{2} \sigma_{t}^{i \varrho}(x) \sigma_{t}^{j \varrho}(x) \partial_{i j} v_{t}^{(n)}(x)+\mathfrak{b}_{t}^{i}(x) \partial_{j} \sigma_{t}^{i \varrho}(x)\right) d t \\
& +\mathbf{1}_{\{2\}}(\alpha) \sigma_{t}^{i \varrho}(x) \partial_{i} v_{t}^{(n)}(x) d w_{t}^{\varrho}+1_{(1,2\}}(\alpha) \int_{Z} \mathbf{1}_{\left\{K_{t}>\delta_{n}\right\}}(z) F_{t}^{i}(x, z) \pi(d z) \partial_{i} v_{t}^{(n)}(x) d t \\
& +\int_{Z} \mathbf{1}_{\left\{K_{t}>\delta_{n}\right\}}(z)\left(v_{t-}^{(n)}\left(x+H_{t}(x, z)\right)-v_{t-}^{(n)}(x)\right) p(d t, d z), \quad \tau<t \leq T
\end{aligned}
$$

We claim that the solution $Y_{t}^{(n)}=Y_{t}^{(n)}(x)$ of (3.13) can be written as the solution of continuous SDEs with a finite number of jumps interlaced. Indeed, for each $n$ and stopping time $\tau^{\prime} \leq T$, 
consider the stochastic flow $\tilde{Y}_{t}^{(n)}=\tilde{Y}_{t}^{(n)}\left(\tau^{\prime}, x\right),(t, x) \in[0, T] \times \mathbf{R}^{d_{1}}$, generated by the SDE

$$
\begin{aligned}
d \tilde{Y}_{t}^{(n)}= & -\left[\mathbf{1}_{[1,2]}(\alpha) b_{t}\left(\tilde{Y}_{t}^{(n)}\right)+1_{(1,2]}(\alpha) \int_{Z} \mathbf{1}_{\left\{K>\delta_{n}\right\}}(t, z) F_{t}\left(\tilde{Y}_{t}^{(n)}, z\right) \pi(d z)\right] d t \\
& -\mathbf{1}_{\{2\}}(\alpha) \sigma_{t}^{\varrho}\left(\tilde{Y}_{t}^{(n)}\right) d w_{t}^{\varrho}, \tau^{\prime}<t \leq T, \\
\tilde{Y}_{t}^{(n)}= & x, \quad t \leq \tau^{\prime} .
\end{aligned}
$$

By Theorems 2.1 and 2.4 and Remark 2.2 in [LM14b], there is a modification of $\tilde{Y}_{t}^{(n)}=\tilde{Y}_{t}^{(n)}$ $\left(\tau^{\prime}, x\right)$, still denoted $\tilde{Y}_{t}^{(n)}\left(\tau^{\prime}, x\right)$, that is a $C_{l o c}^{\beta^{\prime}}$-diffeomorphism. Furthermore, P-a.s. we have that

$$
\tilde{Y}^{(n)}\left(\tau^{\prime}, \cdot\right), \tilde{Y}^{(n) ;-1}\left(\tau^{\prime}, \cdot\right) \in C\left([0, T] ; C_{l o c}^{\beta^{\prime}}\right)
$$

and $\tilde{v}_{t}^{(n)}=\tilde{v}_{t}^{(n)}\left(\tau^{\prime}, x\right)=\tilde{Y}_{t}^{(n) ;-1}\left(\tau^{\prime}, x\right)$ solves the SPDE given by

$$
\begin{aligned}
d \tilde{v}_{t}^{(n)}(x)= & \left(\mathbf{1}_{\{2\}}(\alpha) \frac{1}{2} \sigma_{t}^{i \varrho}(x) \sigma_{t}^{j \varrho}(x) \partial_{i j} v_{t}^{(n)}(x)+\mathfrak{b}_{t}^{i}(x) \partial_{i} v_{t}^{(n)}(x)\right) d t \\
& +\mathbf{1}_{\{2\}}(\alpha) \sigma_{t}^{i \varrho}(x) \partial_{i} v_{t}^{(n)}(x) d w_{t}^{\varrho} \\
& +1_{(1,2]}(\alpha) \int_{Z} \mathbf{1}_{\left\{K>\delta_{n}\right\}}(t, z) F^{i}(t, z) \pi(d z) d t \partial_{i} v_{t}^{(n)}(x), \quad \tau^{\prime}<t \leq T, \\
\tilde{v}_{t}^{(n)}(x)= & x, \quad t \leq \tau^{\prime}
\end{aligned}
$$

For each $n$, let

$$
A_{t}^{(n)}=\int_{] 0, t]} \int_{Z} \mathbf{1}_{\left\{K_{s}>\delta_{n}\right\}}(z) p(d s, d z), \quad t \geq 0,
$$

and define the sequence of stopping times $\left(\tau_{l}^{(n)}\right)_{l=1}^{\infty}$ recursively by $\tau_{0}^{(n)}=\tau$ and

$$
\tau_{l+1}^{(n)}=\inf \left\{t>\tau_{l}^{(n)}: \Delta A_{t}^{(n)} \neq 0\right\} \wedge T
$$

Fix some $n \geq 1$. It is clear that $\mathbf{P}$-a.s. for all $x$ and $t \in\left[0, \tau_{1}^{(n)}\right)$,

$$
Y_{t}^{(n) ;-1}(\tau, x)=\tilde{Y}_{t}^{(n) ;-1}(\tau, x)=\tilde{v}_{t}^{(n)}(\tau, x)
$$

satisfies (3.14) up to, but not including time $\tau_{1}^{(n)}$. Moreover, P-a.s. for all $x$,

$$
Y_{\tau_{1}^{(n)}}^{(n)}(\tau, x)=\tilde{Y}_{\tau_{1}^{n^{n}}}^{(n)}(\tau, x)+\int_{Z} F_{\tau_{1}^{(n)}}\left(\tilde{Y}_{\tau_{1}^{(n)}-}^{(n)}(\tau, x), z\right) p\left(\left\{\tau_{1}^{(n)}\right\}, d z\right),
$$

and hence

$$
Y_{\tau_{1}^{(n)}}^{(n)-1}(\tau, x)=\int_{Z} \tilde{v}_{\tau_{1}^{(n)}-}^{(n)}\left(\tau, x+H_{\tau_{1}^{(n)}}(x, z)\right) p\left(\left\{\tau_{1}^{(n)}\right\}, d z\right)
$$

Consequently, $v_{t}^{(n)}(\tau, x)=Y_{t}^{(n) ;-1}(\tau, x)$ solves (3.14) up to and including time $\tau_{1}^{(n)}$. Assume that for some $l \geq 1, v_{t}^{(n)}(\tau, x)=Y_{t}^{(n) ;-1}(\tau, x)$ solves (3.14) up to and including time $\tau_{l}^{(n)}$. Clearly, 
P-a.s. for all $x$ and $t \in\left[\tau_{l}^{(n)}, \tau_{l+1}^{(n)}\right), Y_{t}^{(n)}(x)=\tilde{Y}_{t}^{(n)}\left(\tau_{l}^{(n)}, Y_{\tau_{l}^{(n)}-}^{(n)}(x)\right)$, and thus P-a.s. for all $x$ and $t \in\left[\tau_{l}^{(n)}, \tau_{l+1}^{(n)}\right)$

$$
Y_{t}^{(n) ;-1}(x)=\tilde{Y}_{t}^{(n)}\left(\tau_{l}^{(n)}, Y_{\tau_{l}^{(n)}-}^{(n)}(x)\right)=\tilde{v}_{t}^{(n)}\left(\tau_{l}^{(n)}, Y_{\tau_{l}^{(n)}-}^{(n)}(x)\right) .
$$

Moreover, P-a.s. for all $x$,

$$
Y_{n}^{-1}\left(\tau_{l+1}^{n}, x\right)=\int_{U} \tilde{v}_{n}\left(\tau_{l}^{n}, \tau_{l+1}^{n}-, x+H\left(\tau_{l+1}^{n}, x, z\right)\right) p\left(\left\{\tau_{l+1}^{n}\right\}, d z\right)
$$

which implies that $v_{t}^{(n)}(\tau, x)=Y_{t}^{(n) ;-1}(\tau, x)$ solves (3.14) up to and including time $\tau_{l+1}^{n}$. Therefore, by induction, for each $n, v_{t}^{(n)}(\tau, x)=Y_{t}^{(n) ;-1}(\tau, x)$ solves (3.14). It is easy to see that for all $\omega, t$, and $z$

$$
\left|r_{1}^{-1} \mathbf{1}_{\left\{K_{t}>\delta_{n}\right\}}(z) F_{t}(z)-r_{1}^{-1} F_{t}(z)\right|_{0}+\left|\mathbf{1}_{\left\{K_{t}>\delta_{n}\right\}}(z) \nabla F_{t}(z)-\nabla F_{t}(z)\right|_{\beta-1} \leq \mathbf{1}_{\left\{K_{t} \leq \delta_{n}\right\}}(z) K_{t}(z)
$$

and thus

$$
d \mathbf{P} d t-\lim _{n \rightarrow \infty} \int_{D} \mathbf{1}_{\left\{K \leq \delta_{n}\right\}}(t, z) K_{t}(z)^{2} \pi(d z)+d \mathbf{P} d t-\lim _{n \rightarrow \infty} \int_{E} \mathbf{1}_{\left\{K \leq \delta_{n}\right\}}(t, z) K_{t}(z) \pi(d z)=0 .
$$

By virtue of Theorem 2.3 in [LM14b], for each $\epsilon>0$, and $p \geq 2$, we have

$$
\begin{gathered}
\lim _{n \rightarrow \infty}\left(\mathbf{E}\left[\sup _{t \leq T} \mid r_{1}^{-(1+\epsilon)}\left(Y_{t}^{(n)}(\tau)-\left.r_{1}^{-(1+\epsilon)} Y_{t}(\tau)\right|_{0} ^{p}\right]+\mathbf{E}\left[\sup _{t \leq T}\left|r_{1}^{-\epsilon} \nabla Y_{t}^{(n)}(\tau)-r_{1}^{-\epsilon} \nabla Y_{t}(\tau)\right|_{\beta^{\prime}-1}^{p}\right]\right)=0,\right. \\
\lim _{n \rightarrow \infty} \mathbf{E}\left[\sup _{t \leq T}\left|r_{1}^{-(1+\epsilon)} Y_{t}^{(n) ;-1}(\tau)-r_{1}^{-(1+\epsilon)} Y_{t}^{-1}(\tau)\right|_{0}^{p}\right]=0
\end{gathered}
$$

and

$$
\lim _{n \rightarrow \infty} \mathbf{E}\left[\sup _{t \leq T}\left|r_{1}^{-\epsilon} \nabla Y_{t}^{(n) ;-1}(\tau)-r_{1}^{-\epsilon} \nabla Y_{t}^{-1}(\tau)\right|_{\beta^{\prime}-1}^{p}\right]=0
$$

Then passing to the limit in both sides of (3.11) and making use of Assumption 3.1( $\beta$ ), the estimate (3.4), and basic convergence properties of stochastic integrals, we find that $v_{t}(\tau, x)=X_{t}^{-1}(\tau, x)$ solves $(3.7)$.

\subsection{Adding free and zero-order terms}

Set $\left(w^{\varrho}\right)_{\varrho \geq 1}=\left(w^{1 ; \varrho}\right)_{\varrho \geq 1},(Z, \mathcal{Z}, \pi)=\left(\mathcal{Z}^{1}, \mathcal{Z}^{1}, \pi^{1}\right), p(d t, d z)=p^{1}(d t, d z)$, and $q(d t, d z)=$ $p^{1}(d t, d z)-\pi^{1}(d z) d t$. Also, set $D=D^{1}, E=E^{1}$, and assume $Z=D \cup E$. Let $v_{t}(x)=$ $\left(v_{t}^{l \bar{l} \varrho}(\omega, x)\right)_{1 \leq l, \bar{l} \leq d_{2}, \varrho \geq 1}$ be a $\ell_{2}\left(\mathbf{R}^{2 d_{2}}\right)$-valued $\mathcal{R}_{T} \otimes \mathcal{B}\left(\mathbf{R}^{d_{1}}\right)$-measurable function defined on $\Omega \times$ $[0, T] \times \mathbf{R}^{d_{1}}$ and $\rho_{t}(x, z)=\left(\rho_{t}^{l \bar{l}}(\omega, x, z)\right)_{1 \leq l, \bar{l} \leq d_{2}}$ be a $\mathcal{P}_{T} \otimes \mathcal{B}\left(\mathbf{R}^{d_{1}}\right) \otimes \mathcal{Z}$-measurable function defined on $\Omega \times[0, T] \times \mathbf{R}^{d_{1}} \times Z$.

We introduce the following assumptions for $\beta>1 \vee \alpha$ and $\tilde{\beta}>\alpha$. 
Assumption $3.2(\beta)$. (1) There is a constant $N_{0}>0$ such that for all $(\omega, t) \in \Omega \times[0, T]$,

$$
\left|r_{1}^{-1} b_{t}\right|_{0}+\left|r_{1}^{-1} \sigma_{t}\right|_{0}+\left|\nabla b_{t}\right|_{\beta-1}+\left|\nabla \sigma_{t}\right|_{\beta-1} \leq N_{0} .
$$

Moreover, for all $(\omega, t, z) \in \Omega \times[0, T] \times Z$,

$$
\left|r_{1}^{-1} H_{t}(z)\right|_{0} \leq K_{t}(z) \quad \text { and } \quad\left|\nabla H_{t}(z)\right|_{\beta-1} \leq \bar{K}_{t}(z) \text {, }
$$

where $K: \Omega \times[0, T] \times Z \rightarrow \mathbf{R}_{+}$is a $\mathcal{P}_{T} \otimes \mathcal{Z}$-measurable function satisfying

$$
K_{t}(z)+\bar{K}_{t}(z)+\int_{D}\left(K_{t}(z)^{\alpha}+\bar{K}_{t}(z)^{2}\right) \pi(d z)+\int_{E}\left(K_{t}(z)^{\alpha \wedge 1}+\bar{K}_{t}(z)\right) \pi(d z) \leq N_{0},
$$

for all $(\omega, t, z) \in \Omega \times[0, T] \times Z$.

(2) There is a constant $\eta \in(0,1)$ such that for all $(\omega, t, x, z) \in\left\{(\omega, t, x, z) \in \Omega \times[0, T] \times \mathbf{R}^{d_{1}} \times\right.$ $\left.Z:\left|\nabla H_{t}(\omega, x, z)\right|>\eta\right\}$

$$
\left|\left(I_{d_{1}}+\nabla H_{t}(x, z)\right)^{-1}\right| \leq N_{0} .
$$

Assumption $3.3(\tilde{\beta})$. There is a constant $N_{0}>0$ such that for all $(\omega, t) \in \Omega \times[0, T]$,

$$
\left|c_{t}\right|_{\tilde{\beta}}+\left|v_{t}\right|_{\tilde{\beta}}+\left|r_{1}^{-\theta} f_{t}\right|_{\tilde{\beta}}+\left|r_{1}^{-\theta} g_{t}\right|_{\tilde{\beta}} \leq N_{0}
$$

Moreover, for all $(\omega, t, z) \in \Omega \times[0, T] \times Z$,

$$
\left|\rho_{t}(z)\right|_{\tilde{\beta}}+\left|r_{1}^{-\theta} h_{t}(z)\right|_{\tilde{\beta}} \leq l_{t}(z),
$$

where $l: \Omega \times[0, T] \times Z \rightarrow \mathbf{R}_{+}$is a $\mathcal{P}_{T} \otimes \mathcal{Z}$-measurable function satisfying

$$
l_{t}(z)+\int_{D} l_{t}(z)^{2} \pi(d z)+\int_{E} l_{t}(z) \pi(d z) \leq N_{0} .
$$

$(\omega, t, z) \in \Omega \times[0, T] \times Z$.

Let Assumptions 3.2( $(\bar{\beta})$ and 3.3( $\tilde{\beta})$ hold for some $\bar{\beta}>1 \vee \alpha$ and $\tilde{\beta}>\alpha$. Let $\tau \leq T$ be a stopping time and $\varphi: \Omega \times \mathbf{R}^{d_{1}} \rightarrow \mathbf{R}^{d_{2}}$ be a $\mathcal{F}_{\tau} \otimes \mathcal{B}\left(\mathbf{R}^{d_{1}}\right)$-measurable random field. Consider the system of SIDEs on $[0, T] \times \mathbf{R}^{d_{1}}$ given by

$$
\begin{aligned}
d v_{t}^{l}= & \left(\mathcal{L}_{t}^{l} v_{t}+\hat{\mathrm{b}}_{t}^{i} \partial_{i} \phi^{l}+\hat{\mathrm{c}}_{t}^{l \bar{l}} \phi^{\bar{l}}+\hat{\mathfrak{f}}_{t}^{l}\right) d t+\left(\mathcal{N}_{t}^{l} \varrho\right. \\
& +\int_{Z}\left(\mathcal{I}_{t, z}^{l} v_{t-}+h_{t}^{l}(z)\right)\left[\mathbf{1}_{D}(z) q(d t, d z)+\mathbf{1}_{E}(z) p(d t, d z)\right], \quad \tau<t \leq T, \\
v_{t}^{l}= & \varphi^{l}, \quad t \leq \tau, \quad l \in\left\{1, \ldots, d_{2}\right\},
\end{aligned}
$$

where for $\phi \in C_{c}^{\infty}\left(\mathbf{R}^{d_{1}} ; \mathbf{R}^{d_{2}}\right)$ and $l \in\left\{1, \ldots, d_{2}\right\}$,

$$
\begin{aligned}
\mathcal{L}_{t}^{l} \phi(x):= & \mathbf{1}_{\{2\}}(\alpha) \frac{1}{2} \sigma_{t}^{i \varrho}(x) \sigma_{t}^{j \varrho}(x) \partial_{i j} \phi^{l}(x)+\mathbf{1}_{\{2\}}(\alpha) \sigma_{t}^{i \varrho}(x) a_{t}^{l \bar{l} \varrho}(x) \partial_{i} \phi^{\bar{l}}(x) \\
& +\int_{D^{k}} \rho_{t}^{l \bar{l}}(x, z)\left(\phi^{\bar{l}}\left(x+H_{t}(x, z)\right)-\phi^{\bar{l}}(x)\right) \pi(d z) \\
& +\int_{D^{k}}\left(\phi^{l}\left(x+H_{t}(x, z)\right)-\phi^{l}(x)-\mathbf{1}_{(1,2]}(\alpha) \partial_{i} \phi^{l}(x) H_{t}^{i}(x, z)\right) \pi(d z) \\
\mathcal{N}_{t}^{l \varrho} \phi^{l}(x):= & \mathbf{1}_{\{2\}}(\alpha) \sigma_{t}^{i \varrho}(x) \partial_{i} \phi^{l}(x)+v_{t}^{l \bar{l} \varrho}(x) \phi^{\bar{l}}(x), \\
\mathcal{I}_{t, z}^{l} \phi^{l}(x):= & \left(I_{d_{2}}+\rho_{t}^{l \bar{l}}(x, z)\right) \phi^{\bar{l}}\left(x+H_{t}(x, z)\right)-\phi^{l}(x),
\end{aligned}
$$


and where

$$
\begin{aligned}
\hat{\mathfrak{b}}_{t}^{i}(x):= & \mathbf{1}_{[1,2]}(\alpha) b_{t}^{i}(x)+\mathbf{1}_{\{2\}}(\alpha) \sigma_{t}^{j \varrho}(x) \partial_{j} \sigma_{t}^{i \varrho}(x) \\
& +\int_{D}\left(\mathbf{1}_{(1,2]}(\alpha) H_{t}^{i}(x, z)-H_{t}^{i}\left(\tilde{H}_{t}^{-1}(x, z), z\right)\right) \pi(d z), \\
\hat{\mathfrak{c}}_{t}^{l \bar{l}}(x):= & c_{t}^{l \bar{l}}(x)+\mathbf{1}_{\{2\}}(\alpha) \sigma_{t}^{j \varrho}(x) \partial_{j} v_{t}^{l \bar{l} \varrho}(x)+\int_{D}\left(\rho_{t}^{l \bar{l}}(x, z)-\rho_{t}^{l \bar{l}}\left(\tilde{H}_{t}^{-1}(x, z), z\right)\right) \pi(d z), \\
\hat{\mathfrak{f}}_{t}^{l}(x):= & f_{t}^{l}(x)+\mathbf{1}_{\{2\}}(\alpha) \sigma_{t}^{j \varrho}(x) \partial_{j} g_{t}^{l}(x)+\int_{D}\left(h_{t}^{l}(x, z)-h_{t}^{l}\left(\tilde{H}_{t}^{-1}(x, z), z\right)\right) \pi(d z) .
\end{aligned}
$$

We associate with (3.15) the stochastic flow $X_{t}=X_{t}(x)=X_{t}(\tau, x),(t, x) \in[0, T] \times \mathbf{R}^{d_{1}}$, given by (3.8). Let $\Gamma_{t}(x)=\Gamma_{t}(\tau, x),(t, x) \in[0, T] \times \mathbf{R}^{d_{1}}$, be the solution of the linear SDE given by

$$
\begin{aligned}
d \Gamma_{t}(x)= & \left(c_{t}\left(X_{t}(x)\right) \Gamma_{t}(x)+f_{t}\left(X_{t}(x)\right)\right) d t+\left(v_{t}^{\varrho}\left(X_{t}(x)\right) \Gamma_{t}(x)+g_{t}^{\varrho}\left(X_{t}(x)\right)\right) d w_{t}^{\varrho} \\
& +\int_{Z} \rho_{t}\left(\tilde{H}_{t}^{-1}\left(X_{t-}(x), z\right), z\right) \Gamma_{t-}(x)\left[\mathbf{1}_{D}(z) q(d t, d z)+\mathbf{1}_{E}(z) p(d t, d z)\right] \\
& +\int_{Z} h_{t}\left(\tilde{H}_{t}^{-1}\left(X_{t-}(x), z\right), z\right)\left[\mathbf{1}_{D}(z) q(d t, d z)+\mathbf{1}_{E}(z) p(d t, d z)\right], \quad \tau<t \leq T, \\
\Gamma_{t}(x)= & 0, \quad t \leq \tau .
\end{aligned}
$$

Let $\Psi_{t}(x)=\Psi_{t}(\tau, x),(t, x) \in[0, T] \times \mathbf{R}^{d_{1}}$, be the unique solution of the linear SDE given by

$$
\begin{aligned}
d \Psi_{t}(x)= & c_{t}\left(X_{t}(x)\right) \Psi_{t}(x) d t+v_{t}^{\varrho}\left(X_{t}(x)\right) \Phi_{t}(x) d w_{t}^{\varrho} \\
& +\int_{Z} \rho_{t}\left(\tilde{H}_{t}^{-1}\left(X_{t-}(x), z\right), z\right) \Psi_{t-}(x)\left[\mathbf{1}_{D}(z) q(d t, d z)+\mathbf{1}_{E}(z) p(d t, d z)\right], \quad \tau<t \leq T, \\
\Psi_{t}(x)= & I_{d_{2}}, \quad t \leq \tau .
\end{aligned}
$$

In the following lemma, we obtain $p$-th moment estimates of the weighted Hölder norms of $\Gamma$ and $\Psi$.

Lemma 3.2. Let Assumptions 3.2 $(\bar{\beta})$ and 3.3( $\tilde{\beta})$ hold for some $\bar{\beta}>1 \vee \alpha$ and $\tilde{\beta}>\alpha$. For each stopping time $\tau \leq T$ and $\beta^{\prime} \in[0, \bar{\beta} \wedge \tilde{\beta})$, there exists a $D\left([0, T], C_{l o c}^{\beta^{\prime}}\left(\mathbf{R}^{d_{1}} ; \mathbf{R}^{d_{2}}\right)\right)$-modification of $\Gamma(\tau)$ and $\Psi(\tau)$, also denoted by $\bar{\Gamma}(\tau)$ and $\Psi(\tau)$, respectively. Moreover, for each $\epsilon>0$ and $p \geq 2$, there is a constant $N=N\left(d_{1}, d_{2}, p, N_{0}, T, \beta^{\prime}, \eta, \epsilon, \theta\right)$ such that

$$
\mathbf{E}\left[\sup _{t \leq T}\left[\left|r_{1}^{-(\theta+\epsilon)} \Gamma_{t}(\tau)\right|_{\beta^{\prime}}^{p}\right]+\mathbf{E}\left[\sup _{t \leq T}\left|r_{1}^{-\epsilon} \Psi_{t}(\tau)\right|_{\beta^{\prime}}^{p}\right] \leq N\right.
$$

Proof. Let $\tau \leq T$ be a fixed stopping time and $\beta:=\bar{\beta} \wedge \tilde{\beta}$. Estimating (3.16) directly and using the Burkholder-Davis-Gundy inequality, Lemma 4.1, the multiplicative decomposition

$$
h_{t}\left(x, \tilde{H}_{t}^{-1}\left(X_{t-}(x), z\right), z\right)=r_{1}^{\theta}\left(X_{t-}(x)\right) \frac{r_{1}^{\theta}\left(\tilde{H}_{t}^{-1}\left(X_{t-}(x), z\right)\right)}{r_{1}^{\theta}\left(X_{t}(x)\right)} \frac{h_{t}\left(\tilde{H}_{t}^{-1}\left(X_{t-}(x), z\right), z\right)}{r_{1}^{\theta}\left(\tilde{H}_{t}^{-1}\left(X_{t-}(x), z\right)\right)},
$$


Hölder's inequality, Lemma 4.10 (1), Lemma 3.2 in [LM14b], and Gronwall's inequality, we get that for all $x$ and $y$,

$$
\mathbf{E}\left[\sup _{t \leq T}\left|\Gamma_{t}(x)\right|^{p}\right] \leq N r_{1}^{-\theta p}(x)
$$

and

$$
\mathbf{E}\left[\sup _{t \leq T}\left|\Gamma_{t}(x)-\Gamma_{t}(y)\right|^{p}\right] \leq N\left(r_{1}^{-p \theta}(x) \vee r_{1}^{-p \theta}(y)\right)|x-y|^{\left(\beta^{\prime} \wedge 1\right) p},
$$

where $N=N\left(d_{1}, p, N_{0}, T, \eta, \theta\right)$ is a positive constant. Now, assume that $[\beta]^{-} \geq 1$. As in the proof of Theorem 3.4 in [Kun04], it follows that $\mathfrak{U}_{t}=\nabla \Gamma_{t}(\tau, x)$ solves

$$
\begin{aligned}
d \mathfrak{U}_{t}= & \left(v_{t}^{\varrho}\left(X_{t}\right) \mathfrak{U}_{t}+\nabla v_{t}^{\varrho}\left(X_{t}\right) \nabla X_{t} \Gamma_{t}+\nabla g_{t}^{\varrho}\left(X_{t}\right) \nabla X_{t}\right) d w_{t}^{\varrho} \\
& +\int_{Z} \rho_{t}\left(\tilde{H}_{t}^{-1}\left(X_{t-}, z\right), z\right) \mathfrak{U}_{t-}\left[\mathbf{1}_{D}(z) q(d t, d z)+\mathbf{1}_{E}(z) p(d t, d z)\right] \\
& +\int_{Z} \nabla \rho_{t}\left(\tilde{H}_{t}^{-1}\left(X_{t-}, z\right), z\right) \nabla\left[\tilde{H}_{t}^{-1}\left(X_{t-}\right)\right] \Gamma_{t-}\left[\mathbf{1}_{D}(z) q(d t, d z)+\mathbf{1}_{E}(z) p(d t, d z)\right] \\
& \left.+\int_{Z} \nabla h_{t}\left(x, \tilde{H}_{t}^{-1}\left(X_{t}, z\right), z\right) \nabla\left[\tilde{H}_{t}^{-1}\left(X_{t-}\right)\right]\right]\left[\mathbf{1}_{D}(z) q(d t, d z)+\mathbf{1}_{E}(z) p(d t, d z)\right] \\
& +\left(c_{t}\left(X_{t}\right) \mathfrak{U}_{t}+\nabla c_{t}\left(X_{t}\right) \nabla X_{t} \Gamma_{t}+\nabla f_{t}\left(X_{t}\right) \nabla X_{t}\right) d t, \quad \tau<t \leq T, \\
\mathfrak{U}_{t}= & 0, t \leq \tau .
\end{aligned}
$$

Recall that by Lemma 4.6, a function $\phi: \mathbf{R}^{d_{1}} \rightarrow \mathbf{R}^{n}, n \geq 1$ satisfies $\left|r^{-\theta} \phi\right|_{\beta}<\infty$ if an only if $\left|r^{-\theta} \phi\right|_{0}, \ldots,\left|r^{-\theta} \partial^{\gamma} \phi\right|_{0},|\gamma| \leq[\beta]^{-}$, and $\left.\left[r^{-\theta} \partial^{\gamma} \phi\right]\right|_{\{\beta\}^{+}}$are finite. Estimating as above and using Proposition 3.4 in [LM14b], we obtain that for each $p \geq 2$, there is a constant $N=N\left(d_{1}, d_{2}, p, N_{0}, T, \theta\right)$ such that for all $x$ and $y$,

$$
\mathbf{E}\left[\sup _{t \leq T}\left|\nabla \Gamma_{t}(x)\right|^{p}\right] \leq r_{1}^{-p \theta}(x) N
$$

and

$$
\mathbf{E}\left[\sup _{t \leq T}\left|\nabla \Gamma_{t}(x)-\nabla \Gamma_{t}(y)\right|^{p}\right] \leq N\left(r_{1}^{-p \theta}(x) \vee r_{1}^{-p \theta}(y)\right)|x-y|^{((\beta-1) \wedge 1) p} .
$$

Using induction, we get that for each $p \geq 2$ and all multi-indices $\gamma$ with $0 \leq|\gamma| \leq[\beta]^{-}$and all $x$,

$$
\mathbf{E} \sup _{t \leq T}\left[\left|\partial^{\gamma} \Gamma_{t}(x)\right|^{p}\right] \leq r_{1}^{-p \theta}(x) N,
$$

and for all multi-indices $\gamma$ with $|\gamma|=[\beta]^{-}$and all $x, y$,

$$
\mathbf{E}\left[\sup _{t \leq T}\left|\partial^{\gamma} \Gamma_{t}(x)-\partial^{\gamma} \Gamma_{t}(y)\right|^{p}\right] \leq N\left(r_{1}^{-p \theta}(x) \vee r_{1}^{-p \theta}(y)\right)|x-y|^{\left(\beta-[\beta]^{-}\right) p},
$$

for a constant $N=N\left(d_{1}, d_{2}, p, N_{0}, T, \beta, \eta, \theta\right)$. It is also clear that for each $p \geq 2$ and all multi-indices $\gamma$ with $0 \leq|\gamma| \leq[\beta]^{-}$and all $x$,

$$
\mathbf{E}\left[\sup _{t \leq T}\left|\partial^{\gamma} \Psi_{t}(x)\right|^{p}\right] \leq N
$$


and for all multi-indices $\gamma$ with $|\gamma|=[\beta]^{-}$and all $x, y$,

$$
\mathbf{E}\left[\sup _{t \leq T}\left|\partial^{\gamma} \Psi_{t}(x)-\partial^{\gamma} \Psi_{t}(y)\right|^{p}\right] \leq N|x-y|^{\left(\beta-[\beta]^{-}\right) p} .
$$

We obtain the existence of a $D\left([0, T], C_{l o c}^{\beta^{\prime}}\left(\mathbf{R}^{d_{1}} ; \mathbf{R}^{d_{2}}\right)\right)$-modification of $\Gamma(\tau)$ and $\Psi(\tau)$ using estimate (3.17) and Corollary 5.4 in [LM14b]. This completes the proof.

Let $\tilde{\Phi}_{t}(x)=\tilde{\Phi}_{t}(\tau, x),(t, x) \in[0, T] \times \mathbf{R}^{d_{1}}$, be the solution of the linear SDE given by

$$
\begin{aligned}
d \tilde{\Phi}_{t}(x)= & \left(c_{t}\left(X_{t}(x)\right) \tilde{\Phi}_{t}(x)+f_{t}\left(X_{t}(x)\right)\right) d t+\left(v_{t}^{\varrho}\left(X_{t}(x)\right) \tilde{\Phi}_{t}(x)+g_{t}^{\varrho}\left(X_{t}(x)\right)\right) d w_{t}^{\varrho} \\
& +\int_{Z} \rho_{t}\left(\tilde{H}_{t}^{-1}\left(X_{t-}(x), z\right), z\right) \tilde{\Phi}_{t-}(x, y)\left[\mathbf{1}_{D}(z) q(d t, d z)+\mathbf{1}_{E}(z) p(d t, d z)\right] \\
& +\int_{Z} h_{t}\left(\tilde{H}_{t}^{-1}\left(X_{t-}(x), z\right), z\right)\left[\mathbf{1}_{D}(z) q(d t, d z)+\mathbf{1}_{E}(z) p(d t, d z)\right], \quad \tau<t \leq T, \\
\tilde{\Phi}_{t}(x)= & \varphi(x), \quad t \leq \tau .
\end{aligned}
$$

The following is a simple corollary of Lemma 3.2.

Corollary 3.3. Let Assumptions 3.2( $\bar{\beta})$ and 3.3( $\tilde{\beta})$ hold for some $\bar{\beta}>1 \vee \alpha$ and $\tilde{\beta}>\alpha$. For each stopping time $\tau \leq T$ and $\mathcal{F}_{\tau} \otimes \mathcal{B}\left(\mathbf{R}^{d_{1}}\right)$-measurable random field $\varphi$ such that for some $\beta^{\prime} \in[0, \bar{\beta} \wedge \tilde{\beta})$, P-a.s. $\varphi \in C_{l o c}^{\beta^{\prime}}\left(\mathbf{R}^{d_{1}} ; \mathbf{R}^{d_{2}}\right)$, there is a $D\left([0, T] ; C_{l o c}^{\beta^{\prime}}\left(\mathbf{R}^{d_{1}}, \mathbf{R}^{d_{2}}\right)\right)$-modification of $\tilde{\Phi}(\tau)$, also denoted by $\tilde{\Phi}(\tau)$, and $\mathbf{P}$-a.s. for all $(t, x) \in[0, T] \times \mathbf{R}^{d_{1}}$,

$$
\tilde{\Phi}_{t}(\tau, x)=\Psi_{t}(x) \varphi(x)+\Gamma_{t}(x) .
$$

Moreover, if for some $\theta^{\prime} \geq 0$ and $\beta^{\prime} \in[0, \bar{\beta} \wedge \tilde{\beta})$, $\mathbf{P}$-a.s. $r_{1}^{-\theta^{\prime}} \varphi \in C^{\beta^{\prime}}\left(\mathbf{R}^{d_{1}} ; \mathbf{R}^{d_{2}}\right)$, then for each $\epsilon>0$ and $p \geq 2$, there is a constant $N=N\left(d_{1}, d_{2}, p, N_{0}, T, \theta, \theta^{\prime}, \beta^{\prime}, \epsilon\right)$ such that

$$
\mathbf{E}\left[\sup _{t \leq T}\left|r_{1}^{-\left(\theta \vee \theta^{\prime}\right)-\epsilon} \tilde{\Phi}_{t}(\tau)\right|_{\beta^{\prime}}^{p} \mid \mathcal{F}_{\tau}\right] \leq N\left(\left|r_{1}^{-\theta^{\prime}} \varphi\right|_{\beta^{\prime}}^{p}+1\right) .
$$

Now we are ready to state our main result concerning fully-degenerate SIDEs and their connection with linear transformations of inverse flows of jump SDEs.

Proposition 3.4. Let Assumptions 3.2( $\bar{\beta})$ and 3.3( $\tilde{\beta})$ hold for some $\bar{\beta}>1 \vee \alpha$ and $\tilde{\beta}>\alpha$. For each stopping time $\tau \leq T$ and $\mathcal{F}_{\tau} \otimes \mathcal{B}\left(\mathbf{R}^{d_{1}}\right)$-measurable random field $\varphi$ such that for some $\beta^{\prime} \in(\alpha, \bar{\beta} \wedge \tilde{\beta})$ and $\theta^{\prime} \geq 0$, P-a.s. $r_{1}^{-\theta^{\prime}} \varphi \in C^{\beta^{\prime}}\left(\mathbf{R}^{d_{1}} ; \mathbf{R}^{d_{2}}\right)$, we have that $\mathbf{P}$-a.s. $\tilde{\Phi}\left(\tau, X^{-1}(\tau)\right) \in$ $D\left([0, T] ; C_{\text {loc }}^{\beta^{\prime}}\left(\mathbf{R}^{d_{1}} ; \mathbf{R}^{d_{2}}\right)\right)$ and $v_{t}(x)=v_{t}(\tau, x)=\tilde{\Phi}_{t}\left(\tau, X_{t}^{-1}(\tau, x)\right)$ solves $(3.15)$. Moreover, for each $\epsilon>0$ and $p \geq 2$,

$$
\mathbf{E}\left[\sup _{t \leq T}\left|r_{1}^{-\left(\theta \vee \theta^{\prime}\right)-\epsilon} v_{t}(\tau)\right|_{\beta^{\prime}}^{p} \mid \mathcal{F}_{\tau}\right] \leq N\left(\left|r_{1}^{-\theta^{\prime}} \varphi\right|_{\beta^{\prime}}^{p}+1\right),
$$

for a constant $N=N\left(d_{1}, d_{2}, p, N_{0}, T, \beta^{\prime}, \eta, \epsilon, \theta, \theta^{\prime}\right)$. 
Proof. Fix a stopping time $\tau \leq T$ and random field $\varphi$ such that for some $\beta^{\prime} \in(\alpha, \bar{\beta} \wedge \tilde{\beta})$ and $\theta^{\prime} \geq 0$, P-a.s. $r_{1}^{-\theta^{\prime}} \varphi \in C^{\beta^{\prime}}\left(\mathbf{R}^{d_{1}} ; \mathbf{R}^{d_{2}}\right)$. By virtue of Corollary 3.3 and Theorem 2.1 in [LM14b], P-a.s.

$$
\tilde{\Phi}\left(\tau, X^{-1}(\tau)\right) \in D\left([0, T] ; C_{l o c}^{\beta^{\prime}}\left(\mathbf{R}^{d_{1}}, \mathbf{R}^{d_{2}}\right)\right) .
$$

Then using the Ito-Wenzell formula (Proposition 4.16) and following a simple calculation, we obtain that $v_{t}(\tau, x):=\tilde{\Phi}_{t}\left(\tau, X_{t}^{-1}(\tau, x)\right)$ solves (3.15). By Theorem 2.1 in [LM14b] and Corollary 3.3, for each $\epsilon>0$ and $p \geq 2$, there exists a constant $N=N\left(d_{1}, p, N_{0}, T, \beta^{\prime}, \eta, \epsilon\right)$ such that

$$
\mathbf{E}\left[\sup _{t \leq T}\left|r_{1}^{-(1+\epsilon)} X_{t}^{-1}(\tau)\right|_{\beta^{\prime}}^{p}\right]+\mathbf{E}\left[\sup _{t \leq T}\left|r_{1}^{-\epsilon} \nabla X_{t}^{-1}(\tau)\right|_{\beta^{\prime}-1}^{p}\right] \leq N .
$$

Therefore applying Lemma 4.9 and Hölder's inequalty and using the estimates (3.20) and (3.18), we obtain (3.19), which completes the proof.

\subsection{Adding uncorrelated part (Proof of Theorem 2.2)}

Proof of Theorem 2.2. Fix a stopping time $\tau \leq T$ and random field $\varphi$ such that for some $\beta^{\prime} \in(\alpha, \bar{\beta} \wedge \tilde{\beta})$ and $\theta^{\prime} \geq 0$, P-a.s. $r_{1}^{-\theta^{\prime}} \varphi \in C^{\beta^{\prime}}\left(\mathbf{R}^{d_{1}} ; \mathbf{R}^{d_{2}}\right)$. Consider the system of SIDEs given by

$$
\begin{aligned}
d \tilde{v}_{t}^{l}= & \left(\left(\mathcal{L}_{t}^{1 ; l}+\mathcal{L}_{t}^{2 ; l}\right) \tilde{v}_{t}+\mathbf{1}_{[1,2]}(\alpha) \hat{b}_{t}^{i} \partial_{i} u_{t}^{l}+\hat{c}_{t}^{l \bar{l}} u_{t}^{\bar{l}}(x)+\hat{f}_{t}^{l}\right) d t+\left(\mathcal{N}_{t}^{1 ; l o} \tilde{v}_{t}+g_{t}^{l \varrho}\right) d w_{t}^{1 ; \varrho} \\
& +\mathcal{N}_{t}^{2 ; l \varrho} \tilde{v}_{t} d w_{t}^{2 ; \varrho}+\int_{Z^{1}}\left(\mathcal{I}_{t, z}^{1 ; l} \tilde{v}_{t-}+h_{t}^{l}(z)\right)\left[\mathbf{1}_{D^{1}}(z) q^{1}(d t, d z)+\mathbf{1}_{E^{1}} p^{1}(d t, d z)\right] \\
& +\int_{Z^{2}} \mathcal{I}_{t, z}^{2 ; l} \tilde{v}_{t-}\left[\mathbf{1}_{D^{2}}(z) q^{2}(d t, d z)+\mathbf{1}_{E^{2}}(z) p^{2}(d t, d z)\right] \quad \tau<t \leq T, \\
\tilde{v}_{t}^{l}= & \varphi^{l}, \quad t \leq \tau, \quad l \in\left\{1, \ldots, d_{2}\right\},
\end{aligned}
$$

where for $\phi \in C_{c}^{\infty}\left(\mathbf{R}^{d_{1}} ; \mathbf{R}^{d_{2}}\right)$ and $l \in\left\{1, \ldots, d_{2}\right\}$,

$$
\begin{aligned}
\mathcal{N}_{t}^{2 ; l \varrho} \phi(x) & :=\mathbf{1}_{\{2\}}(\alpha) \sigma_{t}^{2 ; i \varrho}(x) \partial_{i} \phi^{l}(x)+v_{t}^{2 ; i \bar{l} \varrho}(x) \phi^{\bar{l}}(x), \varrho \geq 1, \\
\mathcal{I}_{t, z}^{2 ; l} \phi(x) & :=\left(I_{d_{2}}^{l \bar{l}}+\rho_{t}^{2 ; l \bar{l}}(x, z)\right) \phi^{\bar{l}}\left(x+H_{t}^{2}(x, z)\right)-\phi^{l}(x) .
\end{aligned}
$$

By Proposition 3.4, P-a.s. $\Phi\left(\tau, X^{-1}(\tau)\right) \in D\left([0, T] ; C_{l o c}^{\beta^{\prime}}\left(\mathbf{R}^{d_{1}} ; \mathbf{R}^{d_{2}}\right)\right)$ and $\tilde{v}_{t}(\tau, x)=\Phi_{t}\left(\tau, X_{t}^{-1}(\tau, x)\right)$ solves (3.15). We write $v_{t}(x)=v_{t}(\tau, x)$. Moreover, for each $\epsilon>0$ and $p \geq 2$,

$$
\mathbf{E}\left[\sup _{t \leq T}\left|r_{1}^{-\left(\theta \vee \theta^{\prime}\right)-\epsilon} \tilde{v}_{t}(\tau)\right|_{\beta^{\prime}}^{p} \mid \mathcal{F}_{\tau}\right] \leq N\left(\left|r_{1}^{-\theta^{\prime}} \varphi\right|_{\beta^{\prime}}^{p}+1\right),
$$

where $N=N\left(d_{1}, d_{2}, p, N_{0}, T, \beta^{\prime}, \eta^{1}, \eta^{2}, \epsilon, \theta, \theta^{\prime}\right)$ is a positive constant. Without loss of generality we will assume that for all $\omega$ and $t,\left|r_{1}^{-\theta^{\prime}} \varphi\right|_{\beta^{\prime}} \leq N$, since we can always multiply the equation by indicator function. For each $n \in \mathbf{N} \cup\{0\}$, let $C_{l o c}^{n}\left(\mathbf{R}^{d_{1}} ; \mathbf{R}^{d_{2}}\right)$ be the separable 
Fréchet space of $n$-times continuously differentiable functions $f: \mathbf{R}^{d_{1}} \rightarrow \mathbf{R}^{d_{2}}$ endowed with the countable set of semi-norms given by

$$
|f|_{n, \text { int }}=\sum_{0 \leq|\gamma| \leq n} \sup _{|x| \leq k}\left|\partial^{\gamma} f(x)\right|, \quad k \in \mathbf{N} .
$$

Owing to Lemma 4.2, there is a the family of measures $E_{\omega}^{t}(d U),(\omega, t) \in \Omega \times[0, T]$ on $D\left([0, T] ; C_{l o c}^{[\beta]^{-}}\left(\mathbf{R}^{d_{1}} ; \mathbf{R}^{d_{2}}\right)\right)$, corresponding to $\mathfrak{A}=\tilde{v}$ such that for all bounded $G: \Omega \times[0, T] \times$ $[0, T] \times D\left([0, T] ; C_{l o c}^{[\beta]^{-}}\left(\mathbf{R}^{d_{1}} ; \mathbf{R}^{d_{2}}\right)\right) \rightarrow \mathbf{R}^{d_{2}}$ that are $\mathcal{O}_{\mathcal{T}} \times \mathcal{B}([0, T]) \times \mathcal{B}\left(D\left([0, T] ; C_{l o c}^{[\beta]^{-}}\left(\mathbf{R}^{d_{1}} ; \mathbf{R}^{d_{2}}\right)\right)\right)$ measurable, $\mathbf{P}$-a.s. for all $t$, we have

$$
E^{t}\left[G_{t}(t, \tilde{v})\right]=\int_{D\left([0, T] ; C_{l o c}^{\left[\beta^{\prime}\right]^{-}}\left(\mathbf{R}^{\left.\left.d_{1} ; \mathbf{R}^{d_{2}}\right)\right)}\right.\right.} G_{t}(t, U) E^{t}(d U)=\mathbf{E}\left[G_{t}(t, \tilde{v}) \mid \mathcal{F}_{t}\right],
$$

where the right-hand-side is the càdlàg modification of the conditional expectation. Set

$$
\hat{u}_{t}(x)=\hat{u}_{t}(\tau, x)=E^{t}\left[\tilde{v}_{t}(\tau, x)\right]=\int_{D\left([0, T] ; C_{l o c}^{\left[\beta^{\prime}\right]^{-}}\left(\mathbf{R}^{d_{1}} ; \mathbf{R}^{d_{2}}\right)\right)} U_{t}(x) E^{t}(d U) .
$$

Let $\lambda=\left(\theta \vee \theta^{\prime}\right)+\epsilon$. We claim that for all multi-indices $\gamma$ with $|\gamma| \leq[\beta]^{-}, \mathbf{P}$-a.s. for all $t$ and $x$,

$$
\partial^{\gamma}\left[r_{1}^{-\lambda}(x) \hat{u}_{t}(x)\right]=\int_{D\left([0, T] ; C_{l o c}^{\left[\beta^{\prime}\right]^{-}}\left(\mathbf{R}^{\left.\left.d_{1} ; \mathbf{R}^{d_{2}}\right)\right)}\right.\right.} \partial^{\gamma}\left[r_{1}^{-\lambda}(x) U_{t}(x)\right] E^{t}(d U)=E^{t}\left[\partial^{\gamma}\left[r_{1}^{-\lambda}(x) \tilde{v}_{t}(x)\right]\right] .
$$

Indeed, since

$$
M_{t}=E^{t}\left[\sup _{s \leq T}\left|\partial^{\gamma}\left[r_{1}^{-\lambda} \tilde{v}_{s}\right]\right|_{0}\right], \quad t \in[0, T]
$$

is a $(\mathbf{F}, \mathbf{P})$ martingale, we have

$$
\mathbf{E}\left[\sup _{t \leq T}\left|M_{t}\right|^{2}\right] \leq 4 \mathbf{E}\left[\left|M_{T}\right|^{2}\right] \leq 4 \mathbf{E}\left[\sup _{t \leq T}\left|\partial^{\gamma}\left[r_{1}^{-\lambda} \tilde{v}_{t}\right]\right|_{0}^{2} \mid\right]<\infty
$$

and hence $\mathbf{P}$-a.s. for all $t$,

$$
\int_{D\left([0, T] ; C_{l o c}^{\left[\beta^{\prime}\right]^{-}}\right.} \sup _{\left.\left.\mathbf{R}^{d_{1}} ; \mathbf{R}^{d_{2}}\right)\right)}\left|\partial^{\gamma}\left[r_{1}^{-\lambda}(x) U_{s}(x)\right]\right| E^{t}(d U)=E^{t}\left[\sup _{t \leq T}\left|\partial^{\gamma}\left[r_{1}^{-\lambda} \tilde{\mathbf{v}}_{t}\right]\right|_{0}\right]<\infty .
$$

Similarly, since $\mathbf{E}\left[\sup _{t \leq T}\left|r_{1}^{-\lambda} \tilde{v}_{t}\right|_{\beta^{\prime}}^{2}\right]<\infty, \mathbf{P}$-a.s. for each $x$ and $y$,

$$
\begin{aligned}
\frac{\left|\partial^{\gamma}\left[r_{1}^{-\lambda}(x) \hat{u}_{t}(x)\right]-\partial^{\gamma}\left[r_{1}^{-\lambda}(y) \hat{u}_{t}(y)\right]\right|}{|x-y|^{\left\{\beta^{\prime}\right\}^{+}}} & \leq E^{t}\left[\frac{\left|\partial^{\gamma}\left[r_{1}^{-\lambda}(x) \tilde{v}_{t}(x)\right]-\partial^{\gamma}\left[r_{1}^{-\lambda}(y) \tilde{v}_{t}(y)\right]\right|}{|x-y|^{\left\{\beta^{\prime}\right\}^{+}}}\right] \\
& \leq E^{t}\left[\left|r_{1}^{-\lambda} \tilde{v}_{t}\right|_{\beta^{\prime}}\right]
\end{aligned}
$$

and hence, $\mathbf{P}$-a.s.

$$
\sup _{t \leq T}\left|r_{1}^{-\lambda} \hat{u}_{t}\right|_{\beta^{\prime}} \leq \sup _{t \leq T} E^{t}\left[\sup _{t \leq T}\left|r_{1}^{-\lambda} \tilde{v}_{t}\right|_{\beta^{\prime}}\right]<\infty
$$


Thus, P-a.s. $r_{1}^{-\lambda}(\cdot) \hat{u}(\tau) \in D\left([0, T] ; C^{\beta^{\prime}}\left(\mathbf{R}^{d_{1}} ; \mathbf{R}^{d_{2}}\right)\right)$ and (2.4) follows from (3.21) (see the argument (3.22)). For each $l \in\left\{1, \ldots, d_{2}\right\}$, let

$$
\begin{aligned}
\mathcal{A}_{t}^{l}(x)= & \varphi^{l}(x)+\int_{] \tau, \tau \vee t]}\left(\left(\mathcal{L}_{s}^{1 ; l}+\mathcal{L}_{s}^{2 ; l}\right) \hat{u}_{s}(x)+\mathbf{1}_{[1,2]}(\alpha) \hat{b}_{s}^{i}(x) \partial_{i} \hat{u}_{s}^{l}(x)+\hat{c}_{s}^{l \bar{l}}(x) \hat{u}_{s}^{\bar{l}}(x)+\hat{f}_{s}^{l}(x)\right) d s \\
& +\int_{] \tau, \tau \vee t]}\left(\mathcal{N}_{s}^{1 ; l} \hat{u}_{s}(x)+g_{s}^{l \varrho}(x)\right) d w_{s}^{1 ; \varrho} \\
& +\int_{] \tau, \tau \vee t]} \int_{Z^{1}}\left(\mathcal{I}_{s, z}^{1 ; l} \hat{u}_{s-}(x)+h_{s}^{l}(x, z)\right)\left[\mathbf{1}_{D^{1}}(z) q^{1}(d s, d z)+\mathbf{1}_{E^{1}}(z) p^{1}(d s, d z)\right] .
\end{aligned}
$$

By Theorem 12.21 in [Jac79], the representation property holds for (F, P), and hence every bounded $(\mathbf{F}, \mathbf{P})$ - martingale issuing from zero can be represented as

$$
M_{t}=\int_{] 0, t]} o_{s}^{\varrho} d w_{s}^{1 ; \varrho}+\int_{] 0, t]} \int_{Z^{1}} e_{s}(z) q^{1}(d s, d z), \quad t \in[0, T],
$$

where

$$
\mathbf{E} \int_{] 0, T]}\left|o_{s}\right|^{2} d s+\mathbf{E} \int_{] 0, T]} \int_{Z^{1}}\left|e_{s}(z)\right|^{2} \pi^{1}(d z) d s<\infty .
$$

Then for an arbitrary $\mathbf{F}$-stopping time $\bar{\tau} \leq T$ and bounded (F, P)- martingale, applying Itô's product rule and taking the expectation, we obtain

$$
\mathbf{E} \tilde{v}_{\bar{\tau}}(\tau, x) \bar{M}_{\bar{\tau}}=\mathbf{E} \mathcal{A}_{\bar{\tau}}(x) \bar{M}_{\bar{\tau}} .
$$

Since the optional projection is unique, P-a.s. for all $t$ and $x, \hat{u}_{t}(x)=\mathcal{A}_{t}(x)$. This completes the proof.

\subsection{Interlacing a sequence of large jumps (Proof of Theorem 2.5)}

Proof of Theorem 2.5. Fix a stopping time $\tau \leq T$ and random field $\varphi$ such that for some $\beta^{\prime} \in(\alpha, \bar{\beta} \wedge \tilde{\beta})$ and $\theta^{\prime} \geq 0$, P-a.s. $r_{1}^{-\theta^{\prime}} \varphi \in C^{\beta^{\prime}}\left(\mathbf{R}^{d_{1}} ; \mathbf{R}^{d_{2}}\right)$. For any $\delta>0$, we can rewrite (1.1) as

$$
\begin{aligned}
d u_{t}^{l}= & \left(\left(\overline{\mathcal{L}}_{t}^{1 ; l}+\mathcal{L}_{t}^{2 ; l}\right) u_{t}+\mathbf{1}_{[1,2]}(\alpha) \bar{b}_{t}^{i} \partial_{i} u_{t}^{l}+\bar{c}_{t}^{l \bar{l}} u_{t}^{\bar{l}}+f_{t}^{l}\right) d t+\left(\mathcal{N}_{t}^{1 ; l o} u_{t}+g_{t}^{l \varrho}\right) d w_{t}^{1 ; \varrho} \\
& +\int_{Z^{1}}\left(\overline{\mathcal{I}}_{t, z}^{1 ; l} u_{t-}+\bar{h}_{t}^{l}(z)\right)\left[1_{D^{1}}(z) q^{1}(d t, d z)+1_{E^{1}}(z) p^{1}(d t, d z)\right] \\
& +\int_{Z^{1}}\left(\mathbf{1}_{\left(D^{1} \cup E^{1}\right) \cap\left\{K_{t}^{1}>\delta\right\}}(z)+\mathbf{1}_{V^{1}}(z)\right)\left(\mathcal{I}_{t, z}^{1 ; l} u_{t-}+h_{t}^{l}(z)\right) p^{1}(d t, d z), \quad \tau<t \leq T, \\
u_{t}^{l}= & \varphi^{l}, \quad t \leq \tau, \quad l \in\left\{1, \ldots, d_{2}\right\},
\end{aligned}
$$

where for $\phi \in C_{c}^{\infty}\left(\mathbf{R}^{d_{1}} ; \mathbf{R}^{d_{2}}\right)$ and $l \in\left\{1, \ldots, d_{2}\right\}$,

$$
\begin{aligned}
\overline{\mathcal{L}}_{t}^{1 ; l} \phi(x):= & \mathbf{1}_{\{2\}}(\alpha) \frac{1}{2} \sigma_{t}^{1 ; i \varrho}(x) \sigma_{t}^{1 ; j \varrho}(x) \partial_{i j} \phi^{l}(x)+\mathbf{1}_{\{2\}}(\alpha) \sigma_{t}^{k ; i \varrho}(x) v_{t}^{1 ; i \bar{l} \varrho}(x) \partial_{i} \phi^{\bar{l}}(x) \\
& +\int_{D^{1}} \bar{\rho}_{t}^{1 ; i \bar{l}}(x, z)\left(\phi^{\bar{l}}\left(x+\bar{H}_{t}^{1}(x, z)\right)-\phi^{\bar{l}}(x)\right) \pi^{1}(d z) \\
& +\int_{D^{1}}\left(\phi^{l}\left(x+\bar{H}_{t}^{1}(x, z)\right)-\phi^{l}(x)-\mathbf{1}_{(1,2]}(\alpha) \bar{H}_{t}^{1 ; i}(x, z) \partial_{i} \phi^{l}(x)\right) \pi^{1}(d z),
\end{aligned}
$$




$$
\begin{aligned}
\overline{\mathcal{I}}_{t, z}^{1} \phi^{l}(x) & =\left(I_{d_{2}}^{l \bar{l}}+\mathbf{1}_{\left\{K_{t}^{1} \leq \delta\right\}}(z) \rho_{t}^{1 ; l \bar{l}}(x, z)\right) \phi^{\bar{l}}\left(x+\mathbf{1}_{\left\{K_{t}^{1} \leq \delta\right\}}(z) H_{t}^{1}(x, z)\right)-\phi^{l}(x), \\
\bar{H}^{1} & :=\mathbf{1}_{\left\{K_{t}^{1} \leq \delta\right\}} H^{1}, \bar{\rho}^{1}:=\mathbf{1}_{\left\{K_{t}^{1} \leq \delta\right\}} \rho^{1}, \bar{h}:=\mathbf{1}_{\left\{K_{t}^{1} \leq \delta\right\}} h, \\
\bar{b}_{t}^{i}(x) & :=b_{t}^{i}(x)-\int_{D^{1} \cap\left\{K_{t}^{1}>\delta\right\}} \mathbf{1}_{(1,2]}(\alpha) H_{t}^{1 ; i}(x, z) \pi^{1}(d z), \\
\bar{c}_{t}^{l \bar{l}}(x) & :=c_{t}^{l \bar{l}}(x)-\int_{D^{1} \cap\left\{K_{t}^{1}>\delta\right\}} \rho_{t}^{1 ; l \bar{l}}(x, z) \pi^{1}(d z) .
\end{aligned}
$$

For an arbitrary stopping time $\tau^{\prime} \leq T$ and $\mathcal{F}_{\tau^{\prime}} \otimes \mathcal{B}\left(\mathbf{R}^{d_{1}}\right)$-measurable random field $\varphi^{\tau^{\prime}}: \Omega \times$ $\mathbf{R}^{d_{1}} \rightarrow \mathbf{R}^{d_{2}}$ satisfying for some $\theta\left(\tau^{\prime}\right)>0$, P-a.s. $r_{1}^{-\theta\left(\tau^{\prime}\right)} \varphi^{\tau^{\prime}} \in C^{\beta^{\prime}}\left(\mathbf{R}^{d_{1}} ; \mathbf{R}^{d_{2}}\right)$, consider the system of SIDEs on $[0, T] \times \mathbf{R}^{d_{1}}$ given by

$$
\begin{aligned}
d v_{t}^{l}= & \left(\left(\overline{\mathcal{L}}_{t}^{1 ; l}+\mathcal{L}_{t}^{2 ; l}\right) v_{t}+\mathbf{1}_{[1,2]}(\alpha) \bar{b}_{t}^{i} \partial_{i} v_{t}^{l}+\bar{c}_{t}^{l \bar{l}} v_{t}^{\bar{l}}+f_{t}^{l}\right) d t+\left(\mathcal{N}_{t}^{1 ; l \varrho} v_{t}+g_{t}^{l \varrho}\right) d w_{t}^{1 ; \varrho} \\
& +\int_{Z^{1}}\left(\overline{\mathcal{I}}_{t, z}^{1 ; l} u_{t-}+\bar{h}_{t}^{l}(z)\right)\left[1_{D^{1}}(z) q^{1}(d t, d z)+1_{E^{1}}(z) p^{1}(d t, d z)\right], \quad \tau^{\prime}<t \leq T, \\
v_{t}^{l}= & \varphi^{\tau^{\prime} ; l}, \quad t \leq \tau^{\prime}, \quad l \in\left\{1, \ldots, d_{2}\right\} .
\end{aligned}
$$

Set $\bar{H}^{2}=H^{2}$ and $\bar{\rho}^{2}=\rho^{2}$. In order to invoke Theorem 2.2 and obtain a unique solution $v_{t}=v_{t}\left(\tau^{\prime}, x\right)=v_{t}\left(\tau^{\prime}, \varphi^{\tau^{\prime}}, x\right)$ of (3.24), we will show that for all $\omega$ and $t$,

$$
\left|r_{1}^{-1} \tilde{b}_{t}\right|_{0}+\left|\nabla \tilde{b}_{t}\right|_{\bar{\beta}-1}+\left|\tilde{c}_{t}\right|_{\tilde{\beta}}+\left|r^{-\theta} \tilde{f}\right|_{\tilde{\beta}} \leq N_{0}
$$

where

$$
\begin{aligned}
\tilde{b}_{t}^{i}(x):= & \mathbf{1}_{[1,2]}(\alpha) \bar{b}_{t}^{i}(x)-\sum_{k=1}^{2} \mathbf{1}_{\{2\}}(\alpha) \sigma_{t}^{k ; j \varrho}(x) \partial_{j} \sigma_{t}^{k ; \varrho}(x) \\
& -\sum_{k=1}^{2} \int_{D^{k}}\left(\mathbf{1}_{(1,2]}(\alpha) \bar{H}_{t}^{k ; i}(x, z)-\bar{H}_{t}^{k ; i}\left(\tilde{H}_{t}^{k ;-1}(x, z), z\right)\right) \pi^{k}(d z), \\
\tilde{c}_{t}^{l \bar{l}}(x):= & \bar{c}_{t}^{l \bar{l}}(x)-\sum_{k=1}^{2} \mathbf{1}_{\{2\}}(\alpha) \sigma_{t}^{k ; i \varrho}(x) \partial_{i} v_{t}^{k ; l \bar{l} \varrho}(x) \\
& -\sum_{k=1}^{2} \int_{D^{k}}\left(\bar{\rho}_{t}^{k ; \bar{l}}(x, z)-\bar{\rho}_{t}^{k ; l \bar{l}}\left(\tilde{H}_{t}^{k ;-1}(x, z), z\right)\right) \pi^{k}(d z), \\
\tilde{f}_{t}^{l}(x):= & f_{t}^{l}(x)-\sigma_{t}^{1 ; j \varrho}(x) \partial_{j} g_{t}^{l}(x)-\int_{D^{1}}\left(\bar{h}_{t}^{l}(x, z)-\bar{h}_{t}^{l}\left(\tilde{H}_{t}^{1 ;-1}(x, z), z\right)\right) \pi^{1}(d z) .
\end{aligned}
$$

Owing to Assumption $2.3\left(\bar{\beta}, \delta^{1}, \delta^{2}, \mu^{1}, \mu^{2}\right)$, we easily deduce that there is a constant $N=$ $N\left(d_{1}, N_{0}, \bar{\beta}\right)$ such that for each $k \in\{1,2\}$ and all $\omega$ and $t$,

$$
\left|\sigma_{t}^{k ; j \varrho} \partial_{j} \sigma_{t}^{k ; \varrho}\right|_{\bar{\beta}}+\left|\sigma_{t}^{k ; j \varrho} \partial_{j} a_{t}^{k ; \varrho}(x)\right|_{\bar{\beta}}+\left|\sigma_{t}^{1 ; j \varrho} \partial_{j} g_{t}^{\varrho}\right|_{\bar{\beta}} \leq N, \text { if } \alpha=2 .
$$

Since $\left|\nabla \bar{H}_{t}^{1}\right|_{0} \leq \delta$, for any fixed $\eta^{1}<1$, for all $(\omega, t, x, z) \in\left\{(\omega, t, x, z) \in \Omega \times[0, T] \times \mathbf{R}^{d_{1}} \times\right.$ $\left.\left(D^{1} \cup E^{1}\right):\left|\nabla \bar{H}_{t}^{1}(\omega, x, z)\right|>\eta^{1}\right\}$,

$$
\left|\left(I_{d_{1}}+\nabla H_{t}^{1}(\omega, x, z)\right)^{-1}\right| \leq \frac{1}{1-\delta} .
$$


Appealing to Assumption 2.3( $\left.\bar{\beta}, \delta^{1}, \delta^{2}, \mu^{1}, \mu^{2}\right)$ and applying Lemma 4.10, we obtain that there is a constant $N=N\left(d_{1}, d_{2}, N_{0}\right)$ such that for each $k \in\{1,2\}$ and all $\omega, t$, and $z$,

$$
\begin{aligned}
\left|\bar{H}_{t}^{k ; i}(z)-\bar{H}_{t}^{k ; i}\left(\tilde{\bar{H}}_{t}^{k ;-1}(z), z\right)\right|_{\bar{\beta}} \leq & N\left(K_{t}^{k}(z)+\bar{K}_{t}^{k}(z)\right)^{2}+N \mathbf{1}_{(0,1]}\left(\{\bar{\beta}\}^{+}+\delta^{k}\right) \tilde{K}_{t}^{k}(z) K_{t}^{k}(z)^{\delta^{k}} \\
& +N \mathbf{1}_{(1,2]}\left(\{\bar{\beta}\}^{+}+\delta^{k}\right)\left(\tilde{K}_{t}^{k}(z) K_{t}^{k}(z)^{\delta^{k}}+\bar{K}_{t}^{k}(z)^{2}\right), \\
\left|\bar{\rho}_{t}^{k}(z)-\bar{\rho}_{t}^{k}\left(\tilde{\bar{H}}_{t}^{k ;-1}(z), z\right)\right|_{\bar{\beta} \leq} & N l_{t}^{k}(z)\left(K_{t}^{k}(z)+\bar{K}_{t}^{k}(z)\right)+N \mathbf{1}_{(0,1]}\left(\{\bar{\beta}\}^{+}+\mu^{k}\right) \tilde{l}_{t}^{k}(z) K_{t}^{k}(z)^{\mu^{k}} \\
& +N \mathbf{1}_{(1,2]}\left(\{\bar{\beta}\}^{+}+\mu^{k}\right)\left(\tilde{l}_{t}^{k}(z) K_{t}^{k}(z)^{\mu^{k}}+l_{t}^{k}(z) \bar{K}_{t}^{k}(z)\right),
\end{aligned}
$$

and

$$
\begin{aligned}
\left|r_{1}^{-\theta} \bar{h}_{t}(z)-r_{1}^{-\theta} \bar{h}_{t}\left(\tilde{\bar{H}}_{t}^{1 ;-1}(z), z\right)\right|_{\bar{\beta}} \leq & N l_{t}^{1}(z)\left(K_{t}^{1}(z)+\bar{K}_{t}^{k}(z)\right)+N \mathbf{1}_{(0,1]}\left(\{\bar{\beta}\}^{+}+\mu^{1}\right) \tilde{l}_{t}^{k}(z) K_{t}^{1}(z)^{\mu^{1}} \\
& +N \mathbf{1}_{(1,2]}\left(\{\bar{\beta}\}^{+}+\mu^{1}\right)\left(\tilde{l}_{t}^{k}(z) K_{t}^{1}(z)^{\mu^{1}}+l_{t}^{k}(z) \bar{K}_{t}^{1}(z)\right) .
\end{aligned}
$$

Moreover, using Lemma 4.10, we find that there is a constant $N=N\left(d_{1}, d_{2}, N_{0}\right)$ such that for each $k \in\{1,2\}$, and all $\omega, t$, and $z$,

$$
\left|r_{1}^{-1} \bar{H}_{t}^{k}\left(\tilde{\bar{H}}_{t}^{k ;-1}(z), z\right)\right|_{0} \leq\left|r_{1}^{-1} H^{k}\right|_{0}, \quad\left|\nabla\left[\bar{H}_{t}^{k ; i}\left(\tilde{\bar{H}}_{t}^{k ;-1}(z), z\right)\right]\right|_{\bar{\beta}} \leq\left|\nabla H^{k}\right|_{\bar{\beta}-1} .
$$

Combining the above estimates and using Hölder's inequality and the integrability properties of $l_{t}^{k}(z)$ and $K_{t}^{k}(z)$, we obtain (3.25). Therefore, by Theorem 2.2, for each stopping time $\tau^{\prime} \leq T$ and and $\mathcal{F}_{\tau^{\prime}} \otimes \mathcal{B}\left(\mathbf{R}^{d_{1}}\right)$-measurable random field $\varphi^{\tau^{\prime}}$ satisfying for some $\theta\left(\tau^{\prime}\right)>0$, P-a.s. $r_{1}^{-\theta\left(\tau^{\prime}\right)} \varphi^{\tau^{\prime}} \in C^{\beta^{\prime}}\left(\mathbf{R}^{d_{1}} ; \mathbf{R}^{d_{2}}\right)$, there exists a unique solution $v_{t}(x)=v_{t}\left(\tau^{\prime}, \varphi^{\tau^{\prime}}, x\right)$ of (3.24) such that

$$
\mathbf{E}\left[\sup _{t \leq T}\left|r_{1}^{-\theta\left(\tau^{\prime}\right) \vee \theta-\epsilon} v_{t}\left(\tau^{\prime}\right)\right|_{\beta^{\prime}}^{p} \mid \mathcal{F}_{\tau^{\prime}}\right] \leq N\left(\left|r_{1}^{-\theta\left(\tau^{\prime}\right)} \varphi^{\tau^{\prime}}\right|_{\beta^{\prime}}^{p}+1\right)
$$

where $N=N\left(d_{1}, d_{2}, p, N_{0}, T, \beta^{\prime}, \eta^{1}, \eta^{2}, \epsilon, \theta, \theta\left(\tau^{\prime}\right)\right)$ is a positive constant. Let

$$
A_{t}=\int_{] 0, t]} \int_{Z^{1}}\left(\mathbf{1}_{\left(D^{1} \cup E^{1}\right) \cap\left\{K_{s}^{1}>\eta^{1}\right\}}(z)+\mathbf{1}_{V^{1}}(z)\right) p^{1}(d s, d z), \quad t \leq T .
$$

Define a sequence of stopping times $\left(\tau_{n}\right)_{n \geq 0}$ recursively by $\tau_{1}=\tau$ and

$$
\tau_{n+1}=\inf \left(t>\tau_{n}: \Delta A_{t} \neq 0\right) \wedge T
$$

We obtain the existence of a unique solution $u=u(\tau)$ of (3.23) in $\tau^{\beta^{\prime}}\left(\mathbf{R}^{d_{1}} ; \mathbf{R}^{d_{2}}\right)$ by interlacing solutions of (3.24) along the sequence of stopping times $\left(\tau_{n}\right)$. For $(\omega, t) \in\left[\left[0, \tau_{1}\right)\right)$, we set $u_{t}(\tau, x)=v_{t}(\tau, \varphi, x)$ and note that

$$
\mathbf{E}\left[\sup _{t \leq \tau_{1}}\left|r_{1}^{-\theta^{\prime} \vee \theta-\epsilon} u_{t}(\tau)\right|_{\beta^{\prime}}^{p} \mid \mathcal{F}_{\tau}\right] \leq N\left(\left|r_{1}^{-\theta^{\prime}} \varphi\right|_{\beta^{\prime}}^{p}+1\right)
$$

For each $\omega$ and $x$, we set

$$
u_{\tau_{1}}(x)=u_{\tau_{1}-}(x)+\int_{Z^{1}}\left(\mathbf{1}_{\left(D^{1} \cup E^{1}\right) \cap\left\{K^{1}>\eta^{1}\right\}}(t, z)+\mathbf{1}_{V^{1}}(z)\right)\left(\mathcal{I}_{t, z}^{1} u_{\tau_{1}-}(x)+h_{\tau_{1}}^{l}(x, z)\right) p^{1}\left(\left\{\tau_{1}\right\}, d z\right) .
$$


By virtue of Lemma 4.9, there is a constant $N=N\left(d_{1}, d_{2}, \theta, \theta^{\prime}, \zeta_{\tau_{1}}(z), \beta^{\prime}\right)$

$$
\left|u_{\tau_{1}-} \circ \tilde{H}_{\tau_{1}}^{1}(z) \cdot r_{1}^{-\xi_{\tau_{1}}(z)\left(\theta \vee \theta^{\prime}+\epsilon+\beta^{\prime}\right)}\right|_{\beta^{\prime}} \leq N\left|r_{1}^{-\theta \vee \theta^{\prime}-\epsilon} u_{\tau_{1}-}^{l}\right|_{\beta^{\prime}},
$$

and hence

$$
\left|r_{1}^{-\lambda_{1}} u_{\tau_{1}}(x)\right|_{\beta^{\prime}} \leq N\left|r_{1}^{-\theta \vee \theta^{\prime}-\epsilon} u_{\tau_{1}-}^{l}\right|_{\beta^{\prime}}+\zeta_{\tau_{1}}(z)
$$

where

$$
\lambda_{1}=\left(\xi_{\tau_{1}}(z)\left(\theta \vee \theta^{\prime}+1+\epsilon+\beta^{\prime}\right)\right) \vee \theta \vee\left(\theta \vee \theta^{\prime}+\epsilon\right)
$$

We then proceed inductively, each time making use of the estimate (3.26), to obtain a unique solution $u=u(\tau)$ of (3.23), and hence (1.1), in $\widetilde{c}^{\beta^{\prime}}\left(\mathbf{R}^{d_{1}} ; \mathbf{R}^{d_{2}}\right)$. This completes the proof of Theorem 2.5.

\section{Appendix}

\subsection{Martingale and point measure measure moment estimates}

Set $(Z, \mathcal{Z}, \pi)=\left(Z^{1}, \mathcal{Z}^{1}, \pi^{1}\right), p(d t, d z)=p^{1}(d t, d z)$, and $q(d t, d z)=q^{1}(d t, d z)$. We will make use of the following moment estimates to derive the estimates of $\Gamma_{t}$ and $\Psi_{t}$ in Lemma 3.2. The notation $a \underset{p}{\sim} b$ is used to indicate that the quantity $a$ is bounded above and below by a constant depending only on $p$ times $b$.

Lemma 4.1. Let $h: \Omega \times[0, T] \times Z \rightarrow \mathbf{R}^{d_{1}}$ be $\mathcal{P}_{T} \otimes \mathcal{Z}$-measurable

(1) For each stopping time $\tau \leq T$ and $p \geq 2$,

$$
\begin{aligned}
\mathbf{E}\left[\sup _{t \leq \tau}\left|\int_{] 0, t]} \int_{Z} h_{s}(z) q(d s, d z)\right|^{p}\right] \underset{p}{\sim} \mathbf{E}\left[\int_{] 0, \tau]} \int_{Z}\left|h_{s}(z)\right|^{p} \pi(d z) d s\right] \\
+\mathbf{E}\left[\left(\int_{] 0, \tau]} \int_{Z}\left|h_{s}(z)\right|^{2} \pi(d z) d s\right)^{p / 2}\right] .
\end{aligned}
$$

(2) For each stopping time $\tau \leq T$ and $p \geq 1$,

$$
\begin{aligned}
\mathbf{E}\left[\sup _{t \leq \tau}\left(\int_{] 0, t]} \int_{Z}\left|h_{s}(z)\right| p(d s, d z)\right)^{p}\right] \underset{p}{\sim} \mathbf{E}\left[\int_{] 0, \tau]} \int_{Z}\left|h_{s}(z)\right|^{p} \pi(d z) d s\right] \\
+\mathbf{E}\left[\left(\int_{10, \tau]} \int_{Z}\left|h_{s}(z)\right| \pi(d z) d s\right)^{p}\right],
\end{aligned}
$$

Proof. We will only prove part (2), since part (1) is well-known (see, e.g., [Kun04]) and it follows from (2) by the Burkholder-Davis-Gundy inequality. Assume that $h_{t}(\omega, z)>0$ for all $\omega, t$ and $z$. Let

$$
A_{t}=\int_{] 0, t]} \int_{Z} h_{s}(z) p(d s, d z) \quad \text { and } \quad L_{t}=\int_{] 0, t]} \int_{Z} h_{s}(z) \pi(d z) d s, \quad t \leq T .
$$


4.1 Martingale and point measure measure moment estimates

It suffices to prove the claim for $p>1$, since the case $p=1$ is obvious. Fix an arbitrary stopping time $\tau \leq T$ and $p>1$. For all $\omega$ and $t$, we have

$$
A_{t}^{p}=\sum_{s \leq t}\left[\left(A_{s-}+\Delta A_{s}\right)^{p}-A_{s-}^{p}\right]
$$

Thus, using the inequality

$$
b^{p} \leq(a+b)^{p}-a^{p} \leq p(a+b)^{p-1} b \leq p 2^{p-1}\left[a^{p-1} b+b^{p}\right], \quad a, b \geq 0,
$$

for all $\omega$ and $t$, we get

$$
A_{t}^{p} \leq p 2^{p-2}\left[\int_{0}^{t} \int_{Z} A_{s-}^{p-1} h_{s}(z) p(d s, d z)+\int_{] 0, t]} \int_{h} h_{s}(z)^{p} p(d s, d z)\right] .
$$

and

$$
A_{t}^{p} \geq \int_{] 0, t]} \int_{Z} h_{s}(z)^{p} p(d s, d z)
$$

Then since $A_{t}$ is an increasing process, we have

$$
\mathbf{E} \int_{] 0, \tau]} \int_{Z} h_{s}(z)^{p} p(d s, d z) \leq \mathbf{E} A_{\tau}^{p} \leq p 2^{p-2} \mathbf{E}\left[A_{\tau}^{p-1} L_{\tau}+\int_{] 0, \tau]} \int_{Z} h_{s}(z)^{p} p(d s, d z)\right] .
$$

It is easy to see that

$$
\mathbf{E} L_{\tau}^{p}=p \mathbf{E} \int_{] 0, \tau]} L_{s}^{p-1} d L_{s}=p \mathbf{E} \int_{] 0, \tau]} L_{s}^{p-1} d A_{s} \leq p \mathbf{E}\left[L_{\tau}^{p-1} A_{\tau}\right] .
$$

Applying Young's inequality, for all $\varepsilon>0$, P-a.s.,

$$
A_{\tau}^{p-1} L_{\tau} \leq \varepsilon A_{\tau}^{p}+\frac{(p-1)^{p-1}}{\varepsilon^{p-1} p^{p}} L_{\tau}^{p} \quad \text { and } \quad L_{\tau}^{p-1} A_{\tau} \leq \varepsilon L_{\tau}^{p}+\frac{(p-1)^{p-1}}{\varepsilon^{p-1} p^{p}} A_{\tau}^{p} .
$$

Combining the above estimates, for any $\varepsilon_{1} \in\left(0, \frac{1}{p}\right)$, we have

$$
\left(\frac{\varepsilon_{1}^{p-1} p^{p}\left(1-p \varepsilon_{1}\right)}{p(p-1)^{p-1}} \mathbf{E} L_{\tau}^{p}\right) \vee \mathbf{E} \int_{] 0, \tau]} \int_{Z} h_{s}(z)^{p} p(d s, d z) \leq \mathbf{E} A_{\tau}^{p} .
$$

and for any $\varepsilon_{2} \in\left(0, \frac{1}{p 2^{p-2}}\right)$

$$
\mathbf{E} A_{\tau}^{p} \leq \frac{p 2^{p-2}}{\left(1-p 2^{p-2} \varepsilon_{2}\right)} \mathbf{E}\left[\int_{] 0, \tau]} \int_{Z} h_{s}(z)^{p} p(d s, d z)+\frac{(p-1)^{p-1}}{\varepsilon_{2}^{p-1} p^{p}} L_{\tau}^{p}\right],
$$

which completes the proof. 


\subsection{Optional projection}

The following lemma concerning the optional projection plays an integral role in Section 3.4 and the proof of Theorem 2.2.

Lemma 4.2. (cf. Theorem 1 in [Mey76]) Let $\mathcal{X}$ be a Polish space and $D([0, T] ; \mathcal{X})$ be the space of $\mathcal{X}$-valued càdlàg trajectories with the Skorokhod $\mathcal{J}_{1}$-topology. If $\mathfrak{A}$ is a random variable taking values in $D([0, T] ; \mathcal{X})$, then there exists a family of $\mathcal{B}([0, T]) \times \mathcal{F}$-measurable non-negative measures $E^{t}(d U),(\omega, t) \in \Omega \times[0, T]$, on $D([0, T] ; \mathcal{X})$ and a random-variable $\zeta$ satisfying $\mathbf{P}(\zeta<T)=0$ such that $E^{t}(D([0, T] ; \mathcal{X}))=1$ for $t<\zeta$ and $E^{t}(D([0, T] ; \mathcal{X}))=0$ for $t \geq \zeta$. In addition, $E^{t}$ is càdlàg in the topology of weak convergence, $E^{t}=E^{t+}$ for all $t \in[0, T]$, and for each continuous and bounded functional $F$ on $D([0, T] ; \mathcal{X})$, the process $E^{t}(F)$ is the càdlàg version of $\mathbf{E}\left[F(\mathfrak{H}) \mid \mathcal{F}_{t}\right]$. If $G: \Omega \times[0, T] \times[0, T] \times D([0, T] ; \mathcal{X}) \rightarrow \mathbf{R}^{d_{2}}$ is bounded and $\mathcal{O} \times \mathcal{B}([0, T]) \times \mathcal{B}(D([0, T] ; \mathcal{X}))$-measurable, then

$$
\int_{D([0, T] ; X)} G_{t}(\omega, t, U) E^{t}(d U)=E^{t}\left(G_{t}\right)
$$

is the optional projection of $G_{t}(\mathfrak{H})=G_{t}(\omega, t, \mathfrak{A})$. Furthermore, if $G=G_{t}(\omega, t, U)$ is bounded and $\mathcal{P} \times \mathcal{B}([0, T]) \times \mathcal{B}(D([0, T] ; \mathcal{X}))$-measurable, then $E^{t-}\left(G_{t}\right)$ is the predictable projection of $G_{t}(\mathfrak{H})=G_{t}(\omega, t, \mathfrak{A})$.

Proof. We follow the proof of Theorem 1 in [Mey76]. Since $D([0, T] ; \mathcal{X})$ is a Polish space, for each $t \in[0, T]$, there is family of probability measures $\tilde{E}_{\omega}^{t}(d w), \omega \in \Omega$, on $D([0, T] ; \mathcal{X})$ such that for each $A \in \mathcal{B}(D([0, T] ; \mathcal{X})), \tilde{E}^{t}(A)$ is $\mathcal{F}_{t}$-measurable and $\mathbf{P}$-a.s. ,

$$
\mathbf{P}\left(\mathfrak{A} \in A \mid \mathcal{F}_{t}\right)=\tilde{E}^{t}(A) .
$$

For each $\omega \in \Omega$, let $I(\omega)$ be the set of all $t \in(0, T]$ such that for each bounded continuous function $F$ on $D(([0, T] ; \mathcal{X})$, the function

$$
r \mapsto \tilde{E}_{\omega}^{r}(F)=\int_{D([0, T] ; X)} F(w) \tilde{E}^{r}(d w)
$$

has a right-hand limit on $[0, s) \cap \mathbf{Q}$ and a left-hand limit on $(0, s] \cap \mathbf{Q}$ for every rational $s \in[0, T] \cap \mathbf{Q}$. Let $\zeta(\omega)=\sup (t: t \in I(\omega)) \wedge T$. It is easy to see that $\mathbf{P}(\xi<T)=0$. We set $\tilde{E}_{\omega}^{t}=0$ if $\xi(\omega)<t \leq T$. The function $\tilde{E}_{\omega}^{t}$ has left-hand and right-hand limits for all $t \in \mathbf{Q} \cap[0, T]$. We define $E_{\omega}^{t}=\tilde{E}_{\omega}^{t+}$ for each $t \in[0, T)$ (the limit is taken along the rationals), and $E_{\omega}^{T}$ is the left-hand limit at $T$ along the rationals. The statement follows by repeating the proof of Theorem 1 in [Mey76] in an obvious way.

\subsection{Estimates of Hölder continuous functions}

In the coming lemmas, we establish some properties of weighted Hölder spaces that are used Section 3.5 and the proof of Theorem 2.5. 
Lemma 4.3. Let $\beta \in(0,1]$ and $\theta_{1}, \theta_{2} \in \mathbf{R}$ with $\theta_{1}-\theta_{2} \leq \beta$.

(1) There is a constant $c_{1}=c_{1}\left(\theta_{2}, \beta\right)$ such that for all $\phi: \mathbf{R}^{d_{1}} \rightarrow \mathbf{R}$ with $\left|r_{1}^{-\theta_{1}} \phi\right|_{0}+\left[r_{1}^{-\theta_{2}} \phi\right]_{\beta}=$ : $N_{1}<\infty$,

$$
|\phi(x)-\phi(y)| \leq c_{1} N_{1}\left(r_{1}(x)^{\theta_{2}} \vee r_{1}(y)^{\theta_{2}}\right)|x-y|^{\beta},
$$

for all $x, y \in \mathbf{R}^{d_{1}}$.

(2) Conversely, if $\phi: \mathbf{R}^{d_{1}} \rightarrow \mathbf{R}$ satisfies $\left|r_{1}^{-\theta_{1}} \phi\right|_{0}<\infty$ and there is a constant $N_{2}$ such that for all $x, y \in \mathbf{R}^{d_{1}}$,

$$
|\phi(x)-\phi(y)| \leq N_{2}\left(r_{1}(x)^{\theta_{2}} \vee r_{1}(y)^{\theta_{2}}\right)|x-y|^{\beta},
$$

then

$$
\left[r_{1}^{-\theta_{2}} \phi\right]_{\beta} \leq c_{1}\left|r_{1}^{-\theta_{1}} \phi\right|_{0}+N_{2}
$$

Proof. (1) For all $x, y$ with $r_{1}(x)^{\theta_{2}} \geq r_{1}(y)^{\theta_{2}}$, we have

$$
\begin{aligned}
|\phi(x)-\phi(y)| & \leq r_{1}(x)^{\theta_{2}}\left[r_{1}^{-\theta_{2}} \phi\right]_{\beta}|x-y|^{\beta}+r_{1}(y)^{\theta_{1}-\theta_{2}}\left|r_{1}^{-\theta_{1}} \phi\right|_{0}\left|r_{1}^{\theta_{2}}(x)-r_{1}(y)^{\theta_{2}}\right| \\
& \leq\left(\left[r_{1}^{-\theta_{2}} \phi\right]_{\beta}+c_{1}\left|r_{1}^{-\theta} \phi\right|_{0}\right) r_{1}(x)^{\theta_{2}}|x-y|^{\beta},
\end{aligned}
$$

where $c_{1}:=1+\sup _{t \in(0,1)} \frac{1-t^{\theta_{2}}}{(1-t)^{\beta}}$ if $\theta_{2} \geq 0$ and $c_{1}:=1+\sup _{t \in(1, \infty)} \frac{\left(t^{\theta_{2}}-1\right) t^{\beta}}{(t-1)^{\beta}}$ if $\theta_{2}<0$, which proves the first claim. (2) For all $x$ and $y$ with $r_{1}(x)^{\theta_{2}}>r_{1}(y)^{\theta_{2}}$, we have

$$
\begin{gathered}
\left|r_{1}(x)^{-\theta_{2}} \phi(x)-r_{1}(y)^{-\theta_{2}} \phi(y)\right| \\
\leq r_{1}(x)^{-\theta_{2}}|\phi(x)-\phi(y)|+r_{1}(y)^{\theta_{1}-\theta_{2}}\left|r_{1}^{-\theta_{1}}(y) \phi(y)\right|\left|r_{1}(y)^{\theta_{2}} r_{1}(x)^{-\theta_{2}}-1\right| \\
\leq\left(c_{1}\left|r^{-\theta_{1}} \phi\right|_{0}+N_{2}\right)|x-y|^{\beta}
\end{gathered}
$$

which proves the second claim.

Lemma 4.4. Let $\beta, \mu \in(0,1]$ and $\theta_{1}, \theta_{2}, \theta_{3}, \theta_{4} \in \mathbf{R}$ with $\theta_{1}-\theta_{2} \leq \beta, \theta_{3}-\theta_{4} \leq \mu$, and $\theta_{3} \geq 0$. If $\phi: \mathbf{R}^{d_{1}} \rightarrow \mathbf{R}$ and $H: \mathbf{R}^{d_{1}} \rightarrow \mathbf{R}^{d_{1}}$ are such that

$$
\left|r_{1}^{-\theta_{1}} \phi\right|_{0}+\left[r_{1}^{-\theta_{2}} \phi\right]_{\beta}=: N_{1}<\infty \quad \text { and } \quad\left|r_{1}^{-\theta_{3}} H\right|_{0}+\left[r_{1}^{-\theta_{4}} H\right]_{\mu}=: N_{2}<\infty,
$$

then

$$
\left|\phi \circ H \cdot r_{1}^{-\theta_{1} \theta_{3}}\right|_{0} \leq\left|r_{1}^{-\theta_{1}} \phi\right|_{0}\left(1+\left|r_{1}^{-\theta_{3}} H\right|_{0}\right) \leq N_{1}\left(1+N_{2}\right)^{\theta_{1}}
$$

and there is a constant $N=N\left(\beta, \mu, \theta_{1}, \theta_{2}\right)$ such that

$$
\left[\phi \circ H \cdot r_{1}^{-\theta_{2} \theta_{3}-\beta \theta_{4}}\right]_{\beta \mu} \leq N N_{1}\left(1+N_{2}\right)^{\theta_{2}+\beta} .
$$

Proof. For each $x$, we have

$$
r_{1}(H(x)) \leq\left(1+\left|r_{1}^{-\theta_{3}} H\right|_{0}\right) r_{1}(x)^{\theta_{3}} \leq\left(1+N_{2}\right) r_{1}(x)^{\theta_{3}},
$$

and hence

$$
\left|\phi \circ H \cdot r_{1}^{-\theta_{1} \theta_{3}}\right|_{0} \leq\left|r_{1}^{-\theta_{1}} \phi\right|_{0}\left|r_{1}^{\theta_{1}} \circ H \cdot r_{1}^{-\theta_{1} \theta_{3}}\right|_{0} \leq N_{1}\left(1+N_{2}\right)^{\theta_{1}} \text {. }
$$


Using Lemma 4.3, for all $x$ and $y$, we get

$$
\begin{aligned}
|\phi(H(x))-\phi(H(y))| & \leq N N_{1}\left(r_{1}(H(x)) \vee r_{1}(H(y))\right)^{\theta_{2}}|H(x)-H(y)|^{\beta} \\
& \leq N N_{1}\left(1+N_{2}\right)^{\theta_{2}}\left(r_{1}(x) \vee r_{1}(y)\right)^{\theta_{2} \theta_{3}} N_{2}^{\beta}\left(r_{1}(x) \vee r_{1}(y)\right)^{\beta \theta_{4}}|x-y|^{\beta \mu} \\
& \leq N N_{1}\left(1+N_{2}\right)^{\theta_{2}+\beta}\left(r_{1}(x) \vee r_{1}(y)\right)^{\theta_{2} \theta_{3}+\beta \theta_{4}}|x-y|^{\beta \mu},
\end{aligned}
$$

for some constant $N=N\left(\beta, \mu, \theta_{1}, \theta_{2}\right)$. Noting that

$$
\theta_{1} \theta_{3}-\theta_{2} \theta_{3}-\beta \theta_{4}=\left(\theta_{1}-\theta_{2}\right) \theta_{3}-\beta \theta_{4} \leq \beta\left(\theta_{3}-\theta_{4}\right) \leq \beta \mu,
$$

we apply Lemma 4.3 to complete the proof.

Remark 4.5. Let $\beta \in(0,1]$ and $\theta_{1}, \theta_{2} \in \mathbf{R}$. Then there is a constant $N=N\left(\beta, \theta_{1}, \theta_{2}\right)$ such that for all $\phi: \mathbf{R}^{d_{1}} \rightarrow \mathbf{R}$ with $\left|r_{1}^{-\theta_{1}} \phi\right|_{0}+\left[r_{1}^{-\theta_{2}} \phi\right]_{\beta}=: N_{1}<\infty$, we have $\left|r^{-\theta} \phi\right|_{\beta} \leq N N_{1}$, where $\theta=\max \left\{\theta_{1}, \theta_{2}\right\}$. In particular, if in Lemma 4.4, $\theta_{1}=\theta_{2}$ and $\theta_{4} \geq 0$, then

$$
\left|\phi \circ H \cdot r^{-\theta_{1} \theta_{3}-\beta \theta_{4}}\right|_{\beta \mu} \leq N N_{1}\left(1+N_{2}\right)^{\theta_{1}+\beta} \text {. }
$$

Proof. If $\theta_{2} \geq \theta_{1}$, then the claim is obvious and if $\theta_{1}>\theta_{2}$, for all $x$ and $y$, we have

$$
\begin{aligned}
\left|r_{1}(x)^{-\theta_{1}} \phi(x)-r_{1}(y)^{-\theta_{1}} \phi(y)\right| \leq & r_{1}(x)^{\theta_{2}-\theta_{1}}\left|r_{1}(x)^{-\theta_{2}} \phi(x)-r_{1}(y)^{-\theta_{2}} \phi(y)\right| \\
& +\left|\frac{r(y)^{\theta_{1}-\theta_{2}}}{r(x)^{\theta_{1}-\theta_{2}}}-1\right|\left|r_{1}^{-\theta_{1}} \phi\right|_{0} \leq N_{1}\left(1+c_{1}\right)|x-y|^{\beta},
\end{aligned}
$$

where $c_{1}:=\sup _{t \in(0,1)} \frac{1-t^{\theta_{1}-\theta_{2}}}{(1-t)^{\beta}}$.

Lemma 4.6. For each $\theta \geq 0$ and $\beta>1$, there are constants $N_{1}=N_{1}\left(d_{1}, \theta, \beta\right)$ and $N_{2}\left(d_{1}, \theta, \beta\right)$ such that for all $\phi: \mathbf{R}^{d_{1}} \rightarrow \mathbf{R}$,

$$
N_{1}\left|r_{1}^{-\theta} \phi\right|_{\beta} \leq \sum_{|\gamma| \leq[\beta]^{-}}\left|r_{1}^{-\theta} \partial^{\gamma} \phi\right|_{0}+\sum_{|\gamma|=[\beta]^{-}}\left|r_{1}^{-\theta} \partial^{\gamma} \phi\right|_{\{\beta\}^{+}} \leq N_{2}\left|r_{1}^{-\theta} \phi\right|_{\beta}
$$

Proof. For each multi-index $\gamma$ with $|\gamma| \leq[\beta]^{-}$and $x$, we have

$$
\partial^{\gamma}\left(r_{1}^{-\theta} \phi\right)(x)=\sum_{\substack{\gamma_{1}+\gamma_{2}+=\gamma \\\left|\gamma_{1}\right| \geq 1}} r_{1}(x)^{\theta} \partial^{\gamma_{1}}\left(r_{1}^{-\theta}\right)(x) r_{1}(x)^{-\theta} \partial^{\gamma_{2}} \phi(x)+r_{1}(x)^{-\theta} \partial^{\gamma} \phi(x) .
$$

It is easy to show by induction that for all multi-indices $\gamma,\left|r_{1}^{\theta} \partial^{\gamma}\left(r_{1}^{-\theta}\right)\right|_{1}<\infty$. Moreover, for all multi-indices $\gamma$ with $|\gamma|<[\beta]^{-}$,

$$
\left|r_{1}^{-\theta} \partial^{\gamma} \phi\right|_{1} \leq\left|\nabla\left(r_{1}^{-\theta} \partial^{\gamma} \phi\right)\right| \leq\left|r_{1}^{-\theta} \nabla\left(r_{1}^{-\theta}\right)\right|_{0}\left|r_{1}^{-\theta} \partial^{\gamma} \nabla \phi\right|_{0} .
$$

Thus, for each multi-index $\gamma$ with $|\gamma| \leq[\beta]^{-}$,

$$
\left|\partial^{\gamma}\left(r_{1}^{-\theta} \phi\right)\right|_{0} \leq \sum_{\substack{\gamma_{1}+\gamma_{2}+=\gamma \\ \gamma_{1} \mid \geq 1}}\left|r_{1}^{\theta} \partial^{\gamma_{1}}\left(r_{1}^{-\theta}\right)\right|_{0}\left|r_{1}^{-\theta} \partial^{\gamma_{2}} \phi\right|_{0}+\left|r_{1}^{-\theta} \partial^{\gamma} \phi\right|_{0}
$$


and for each multi-index $\gamma$ with $|\gamma|=[\beta]^{-}$,

$$
\left|\partial^{\gamma}\left(r_{1}^{-\theta} \phi\right)\right|_{\{\beta\}^{+}} \leq \sum_{\substack{\gamma_{1}+\gamma_{2}+=\gamma \\ \gamma_{1} \mid \geq 1}}\left|r_{1}^{\theta} \partial^{\gamma_{1}}\left(r_{1}^{-\theta}\right)\right|_{1}\left|r_{1}^{-\theta} \nabla\left(r_{1}^{-\theta}\right)\right|_{0}\left|r_{1}^{-\theta} \partial^{\gamma_{2}} \nabla \phi\right|_{0}+\left|r_{1}^{-\theta} \partial^{\gamma} \phi\right|_{0} .
$$

This proves the leftmost inequality in (4.1). For all $i \in\{1, \ldots, d\}$ and $x$,

$$
r_{1}^{-\theta} \partial_{i} \phi(x)=\partial_{i}\left(r_{1}^{-\theta} \phi\right)(x)-r_{1}(x)^{-\theta} \phi(x) r_{1}(x)^{\theta} \partial_{i}\left(r_{1}^{-\theta}\right)(x) .
$$

It follows by induction that for all multi-indices $\gamma$ with $|\gamma| \leq[\beta]^{-}$and $x, r_{1}^{-\theta} \partial^{\gamma} \phi(x)$ is a sum of $\partial^{\gamma}\left(r_{1}^{-\theta} \phi\right)(x)$, a finite sum of terms, each of which is a product of one term of the form $\partial^{\tilde{\gamma}}\left(r_{1}^{-\theta} \phi\right)(x),|\tilde{\gamma}|<|\gamma|$, and a finite number of terms of the form $\partial^{\gamma_{1}}\left(r_{1}^{\theta}\right) \partial^{\gamma_{2}}\left(r_{1}^{-\theta}\right),\left|\gamma_{1}\right|,\left|\gamma_{2}\right| \leq|\gamma|$. Since for all multi-indices $\gamma_{1}$ and $\gamma_{2}$, we have $\left|\partial^{\gamma_{1}}\left(r_{1}^{\theta}\right) \partial^{\gamma_{2}}\left(r_{1}^{-\theta}\right)\right|_{1}<\infty$, the rightmost inequality in (4.1) follows.

Corollary 4.7. For each $\theta \geq 0$ and $\beta>1$, there are constants $N_{1}=N_{1}\left(d_{1}, \theta, \beta\right)$ and $N_{2}\left(d_{1}, \theta, \beta\right)$ such that for all $\phi: \mathbf{R}^{d_{1}} \rightarrow \mathbf{R}$,

$$
N_{1}\left|r_{1}^{-\theta} \phi\right|_{\beta} \leq\left|r_{1}^{-\theta} \phi\right|_{0}+\sum_{|\gamma|=[\beta]^{-}}\left|r_{1}^{-\theta} \partial^{\gamma} \phi\right|_{\{\beta\}^{+}} \leq N_{2}\left|r_{1}^{-\theta} \phi\right|_{\beta}
$$

Proof. It is well known that for an arbitrary unit ball $B \subset \mathbf{R}^{d_{1}}$ and any $1 \leq k<[\beta]^{-}$, there is a constant $N$ such that for any $\varepsilon>0$,

$$
\sup _{x \in B,|\gamma|=k}\left|\partial^{\gamma} \phi\right| \leq N\left(\varepsilon \sup _{x \in B,|\gamma|=[\beta]^{-}}\left|\partial^{\gamma} \phi(x)\right|+\varepsilon^{-k} \sup _{x \in B}|\phi(x)|\right) .
$$

Let $U_{0}=\left\{x \in \mathbf{R}^{d_{1}}:|x| \leq 1\right\}$ and $U_{j}=\left\{x \in \mathbf{R}^{d_{1}}: 2^{j-1} \leq|x| \leq 2^{j}\right\}, j \geq 1$. For each $j$, we have

$$
\begin{aligned}
\sup _{x \in U_{j},|\gamma|=k}\left|\partial^{\gamma} \phi(x)\right| & =\sup _{B \subseteq U_{j}} \sup _{x \in B,|\gamma|=k}\left|\partial^{\gamma} \phi(x)\right| \leq N\left(\varepsilon \sup _{B \subseteq U_{j}} \sup _{x \in B,|\gamma|=[\beta]^{-}}\left|\partial^{\gamma} \phi(x)\right|+\varepsilon^{-k} \sup _{B \subseteq U_{j}} \sup _{x \in B}|\phi(x)|\right) \\
& \leq N\left(\varepsilon \sup _{x \in U_{j},|\gamma|=[\beta]^{-}}\left|\partial^{\gamma} \phi(x)\right|+\varepsilon^{-k} \sup _{x \in U_{j}}|\phi(x)|\right) .
\end{aligned}
$$

Since for every $j$,

$$
2^{-\theta / 2} 2^{-j \theta} \sup _{x \in U_{j},|\gamma|=k}\left|\partial^{\gamma} \phi(x)\right| \leq \sup _{x \in U_{j},|\gamma|=k}\left|r^{-\theta} \partial^{\gamma} \phi(x)\right| \leq 2^{\theta} 2^{-(j-1) \theta} \sup _{x \in U_{j},|\gamma|=k}\left|\partial^{\gamma} \phi(x)\right|,
$$

we see that

$$
\begin{aligned}
2^{-\theta / 2} \sup _{j} 2^{-j \theta} \sup _{x \in U_{j},|\gamma|=k}\left|\partial^{\gamma} \phi(x)\right| & \leq \sup _{j} \sup _{x \in U_{j},|\gamma|=k}\left|r^{-\theta} \partial^{\gamma} \phi(x)\right|=\left|r^{-\theta} \partial^{\gamma} \phi\right|_{0} \\
& \leq 2^{\theta} \sup _{j} 2^{-j \theta} \sup _{x \in U_{j},|\gamma|=k}\left|\partial^{\gamma} \phi(x)\right|,
\end{aligned}
$$

and the statement follows. 
Remark 4.8. If $\phi: \mathbf{R}^{d_{1}} \rightarrow \mathbf{R}$ is such that $\left|r^{-\theta_{1}} \phi\right|_{0}+\left|r^{-\theta_{2}} \nabla \phi\right|_{0}<\infty$ for $\theta_{1}, \theta_{2} \in \mathbf{R}$ with $\theta_{1}-\theta_{2} \leq 1$, then

$$
\left[r^{-\theta_{2}} \phi\right]_{1} \leq N\left(\left|r^{-\theta_{1}} \phi\right|_{0}+\left|r^{-\theta_{2}} \nabla \phi\right|_{0}\right)
$$

Proof. Indeed, for each $x$ and $y$, we have

$$
|\phi(x)-\phi(y)| \leq\left|r^{-\theta_{2}} \nabla \phi\right|_{0} \int_{0}^{1} r^{\theta_{2}}(x+s(y-x)) d s|y-x| \leq\left|r^{-\theta_{2}} \nabla \phi\right|_{0}\left(r(y)^{\theta_{2}} \vee r(x)^{\theta_{2}}\right)|y-x|,
$$

and hence the claim follows from Lemma 4.3.

Lemma 4.9. Let $n \in \mathbf{N}, \beta, \mu \in(0,1], \theta_{3}, \theta_{4} \geq 0$ be such that $\theta_{3}-\theta_{4} \leq 1$. There is a constant $N=N\left(d_{1}, \theta_{1}, \theta_{3}, \theta_{4}, n, \beta\right)$ such that for all $\phi: \mathbf{R}^{d_{1}} \rightarrow \mathbf{R}$ with $r_{1}^{-\theta_{1}} \phi \in C^{n+\beta}\left(\mathbf{R}^{d_{1}}, \mathbf{R}^{d_{1}}\right)$ and $H: \mathbf{R}^{d_{1}} \rightarrow \mathbf{R}^{d_{1}}$ with

$$
\left|r_{1}^{-\theta_{3}} H\right|_{0}+\left|r_{1}^{-\theta_{4}} \nabla H\right|_{n-1+\mu}=: N_{2}<\infty
$$

we have

$$
\left|\phi \circ H \cdot r^{-\theta_{1} \theta_{3}}\right|_{0} \leq\left|r_{1}^{-\theta_{1}} \phi\right|_{0}\left(1+\left|r_{1}^{-\theta_{3}} H\right|_{0}\right)^{\theta_{1}}
$$

and

$$
\left|r_{1}^{-\theta_{1} \theta_{3}-\theta_{4}(n+\mu \wedge \beta)} \nabla(\phi \circ H)\right|_{n-1+\mu \wedge \beta} \leq N\left|r_{1}^{-\theta_{1}} \phi\right|_{n+\beta}\left(1+N_{2}\right)^{\theta_{1}+\mu \wedge \beta+n} .
$$

Proof. It follows immediately from Lemma 4.4 and Remark 4.8 that

$$
\left|\phi \circ H \cdot r^{-\theta_{1} \theta_{3}}\right|_{0} \leq\left|r_{1}^{-\theta_{1}} \phi\right|_{0}\left(1+\left|r_{1}^{-\theta_{3}} H\right|_{0}\right)^{\theta_{1}} .
$$

Using induction, we get that for each $x$ and $|\gamma|=n$,

$$
\partial^{\gamma}(\phi(H(x)))=\mathcal{I}_{1}^{\gamma}(x)+\mathcal{I}_{2}^{\gamma}(x)+I_{3}^{\gamma}(x)
$$

where

$$
\mathcal{I}_{1}^{\gamma}(x)=\sum_{i=1}^{d_{1}} \partial_{i} \phi(H(x)) \partial^{\gamma} H^{i}(x)
$$

$\mathcal{I}_{2}^{\gamma}(x)$ is a finite sum of terms of the form

$$
\partial_{i_{1}} \cdots \partial_{i_{|\gamma|}} \phi(H(x)) \partial^{\tilde{\gamma}_{1}} H^{i_{1}} \cdots \partial^{\tilde{\gamma}_{|\gamma|}} H^{i_{|\gamma|}}
$$

with $i_{1}, \ldots, i_{|\gamma|} \in\{1,2, \ldots, d\},\left|\tilde{\gamma}_{1}\right|=\cdots=\left|\tilde{\gamma}_{|\gamma|}\right|=1$, and $\sum_{k=1}^{|\gamma|} \tilde{\gamma}_{k}=\gamma$, if $n \geq 2$ and zero otherwise, and where $\mathcal{I}_{3}^{\gamma}(x)$ is a finite sum of terms of the form

$$
\partial_{i_{1}} \cdots \partial_{i_{k}} \phi(H(x)) \partial^{\tilde{\gamma}_{1}} H^{i_{1}}(x) \cdots \partial^{\tilde{\gamma}_{k}} H^{i_{k}}(x)
$$

with $2 \leq k<n, i_{1}, i_{2}, \ldots, i_{k} \in\{1, \ldots, d\}$, and $\sum_{j=1}^{k} \tilde{\gamma}_{j}=\gamma, 1 \leq\left|\tilde{\gamma}_{j}\right|<|\gamma|$, if $n \geq 3$, and zero otherwise. Thus, owing to Lemmas 4.4 and 4.6, for any multi-index $\gamma$ with $|\gamma|=n$, we have

$$
\left|r_{1}^{-\theta_{3} \theta_{1}-\theta_{4}} \mathcal{I}_{1}^{\gamma}\right|_{0} \leq N\left|r_{1}^{-\theta_{1}} \nabla \phi\right|_{0}\left(1+\left|r_{1}^{-\theta_{3}} H\right|_{0}\right)^{\theta_{1}}\left|r_{1}^{-\theta_{4}} \partial^{\gamma} H\right|_{0}
$$




$$
\left|r_{1}^{-\theta_{3} \theta_{1}-n \theta_{4}} \mathcal{I}_{2}^{\gamma}\right|_{0} \leq N\left|r_{1}^{-\theta_{1}} \partial^{\gamma} \phi\right|_{0}\left(1+\left|r_{1}^{-\theta_{3}} H\right|_{0}\right)^{\theta_{1}}\left|r_{1}^{-\theta_{4}} \nabla H\right|_{0}^{n},
$$

and

$$
\left|r_{1}^{-\theta_{3} \theta_{1}-(n-1) \theta_{4}} \mathcal{I}_{3}^{\gamma}\right|_{0} \leq N\left|r_{1}^{-\theta_{1}} \phi\right|_{n-1}\left(1+\left|r_{1}^{-\theta_{3}} H\right|_{0}+\left|r_{1}^{-\theta_{4}} \nabla H\right|\right)^{\theta_{1}+n-1},
$$

and hence

$$
\left|r^{-\theta_{1} \theta_{3}-n \theta_{4}} \partial^{\gamma}(\phi \circ H)\right|_{0} \leq N\left|r_{1}^{-\theta_{1}} \phi\right|_{n}\left(1+\left|r_{1}^{-\theta_{3}} H\right|_{0}+\left|r_{1}^{-\theta_{4}} \nabla H\right|\right)^{\theta_{1}+n} .
$$

Once again appealing to Lemmas 4.4 and 4.6, for all multi-indices $\gamma$ with $|\gamma|=n$, we get

$$
\begin{gathered}
\left|r_{1}^{-\theta_{1} \theta_{3}-(1+\mu \wedge \beta) \theta_{4}} \mathcal{I}_{1}^{\gamma}\right|_{\mu \wedge \beta} \leq N\left|r_{1}^{-\theta_{1}} \phi\right|_{1+\mu \wedge \beta}\left(1+N_{2}\right)^{\theta_{1}+\mu \wedge \beta+1}, \\
\left|r_{1}^{-\theta_{1} \theta_{3}-(n+\mu \wedge \beta) \theta_{4}} \mathcal{I}_{2}^{\gamma}\right|_{\mu \wedge \beta}+\left|r_{1}^{-\theta_{1} \theta_{3}-(n-1+\mu \wedge \beta) \theta_{4}} \mathcal{I}_{3}^{\gamma}\right|_{\mu \wedge \beta} \leq N\left|r_{1}^{-\theta_{1}} \phi\right|_{n+\mu \wedge \beta}\left(1+N_{2}\right)^{\theta_{1}+n+\mu \wedge \beta} .
\end{gathered}
$$

Then applying Lemmas 4.4 and 4.6, we complete the proof.

We shall now provide some useful estimates of composite functions of diffeomorphisms. Lemma 4.10. Let $H: \mathbf{R}^{d_{1}} \rightarrow \mathbf{R}^{d_{1}}$ be continuously differentiable and assume that for all $x \in \mathbf{R}^{d_{1}}$,

$$
|H(x)| \leq L_{0}+L_{1}|x| \quad \text { and } \quad|\nabla H(x)| \leq L_{2} .
$$

Assume that for all $x \in \mathbf{R}^{d_{1}}, \kappa(x)=\left(I_{d_{1}}+\nabla H(x)\right)^{-1}$ exists and $|\kappa(x)| \leq N_{\kappa}$.

(1) Then the mapping $\tilde{H}(x):=x+H(x)$ is a diffeomorphism with $\tilde{H}^{-1}(x)=x-H\left(\tilde{H}^{-1}(x)\right)=$ : $x+F(x)$ and for all $x \in \mathbf{R}^{d_{1}}$,

$$
|F(x)| \leq L_{0}+L_{1} L_{0} N_{\kappa}+L_{1} N_{\kappa}|x|, \quad|\nabla F(x)| \leq N_{\kappa} L_{2}, \quad\left|\left(I_{d_{1}}+\nabla F(x)\right)^{-1}\right| \leq 1+L_{2} .
$$

For all $p \in \mathbf{R}$, there is a constant $N=N\left(L_{0}, L_{1}, N_{\kappa}, p\right)$ such that for all $x \in \mathbf{R}^{d_{1}}$,

$$
\frac{r_{1}^{p}(\tilde{H}(x))}{r_{1}^{p}(x)}+\frac{r_{1}^{p}\left(\tilde{H}^{-1}(x)\right)}{r_{1}^{p}(x)} \leq N, \quad r_{1}^{-1}(x)\left|H^{i}(x)+F^{k ; i}(x)\right| \leq N[H]_{1}\left|r_{1}^{-1} H\right|_{0} .
$$

Moreover, there is a constant $N=N\left(L_{0}, L_{1}, N_{\kappa}, p\right)$ such that

$$
\begin{gathered}
\left|\frac{r_{1}^{p}(\tilde{H})}{r_{1}^{p}}-1+\mathbf{1}_{(1,2]}(\alpha) p H^{i} r_{1}^{-2} x^{i}\right|_{\alpha}+\left|\frac{r_{1}^{p}\left(\tilde{H}^{-1}\right)}{r_{1}^{p}}-1-\mathbf{1}_{(1,2]}(\alpha) p F^{i} r_{1}^{-2} x^{i}\right|_{\alpha} \\
\leq N\left(\left|r_{1}^{-1} H\right|_{0}^{[\alpha]^{-}+1}+[H]_{1}^{[\alpha]^{-}+1}\right) .
\end{gathered}
$$

(2) If for some $\beta>1,|\nabla H|_{\beta-1} \leq L_{3}$, then there is a constant $N=N\left(d_{1}, \beta, N_{\kappa}, L_{3}\right)$ such that

$$
|\nabla F|_{\beta-1} \leq N|\nabla H|_{\beta-1}
$$

(3) If for some $\beta \geq 1,|\nabla H|_{\beta-1} \leq L_{3}$, then for each $\theta \geq 0$, there is a constant $N=N\left(d_{1}, \beta, N_{\kappa}\right.$, $\left.L_{1}, L_{3}, \theta\right)$ such that

$$
\left|\frac{r_{1}^{\theta} \circ \tilde{H}^{-1}}{r_{1}^{\theta}}-1\right|_{\beta} \leq N\left[\left|r_{1}^{-1} H\right|_{0}+|\nabla H|_{\beta-1}\right]
$$


(4) If $|H|_{0} \leq L_{4}$, and for some $\beta>0,|\nabla H|_{\beta \vee 1-1} \leq L_{5}$ and $\phi: \mathbf{R}^{d_{1}} \rightarrow \mathbf{R}$ is such that for some $\mu \in(0,1]$ and $\theta \geq 0, r_{1}^{-\theta} \phi \in C^{\beta+\mu}\left(\mathbf{R}^{d_{1}} ; \mathbf{R}\right)$, then there is a constant $N=$ $N\left(d_{1}, \beta, \mu, N_{\kappa}, L_{4}, L_{5}, \theta\right)$ such that

$$
\begin{aligned}
\left|r_{1}^{-\theta}\left(\phi \circ \tilde{H}^{-1}-\phi\right)\right|_{\beta} \leq & N\left|r_{1}^{-\theta} \phi\right|_{\beta}\left(|H|_{0}+|\nabla H|_{\beta \vee 1-1}\right) \\
& +N \mathbf{1}_{(0,1]}\left(\{\beta\}^{+}+\mu\right) \sum_{|\gamma|=[\beta]^{-}}\left[\partial^{\gamma}\left(r_{1}^{-\theta} \phi\right)\right]_{\{\beta\}^{+}+\mu} L_{4}^{\mu} \\
& +N \mathbf{1}_{(1,2]}\left(\{\beta\}^{+}+\mu\right) \sum_{|\gamma|=[\beta]^{-}}\left(\left[\nabla \partial^{\gamma}\left(r_{1}^{-\theta} \phi\right)\right]_{\{\beta\}^{+}+\mu-1} L_{4}^{\mu}+\left|\nabla \partial^{\gamma}\left(r_{1}^{-\theta} \phi\right)\right|_{0}|\nabla H|_{0}\right) .
\end{aligned}
$$

Proof. (1) Since $\left(I_{d_{1}}+\nabla H(x)\right)^{-1}$ exists for each $x$, it follows from Theorem 0.2 in [DHI13] that the mapping $\tilde{H}$ is a global diffeomorphism. For each $x$, we easily verify $\tilde{H}^{-1}(x)=$ $x-H\left(\tilde{H}^{-1}(x)\right)$ by substituting $\tilde{H}(x)$ into the expression. Simple computations show that for all $x$, we have

$|\nabla \tilde{H}(x)| \leq 1+L_{2}, \quad\left|\nabla \tilde{H}^{-1}(x)\right|=\left|\kappa\left(\tilde{H}^{-1}(x)\right)\right| \leq N_{\kappa}, \quad|\nabla F(x)|=\left|\nabla H\left(\tilde{H}^{-1}(x)\right) \nabla \tilde{H}^{-1}(x)\right| \leq N_{\kappa} L_{2}$, and

$$
\left|\left(I_{d_{1}}+\nabla F(x)\right)^{-1}\right|=\left|\nabla \tilde{H}^{-1}(x)^{-1}\right|=\left|\kappa\left(\tilde{H}^{-1}(x)\right)^{-1}\right|=\left|I_{d_{1}}+\nabla H\left(\tilde{H}^{-1}(x)\right)\right| \leq 1+L_{2} .
$$

For all $x$ and $y$, we easily obtain

$$
|\tilde{H}(x)-\tilde{H}(y)| \leq\left(1+L_{2}\right)|x-y|, \quad\left|\tilde{H}^{-1}(x)-\tilde{H}^{-1}(y)\right| \leq N_{\kappa}|x-y|,
$$

and hence

$$
N_{\kappa}^{-1}|x-y| \leq|\tilde{H}(x)-\tilde{H}(y)|, \quad\left(1+L_{2}\right)^{-1}|x-y| \leq\left|\tilde{H}^{-1}(x)-\tilde{H}^{-1}(y)\right| .
$$

Making use of (4.3), for all $x$, we get

$$
N_{\kappa}^{-1}|x| \leq L_{0}+|\tilde{H}(x)|, \quad\left|\tilde{H}^{-1}(x)\right| \leq N_{\kappa} L_{0}+N_{\kappa}|x|, \quad|x| \leq L_{0}+L_{1}\left|\tilde{H}^{-1}(x)\right|,
$$

and thus

$$
|F(x)| \leq L_{0}+L_{1} N_{\kappa} L_{0}+L_{1} N_{\kappa}|x| .
$$

The rest of the estimates then follow easily from the above estimates and Taylor's theorem. (2) Using the chain rule, for all $x$, we obtain

$$
\nabla F(x)=-\nabla H\left(\tilde{H}^{-1}(x)\right) \nabla \tilde{H}^{-1}(x)=-\nabla H\left(\tilde{H}^{-1}(x)\right) \kappa\left(\tilde{H}^{-1}(x)\right),
$$

and hence $|\nabla F|_{0} \leq N_{\kappa}|\nabla H|_{0}$. For all $x$ and $y$, we have

$$
\kappa\left(\tilde{H}^{-1}(y)\right)-\kappa\left(\tilde{H}^{-1}(x)\right)=\kappa(y)\left[\nabla H\left(\tilde{H}^{-1}(x)\right)-\nabla H\left(\tilde{H}^{-1}(y)\right)\right] \kappa(x),
$$


and thus since $\left[\tilde{H}^{-1}\right]_{1} \leq\left(1+N_{\kappa} L_{3}\right)$ by part (1), we have for all $\delta \in(0,1 \wedge \beta]$,

$$
\left[\kappa\left(\tilde{H}^{-1}\right)\right]_{\delta} \leq N_{\kappa}^{2}\left(1+N_{\kappa} L_{3}\right)^{\delta}[\nabla H]_{\delta} .
$$

It follows that there is a constant $N=N\left(N_{\kappa}, L_{3}\right)$ such that for all $\delta \in(0,1 \wedge \beta]$,

$$
|\nabla F|_{\delta} \leq N|H|_{\delta}
$$

It is well-known that the inverse map $\mathfrak{J}$ on the set of invertible $d_{1} \times d_{1}$ matrices is infinitely differentiable and for each $n$, there exists a constant $N=N\left(n, d_{1}\right)$ such that for all invertible matrices $A$, the $n$-th derivative of $\mathfrak{I}$ evaluated at $A$, denoted $\mathfrak{I}^{(n)}(A)$, satisfies

$$
\left|\mathfrak{J}^{(n)}(A)\right| \leq N\left|A^{-n-1}\right| \leq N\left|A^{-1}\right|^{n+1} .
$$

Using induction, we find that for all multi-indices $\gamma$ with $|\gamma| \leq[\beta]^{-}$and for each $x, \partial^{\gamma} F(x)$ is a finite sum of terms, each of which is a finite product of

$$
\partial^{\bar{\gamma}} H\left(\tilde{H}^{-1}(x)\right), \quad \kappa\left(\tilde{H}^{-1}(x)\right)^{\bar{n}}, \quad \text { and } \quad \mathfrak{J}^{(\bar{n}-1)}\left(I+\nabla H\left(\tilde{H}^{-1}(x)\right)\right), \quad|\bar{\gamma}| \leq|\gamma|, \quad \bar{n} \in\{1, \ldots,|\gamma|\} .
$$

Therefore, differentiating (4.4) and estimating directly, we easily obtain (4.2).

(3) For each $x$, we have

$$
\begin{aligned}
\frac{r_{1}\left(\tilde{H}^{-1}(x)\right)^{\theta}}{r_{1}(x)^{\theta}}-1 & =r_{1}(x)^{-\theta} \int_{0}^{1} r_{1}\left(G_{s}(x)\right)^{\theta-2} G_{s}(x)^{*} F(x) d s \\
& =\int_{0}^{1} \frac{r_{1}^{\theta-1}\left(G_{s}(x)\right)}{r_{1}(x)^{\theta-1}} K\left(G_{s}(x)\right)^{*} d s r_{1}(x)^{-1} F(x),
\end{aligned}
$$

where $G_{s}(x):=x+s F(x), s \in[0,1]$, and $J(x):=r_{1}(x)^{-1} x$. According to part (1) and (2), we have $\left|r_{1}^{-1} F\right|_{0} \leq N\left|r_{1}^{-1} H\right|_{0}$ and $|\nabla F|_{\beta-1} \leq N|\nabla H|_{\beta-1}$, and hence

$$
\left|r_{1}^{-1} G_{s}\right|_{0} \leq N\left(1+\left|r_{1}^{-1} H\right|_{0}\right), \quad\left|\nabla G_{s}(x)\right|_{\beta-1} \leq N\left(1+|\nabla H|_{\beta-1}\right) .
$$

and

$$
\left|J \circ G_{s}\right|_{\beta} \leq N\left(1+\left|r_{1}^{-1} H\right|_{0}+|\nabla H|_{\beta-1}\right),
$$

for some constant $N$ independent of $s$. Moreover, using Lemma 4.9, we find

$$
\left|r_{1}^{\theta-1} \circ G_{s} \cdot r_{1}^{1-\theta}\right|_{\beta} \leq N\left(1+\left|r_{1}^{-1} H\right|_{0}+|\nabla H|_{\beta-1}\right)^{\theta+\beta} .
$$

The statement then follows.

(4) First, we will consider the case $\theta=0$. By part (1), we have for each $\bar{\mu} \in(0,(\beta+\mu) \wedge 1]$,

$$
\left|\phi \circ \tilde{H}^{-1}-\phi\right|_{0} \leq[\phi]_{\bar{\mu}}\left|H \circ \tilde{H}^{-1}\right|_{0}^{\mu} \leq[\phi]_{\bar{\mu}}|H|_{0}^{\bar{\mu}} .
$$

First, let us consider the case $\beta \leq 1$. For each $x$, let $\mathcal{J}(x)=\phi\left(\tilde{H}^{-1}(x)\right)-\phi(x)$. For all $x$ and $y$, it is clear that

$$
|\mathcal{J}(x)-\mathcal{J}(y)| \leq A(x, y)+B(x, y)+C(x, y)
$$


where

$$
A(x, y):=|\mathcal{J}(x)| \mathbf{1}_{\left[L_{4}, \infty\right)}(|x-y|), \quad B(x, y):=|\mathcal{J}(y)| \mathbf{1}_{\left[L_{4}, \infty\right)}(|x-y|),
$$

and

$$
C(x, y):=|\mathcal{J}(x)-\mathcal{J}(y)| \mathbf{1}_{\left[0, L_{4}\right)}(|x-y|) .
$$

Moreover, owing to part (1), if $\beta+\mu \leq 1$, then for all $x$, and $y$, we have

$$
\begin{gathered}
A(x, y) \leq[\phi]_{\beta+\mu} L_{4}^{\beta+\mu} \mathbf{1}_{\left[L_{4}, \infty\right)}(|x-y|) \leq[\phi]_{\beta+\mu} L_{4}^{\mu}|x-y|^{\{\beta\}^{+}}, \\
B(x, y) \leq[\phi]_{\beta+\mu} L_{4}^{\mu}|x-y|^{\beta},
\end{gathered}
$$

and

$$
\begin{gathered}
C(x, y) \leq[\phi]_{\beta+\mu}\left|\left[\tilde{H}^{-1}\right]_{1}^{\beta+\mu}\right| x-\left.y\right|^{\beta+\mu} \mathbf{1}_{\left[0, L_{4}\right)}(|x-y|)+[\phi]_{\beta+\mu}|x-y|^{\beta+\mu} \mathbf{1}_{\left[0, L_{4}\right)}(|x-y|) \\
\leq N[\phi]_{\beta+\mu} L_{4}^{\mu}|x-y|^{\beta}
\end{gathered}
$$

for some constant $N=N\left(\mu, N_{\kappa}, L_{4}\right)$. Using the identity

$$
\begin{gathered}
\mathcal{J}(x)-\mathcal{J}(y) \\
=-\int_{0}^{1}\left(\nabla \phi\left(x-\theta H\left(\tilde{H}^{-1}(x)\right)\right)-\nabla \phi\left(y-\theta H\left(\tilde{H}^{-1}(y)\right)\right)\right) H\left(\tilde{H}^{-1}(x)\right) d \theta \\
-\int_{0}^{1} \nabla \phi\left(y-\theta H\left(\tilde{H}^{-1}(y)\right)\right)\left(H\left(\tilde{H}^{-1}(y)\right)-H\left(\tilde{H}^{-1}(x)\right)\right),
\end{gathered}
$$

and part (1), if $\beta+\mu>1$, we get that there is a constant $N=N\left(\mu, N_{\kappa}, L_{4}\right)$ such that for all $x$ and $y$,

$$
\begin{gathered}
|\mathcal{J}(x)-\mathcal{J}(y)| \mathbf{1}_{\left[L_{4}, \infty\right)}(|x-y|) \leq N\left([\nabla \phi]_{\beta+\mu-1}|x-y|^{\beta+\mu-1} L_{4}+|\nabla \phi|_{0}|x-y|[H]_{1}\right) \mathbf{1}_{\left[L_{4}, \infty\right)}(|x-y|) \\
\leq N[\nabla \phi]_{\beta+\mu-1} L_{4}^{\mu}|x-y|^{\beta}+N|\nabla \phi|_{0}|\nabla H|_{0}|x-y| .
\end{gathered}
$$

Moreover, since

$$
\begin{gathered}
\mathcal{J}(x)-\mathcal{J}(y) \\
=\int_{0}^{1} \nabla \phi\left(\tilde{H}^{-1}(x+\theta(y-x))\right)\left(\nabla \tilde{H}^{-1}(x+\theta(y-x))-I_{d}\right)(x-y) d \theta \\
+\int_{0}^{1}\left(\nabla \phi\left(\tilde{H}^{-1}(x+\theta(y-x))\right)-\nabla \phi(x+\theta(y-x))\right)(x-y) d \theta,
\end{gathered}
$$

by part (1) and (4.2), if $\beta+\mu>1$, we have that there is a constant $N=N\left(\mu, N_{\kappa}, L_{4}\right)$ such that for all $x$ and $y$,

$$
\begin{gathered}
|\mathcal{J}(x)-\mathcal{J}(y)| \mathbf{1}_{\left[0, L_{4}\right)}(|x-y|) \leq\left(|\nabla \phi|_{0}|\nabla H|_{0}+[\nabla \phi]_{\beta+\mu-1} L_{4}^{\beta+\mu-1}\right)|x-y| \mathbf{1}_{\left[0, L_{4}\right)}(|x-y|) \\
\leq|\nabla \phi|_{0}|\nabla H|_{0}|x-y|+[\nabla \phi]_{\beta+\mu-1} L_{4}^{\mu}|x-y|^{\beta}
\end{gathered}
$$


Combining the above estimates, we get that for all $\beta \leq 1$ and $\mu \in(0,1]$, there is a constant $N=N\left(\mu, N_{\kappa}, L_{4}\right)$ such that

$$
\left[\phi \circ \tilde{H}^{-1}-\phi\right]_{\beta} \leq N \mathbf{1}_{[0,1]}(\beta+\mu)[\phi]_{\beta+\mu} L_{4}^{\mu}+N \mathbf{1}_{(1,2]}(\beta+\mu)\left([\nabla \phi]_{\beta+\mu-1}+|\nabla \phi|_{0}|\nabla H|_{0}\right) .
$$

This proves the desired estimate for $\beta \leq 1$ and $\theta=0$. We now consider the case $\beta>1$. For $\beta>1$, it is straightforward to prove by induction that for all multi-indices $\gamma$ with $1 \leq|\gamma| \leq$ $[\beta]^{-}$and for all $x$,

$$
\partial^{\gamma}\left(\phi\left(\tilde{H}^{-1}\right)\right)(x)=\mathcal{J}_{1}^{\gamma}(x)+\mathcal{J}_{2}^{\gamma}(x)+\mathcal{J}_{3}^{\gamma}(x)+\mathcal{J}_{4}^{\gamma}(x)
$$

where

$$
\begin{gathered}
\mathcal{J}_{1}^{\gamma}(x):=\partial^{\gamma} \phi\left(\tilde{H}^{-1}(x)\right), \\
\mathcal{J}_{2}^{\gamma}(x)=\partial^{\gamma} \phi\left(\tilde{H}^{-1}\right)\left(\partial_{1} \tilde{H}^{-1 ; 1}\right)^{\gamma_{1}} \cdots\left(\partial_{d} \tilde{H}^{-1 ; d}\right)^{\gamma_{d}}-1,
\end{gathered}
$$

$\mathcal{J}_{3}^{\gamma}(x)$ is a finite sum of terms of the form

$$
\partial_{j_{1}} \cdots \partial_{j_{k}} \phi\left(\tilde{H}^{-1}(x)\right) \partial^{\tilde{\gamma}_{1}} \tilde{H}^{-1 ; j_{1}}(x) \cdots \partial^{\tilde{\gamma}_{k}} \tilde{H}^{-1 ; j_{k}}(x)
$$

with $1 \leq k<[\beta]^{-}, j_{1}, \ldots, j_{k} \in\{1, \ldots, d\}$, and $\sum_{j=1}^{k} \tilde{\gamma}_{j}=\gamma$, and $\mathcal{J}_{4}(x)$ is a finite sum of terms of the form

$$
\partial_{j_{1}} \ldots \partial_{j_{[\beta]^{-}}} \phi\left(\tilde{H}^{-1}(x)\right) \partial_{i_{1}} \tilde{H}^{-1 ; j_{1}}(x) \cdots \partial_{i_{[\beta]^{-}}} \tilde{H}^{-1 ; j_{[\beta]^{-}}}(x)
$$

with $i_{1}, j_{1}, \ldots, i_{[\beta]^{-}}, j_{[\beta]^{-}} \in\{1, \ldots, d\}$ and at least one pair $i_{k} \neq j_{k}$. Since for each $x$,

$$
\nabla \tilde{H}^{-1}(x)=I+\nabla F(x)
$$

and (4.2) holds, there is a constant $N=N\left(d_{1}, \beta\right)$ such that

$$
\sum_{1 \leq|\gamma| \leq \beta} \sum_{i=2}^{4}\left|\mathcal{J}_{i}^{\gamma}\right|_{0}+\sum_{|\gamma|=\beta} \sum_{i=2}^{4}\left|\mathcal{J}_{i}^{\gamma}\right|_{\{\beta\}^{+}} \leq N|\nabla \phi|_{\beta-1}|\nabla F|_{\beta-1} \leq N|\nabla \phi|_{\beta-1}|\nabla H|_{\beta-1} .
$$

If $\beta>2$, then for all multi-indices $\gamma$ with $1 \leq|\gamma|<[\beta]^{-}$, we get

$$
\left|\mathcal{J}_{1}^{\gamma}-\partial^{\gamma} \phi\right|_{0}=\left|\partial^{\gamma} \phi \circ \tilde{H}^{-1}-\partial^{\gamma} \phi\right|_{0} \leq\left[\partial^{\gamma} \phi\right]_{1}|H|_{0} .
$$

It is easy to see that there is a constant $N=N\left(L_{4}, N_{\kappa}\right)$ such that for all $\gamma$ with $|\gamma|=[\beta]^{-}$and all $\bar{\mu} \in\left(0,\left(\{\beta\}^{+}+\mu\right) \wedge 1\right]$,

$$
\left|\mathcal{J}_{1}^{\gamma}-\partial^{\gamma} \phi\right|_{0}=\left|\partial^{\gamma} \phi \circ \tilde{H}^{-1}-\partial^{\gamma} \phi\right|_{0} \leq\left[\partial^{\gamma} \phi\right]_{\bar{\mu}}|H|_{0}^{\bar{\mu}} .
$$

Moreover, appealing to the estimate (4.5), we obtain

$$
\begin{gathered}
{\left[\mathcal{J}_{1}^{\gamma}-\partial^{\gamma} \phi\right]_{\{\beta\}^{+}}} \\
\leq N \mathbf{1}_{[0,1]}\left(\{\beta\}^{+}+\mu\right)\left[\partial^{\gamma} \phi\right]_{\{\beta\}^{+}+\mu} L_{4}^{\mu}+N \mathbf{1}_{(1,2]}\left(\{\beta\}^{+}+\mu\right)\left(\left[\nabla \partial^{\gamma} \phi\right]_{\{\beta\}^{+}+\mu-1}+\left|\nabla \partial^{\gamma} \phi\right|_{0}|\nabla H|_{0}\right) .
\end{gathered}
$$


Let us now consider the case $\theta>0$. The following decomposition obviously holds for all $x$ :

$$
r_{1}(x)^{-\theta} \phi\left(\tilde{H}^{-1}(x)\right)-r_{1}(x)^{-\theta} \phi(x)=\hat{\phi}\left(\tilde{H}^{-1}\right)-\hat{\phi}(x)+\left(\frac{r_{1}\left(\tilde{H}^{-1}(x)\right)^{\theta}}{r_{1}(x)^{\theta}}-1\right) \hat{\phi}\left(\tilde{H}^{-1}(x)\right),
$$

where $\hat{\phi}=r_{1}^{-\theta} \phi \in C^{\beta}\left(\mathbf{R}^{d_{1}} ; \mathbf{R}^{d_{1}}\right)$. Thus, to complete the proof we require

$$
\left|\hat{\phi} \circ \tilde{H}^{-1}\right|_{\beta} \leq N|\hat{\phi}|_{\beta} \quad \text { and } \quad\left|\frac{r_{1}^{\theta} \circ \tilde{H}^{-1}}{r_{1}^{\theta}}-1\right|_{\beta} \leq N\left(|H|_{0}+|\nabla H|_{\beta \vee 1-1}\right) .
$$

The latter inequality was proved in part (3) and the first inequality follows from part (2) and Lemma 4.9.

Remark 4.11. Let $H: \mathbf{R}^{d_{1}} \rightarrow \mathbf{R}^{d_{1}}$ be continuously differentiable and assume that for all $x$,

$$
|\nabla H(x)| \leq \eta<1 .
$$

Then for each $x \in \mathbf{R}^{d_{1}}$,

$$
\left|\left(I_{d_{1}}+\nabla H(x)\right)^{-1}\right| \leq\left|I_{d_{1}}+\sum_{k=1}^{\infty}(-1)^{k} \nabla H(x)^{k}\right| \leq \frac{1}{1-\eta} .
$$

\subsection{Stochastic Fubini thoerem}

Let $m=\left(m^{\varrho}\right)_{t \leq T}, \varrho \geq 1$, be a sequence of $\mathbf{F}$-adapted locally square integrable continuous martingales issuing from zero such that P-a.s. for all $t \in[0, T],\left\langle m^{\varrho_{1}}, m^{\varrho_{2}}\right\rangle_{t}=0$ for $\varrho_{1} \neq \varrho_{2}$ and $\left\langle m^{\varrho}\right\rangle_{t}=N_{t}$ for $\varrho \geq 1$, where $N_{t}$ is a $\mathcal{P}_{T}$-measurable continuous increasing processes issuing from zero. Let $\eta(d t, d z)$ be a $\mathbf{F}$-adapted integer-valued random measure on $([0, T] \times$ $E, \mathcal{B}([0, T]) \otimes \mathcal{E})$, where $(U, \mathcal{U})$ is a Blackwell space. We assume that $\eta(d t, d z)$ is optional, $\tilde{\mathcal{P}}_{T}$-sigma-finite, and quasi-left continuous. Thus, there exists a unique (up to a $\mathbf{P}$-null set) dual predictable projection (or compensator) $\eta^{p}(d t, d z)$ of $\eta(d t, d z)$ such that $\mu(\omega,\{t\} \times U)=0$ for all $\omega$ and $t$. We refer the reader to Ch. II, Sec. 1, in [JS03] for any unexplained concepts relating to random measures.

Let $(X, \Sigma, \mu)$ be a sigma-finite measure space; that is, there is an increasing sequence of $\Sigma$-measurable sets $X_{n}, n \in \mathbf{N}$, such that $X=\cup_{n=1}^{\infty} X_{n}$ and $\mu\left(X_{n}\right)<\infty$ for each $n$. Let $f$ : $\Omega \times[0, T] \times X \rightarrow \mathbf{R}^{d_{2}}$ be $\mathcal{R}_{T} \otimes \Sigma$-measurable, $g: \Omega \times[0, T] \times X \rightarrow \ell_{2}\left(\mathbf{R}^{d_{2}}\right)$ be $\mathcal{R}_{T} \otimes \Sigma / \mathcal{B}\left(\ell_{2}\left(\mathbf{R}^{d_{2}}\right)\right)$ measurable, and $h: \Omega \times[0, T] \times X \times U \rightarrow \mathbf{R}^{d_{2}}$ be $\mathcal{P}_{T} \otimes \Sigma \otimes \mathcal{U}$-measurable. Moreover, assume that for each $t \in[0, T]$ and $x \in X, \mathbf{P}$-a.s.

$$
\int_{] 0, T]}\left|g_{t}(x)\right|^{2} d N_{t}+\int_{] 0, T]} \int_{U}\left|h_{t}(x, z)\right|^{2} \eta^{p}(d t, d z)<\infty .
$$

Let $F=F_{t}(x): \Omega \times[0, T] \times X \rightarrow \mathbf{R}^{d_{2}}$ be $O_{T} \otimes \mathcal{B}(X)$-measurable and assume that for $d \mathbf{P} \otimes \mu$-almost all $(t, x) \in[0, T] \times X$,

$$
F_{t}(x)=\int_{] 0, t]} g_{s}^{\varrho}(x) d m_{s}^{\varrho}+\int_{] 0, t]} \int_{U} h_{s}(x, z) \tilde{\eta}(d t, d z)
$$


where $\tilde{\eta}(d t, d z)=\eta(d t, d z)-\eta^{p}(d t, d z)$.

The following version of the stochastic Fubini theorem is a straightforward extension of Lemma 2.6 [Kry11] and Corollary 1 in [Mik83]. See also Proposition 3.1 in [Zho13], Theorem 2.2 in [Ver12], and Theorem 1.4.8 in [Roz90]. Indeed, to prove it for a bounded measure, we can use a monotone class argument as in Theorem 64 in [Pro05]. To handle the general setting with possibly infinite $\mu$, we use assumptions (ii) and (iii) below and take limits on the sets $X_{n}$ using the Lenglart domination lemma Lenglart domination lemma (Theorem 1.4.5 on page 66 in [LS89]) and the following well known inequalities:

$$
\begin{aligned}
\mathbf{E} \sup _{t \leq T}\left|\int_{] 0, t]} g_{s}^{\varrho} d w_{s}^{\varrho}\right| & \leq N \mathbf{E}\left(\int_{] 0, T]}\left|g_{t}(x)\right|^{2} d w_{t}^{\varrho}\right)^{1 / 2} \\
\mathbf{E} \sup _{t \leq T}\left|\int_{] 0, t]} \int_{U} h_{t}(x, z) \tilde{\eta}(d t, d z)\right| & \leq N \mathbf{E}\left(\int_{] 0, T]} \int_{U}\left|h_{t}(x, z)\right|^{2} \eta^{p}(d t, d z)\right)^{1 / 2},
\end{aligned}
$$

where $\tau \leq T$ is an arbitrary stopping time and $N=N(T)$ is a constant independent of $g$ and $h$.

Proposition 4.12 (c.f. Corollary 1 in [Mik83] and Lemma 2.6 in [Kry11]). Assume that

(1) P-a.s. for each $n \geq 1$,

$$
\int_{X_{n}}\left(\int_{] 0, T]}\left|g_{t}(x)\right|^{2} d N_{t}\right)^{1 / 2} \mu(d x)+\int_{X_{n}}\left(\int_{] 0, T]} \int_{U_{1}}\left|h_{t}(x, z)\right|^{2} \eta^{p}(d t, d z)\right)^{1 / 2} \mu(d x)<\infty
$$

(2) P-a.s.

$$
\int_{] 0, T]}\left(\int_{X}\left|g_{t}(x)\right| \mu(d x)\right)^{2} d t+\int_{] 0, T]} \int_{U}\left(\int_{X}\left|h_{t}(x, z)\right| \mu(d x)\right)^{2} \eta^{p}(d t, d z) ;
$$

(3) P-a.s. for al $t \in[0, T]$,

$$
\int_{X}\left|F_{t}(x)\right| \mu(d x)<\infty
$$

Then $\mathbf{P}$-a.s. for all $t \in[0, T]$,

$$
\int_{X} F_{t}(x) \mu(d x)=\int_{] 0, t]} \int_{X} g_{s}^{\varrho}(x) \mu(d x) d m_{s}^{\varrho}+\int_{] 0, t]} \int_{U} \int_{X} h_{s}(x, z) \mu(d x) \tilde{\eta}(d r, d z)
$$

We obtain the following corollary by applying Minkowski's integral inequaility.

Corollary 4.13. Assume that P-a.s.

$$
\int_{X}\left(\int_{] 0, T]}\left|g_{t}(x)\right|^{2} d N_{t}\right)^{1 / 2} \mu(d x)+\int_{X}\left(\int_{10, T]} \int_{U_{1}}\left|h_{t}(x, z)\right|^{2} \eta^{p}(d t, d z)\right)^{1 / 2} \mu(d x)<\infty .
$$

Then P-a.s. for all $t \in[0, T]$,

$$
\int_{X} F_{t}(x) \mu(d x)=\int_{] 0, t]} \int_{X} g_{s}^{\varrho}(x) \mu(d x) d m_{s}^{\varrho}+\int_{] 0, t]} \int_{U} \int_{X} h_{s}(x, z) \mu(d x) \tilde{\eta}(d r, d z) .
$$


Remark 4.14. If $\mu$ is a finite-measure and $\mathbf{P}$-a.s.

$$
\int_{X} \int_{] 0, T]}\left|g_{t}(x)\right|^{2} d N_{t} \mu(d x)+\int_{X} \int_{] 0, T]} \int_{U_{1}}\left|h_{t}(x, z)\right|^{2} \eta^{p}(d t, d z) \mu(d x)<\infty,
$$

then (4.6) holds by Hölder's inequality.

\subsection{Itô-Wentzell formula}

Definition 4.15. We say that an $\mathbf{R}^{d_{1}}$-valued $\mathbf{F}$-adapted quasi-left continuous semimartingale $L_{t}=\left(L_{t}^{k}\right)_{1 \leq k \leq d_{1}}, t \geq 0$, is of $\alpha$-order for some $\alpha \in(0,2]$ if $\mathbf{P}$-a.s. for all $t \geq 0$,

$$
\sum_{s \leq t}\left|\Delta L_{s}\right|^{\alpha}<\infty
$$

and

$$
\begin{aligned}
& L_{t}=L_{0}+\int_{] 0, t]} \int_{\mathbf{R}_{0}^{d_{1}}} z p^{L}(d s, d z), \text { if } \alpha \in(0,1), \\
& L_{t}=L_{0}+A_{t}+\int_{[0, t]} \int_{|z| \leq 1} z q^{L}(d s, d z)+\int_{[0, t]} \int_{|z|>1} z p^{L}(d s, d z), \text { if } \alpha \in[1,2), \\
& L_{t}=L_{0}+A_{t}+L_{t}^{c}+\int_{[0, t]} \int_{|z| \leq 1} z q^{L}(d s, d z)+\int_{[0, t]} \int_{|z|>1} z p^{L}(d s, d z), \text { if } \alpha=2,
\end{aligned}
$$

where $p^{L}(d t, d z)$ is the jump measure of $L$ with dual predictable projection $\pi^{L}(d t, d z), q^{L}$ $(d t, d z)=p^{L}(d t, d z)-\pi^{L}(d t, d z)$ is a martingale measure, $A_{t}=\left(A_{t}^{i}\right)_{1 \leq i \leq d_{1}}$ is a continuous process of finite variation with $A_{0}=0$, and $L_{t}^{c}=\left(L_{t}^{c ; i}\right)_{1 \leq i \leq d_{1}}$ is a continuous local martingale issuing from zero.

Set $\left(w^{\varrho}\right)_{\varrho \geq 1}=\left(w^{1 ; \varrho}\right)_{\varrho \geq 1},(Z, \mathcal{Z}, \pi)=\left(\mathcal{Z}^{1}, \mathcal{Z}^{1}, \pi^{1}\right), p(d t, d z)=p^{1}(d t, d z)$, and $q(d t, d z)=$ $q^{1}(d t, d z)$. Also, set $D=D^{1}, E=E^{1}$, and assume $Z=D \cup E$.

Let $f: \Omega \times[0, T] \times \mathbf{R}^{d_{1}} \rightarrow \mathbf{R}^{d_{2}}$ be $\mathcal{R}_{T} \otimes \mathcal{B}\left(\mathbf{R}^{d_{1}}\right)$-measurable, $g: \Omega \times[0, T] \times \mathbf{R}^{d_{1}} \rightarrow \ell_{2}\left(\mathbf{R}^{d_{2}}\right)$ be $\mathcal{R}_{T} \otimes \mathcal{B}\left(\mathbf{R}^{d_{1}}\right) / \mathcal{B}\left(\ell_{2}\left(\mathbf{R}^{d_{2}}\right)\right)$-measurable, and $h: \Omega \times[0, T] \times \mathbf{R}^{d_{1}} \times Z \rightarrow \mathbf{R}^{d_{2}}$ be $\mathcal{P}_{T} \otimes \mathcal{B}\left(\mathbf{R}^{d_{1}}\right) \otimes \mathcal{Z}$ measurable. Moreover, assume that, $\mathbf{P}$-a.s. for all $x \in \mathbf{R}^{d_{1}}$,

$$
\int_{] 0, T]}\left|f_{t}(x)\right| d t+\int_{] 0, T]}\left|g_{t}(x)\right|^{2} d t+\int_{] 0, T]} \int_{D}\left|h_{t}(x, z)\right|^{2} \pi(d z) d t+\int_{] 0, T]} \int_{E}\left|h_{t}(x, z)\right| \pi(d z) d t<\infty .
$$

Let $F=F_{t}(x): \Omega \times[0, T] \times \mathbf{R}^{d_{1}} \rightarrow \mathbf{R}^{d_{2}}$ be $O_{T} \otimes \mathcal{B}\left(\mathbf{R}^{d_{1}}\right)$-measurable and assume that for each $x, \mathbf{P}$-a.s. for all $t$,

$F_{t}(x)=F_{0}(x)+\int_{] 0, t]} f_{s}(x) d s+\int_{] 0, t]} g_{s}^{\varrho}(x) d w_{s}^{\varrho}+\int_{] 0, t]} \int_{Z} h_{s}(x, z)\left[\mathbf{1}_{D}(z) q(d s, d z)+\mathbf{1}_{E}(z) p(d s, d z)\right]$.

For each $n \in\{1,2\}$, let $C_{l o c}^{n}\left(\mathbf{R}^{d_{1}} ; \mathbf{R}^{d_{2}}\right)$ be space of $n$-times continuously differentiable functions $f: \mathbf{R}^{d_{1}} \rightarrow \mathbf{R}^{d_{2}}$. We now state our version of the Itô-Wentzell formula. For each $\omega, t$ and $x$, we denote $\Delta F(x)=F_{t}(x)-F_{t-}(x)$. 
Proposition 4.16 (cf. Proposition 1 in [Mik83] ). Let $\left(L_{t}\right)_{t \geq 0}$ be an $\mathbf{R}^{d_{1}}$-valued quasi-left continuous semimartingale of order $\alpha \in(0,2]$. Assume that:

(1) (a) P-a.s. $F \in D\left([0, T] ; C_{l o c}^{\alpha}\left(\mathbf{R}^{d} ; \mathbf{R}^{m}\right)\right.$ if $\alpha$ is fractional and $F \in D\left([0, T] ; C_{l o c}^{\alpha}\left(\mathbf{R}^{d} ; \mathbf{R}^{m}\right)\right.$ if $\alpha=1,2$;

(b) for d $\mathbf{P}$ dt-almost-all $(\omega, t) \in \Omega \times[0, T], f_{t}(x)$ and $g_{t}(x)=\left(g_{t}^{i \varrho}(x)\right)_{\varrho \geq 1} \in \ell_{2}\left(\mathbf{R}^{d_{2}}\right)$ are continuous in $x$ and

$$
d \mathbf{P} d t-\lim _{y \rightarrow x}\left[\int_{D}\left|h_{t}(y, z)-h_{t}(x, z)\right|^{2} \pi(d z)+\int_{E}\left|h_{t}(y, z)-h_{t}(x, z)\right| \pi(d z)\right]=0 ;
$$

(c) for each $\rho \geq 1$ and $i \in\left\{1, \ldots, d_{1}\right\}$ and for $d \mathbf{P} d\left|\left\langle L^{c, i}, w^{\varrho}\right\rangle\right|_{t}$-almost-all $(\omega, t) \in \Omega \times$ $[0, T], g_{t}^{i \rho} \in C_{l o c}^{1}\left(\mathbf{R}^{d} ; \mathbf{R}\right)$, if $\alpha=2$, ;

(2) for each compact subset $K$ of $\mathbf{R}^{d_{1}}, \mathbf{P}$-a.s.

$$
\begin{gathered}
\int_{10, T]} \sup _{x \in K}\left(\left|f_{t}(x)\right|+\left|g_{t}(x)\right|^{2}+\int_{D}\left|h_{t}(x, z)\right|^{2} \pi(d z)+\int_{E}\left|h_{t}(x, z)\right| \pi(d z)\right) d t<\infty, \\
\sum_{\varrho \geq 1} \int_{10, T]} \sup _{x \in K}\left|\nabla g_{t}^{i \varrho}(x)\right| d\left|\left\langle L^{c ; i}, w^{\varrho}\right\rangle\right|_{t}+\sum_{t \leq T}\left|\Delta F_{t}\right|_{\alpha \wedge 1 ; K}\left|\Delta L_{t}\right|^{\alpha \wedge 1}<\infty .
\end{gathered}
$$

Then $\mathbf{P}$-a.s for all $t \in[0, T]$,

$$
\begin{aligned}
F_{t}\left(L_{t}\right)= & F_{0}\left(L_{0}\right)+\int_{10, t]} f_{s}\left(L_{s}\right) d s+\int_{10, t]} g_{s}^{\varrho}\left(L_{s}\right) d w_{s}^{\varrho} \\
& +\int_{[0, t]} \int_{Z} h_{s}\left(L_{s-}, z\right)\left[\mathbf{1}_{D}(z) q(d r, d z)+\mathbf{1}_{E}(z) p(d r, d z)\right] \\
& +\int_{0, t]} \partial_{i} F_{s-}\left(L_{s-}\right)\left[\mathbf{1}_{[1,2]}(\alpha) d A_{s}^{i}+\mathbf{1}_{\{2\}}(\alpha) d L_{s}^{c i,}\right]+\mathbf{1}_{\{2\}}(\alpha) \frac{1}{2} \int_{[0, t]} \partial_{i j} F_{s}\left(L_{s}\right) d\left\langle L^{c ; i}, L^{c ; j}\right\rangle_{s} \\
& +\sum_{s \leq t}\left(F_{s-}\left(L_{s}\right)-F_{s-}\left(L_{s-}\right)-\mathbf{1}_{[1,2]}(\alpha) \nabla F_{s-}\left(L_{s-}\right) \Delta L_{s}\right) \\
& +\mathbf{1}_{\{2\}}(\alpha) \int_{10, t]} \partial_{i} g_{s}^{\varrho}\left(L_{s}\right) d\left\langle w^{o}, L^{c ; i}\right\rangle_{s}+\sum_{s \leq t}\left(\Delta F_{s}\left(L_{s}\right)-\Delta F_{s}\left(L_{s-}\right)\right) .
\end{aligned}
$$

Proof. Since both sides have identical jumps and we can always interlace a finite set of jumps, we may assume that $\left|\Delta L_{t}\right| \leq 1$ for all $t \in[0, T]$; that is, it is enough to prove the statement for $\tilde{L}_{t}=L_{t}-\sum_{s \leq t} \mathbf{1}_{[1, \infty)}\left(\left|\Delta L_{s}\right|\right) \Delta L_{s}, t \in[0, T]$. It suffices to assume that for some $K$ and all $\omega,\left|L_{0}\right| \leq K$. For each $R>K$, let

$$
\tau_{R}=\inf \left(t \in[0, T]:|A|_{t}+\left|\left\langle L^{c}\right\rangle\right|_{t}+\sum_{s \leq t}\left|\Delta L_{s}\right|^{\alpha}+\left|L_{t}\right|>R\right) \wedge T
$$

and note that $\mathbf{P}$-a.s. $\tau_{R} \uparrow T$ as $R$ tends to infinity. If instead of $L, f, g, h$, and $F$, we take $L_{\cdot \wedge \tau_{R}}, f \mathbf{1}_{\left(0, \tau_{R}\right]}, g^{\varrho} \mathbf{1}_{\left(0, \tau_{R}\right]}, h \mathbf{1}_{\left(0, \tau_{R}\right]}, F \mathbf{1}_{\left(0, \tau_{R}\right]}$, then the assumptions of the proposition hold for this 
new set of processes. Moreover, if we can prove (4.7) for this new set of processes, then by taking the limit as $R$ tends to infinity, we obtain (4.7). Therefore, we may assume that for some $R>0, \mathbf{P}$-a.s. for all $t \in[0, T]$,

$$
|A|_{t}+\left|\left\langle L^{c}\right\rangle\right|_{t}+\sum_{s \leq t}\left|\Delta L_{s}\right|^{\alpha}+\left|L_{t}\right| \leq R
$$

Let $\phi \in C_{c}^{\infty}\left(\mathbf{R}^{d_{1}}, \mathbf{R}\right)$ have support in the unit ball in $\mathbf{R}^{d_{1}}$ and satisfy $\int_{\mathbf{R}^{d_{1}}} \phi(x) d x=1, \phi(x)=$ $\phi(-x)$, and $\phi(x) \geq 0$, for all $x \in \mathbf{R}^{d_{1}}$. For each $\varepsilon \in(0,1)$, let $\phi_{\varepsilon}(x)=\varepsilon^{-d} \phi(x / \varepsilon), x \in \mathbf{R}^{d_{1}}$. By Itô's formula, for each $x \in \mathbf{R}^{d_{1}}, \mathbf{P}$-a.s. for all $t \in[0, T]$,

$$
\begin{aligned}
F_{t}(x) \phi_{\varepsilon}\left(x-L_{t}\right)= & F_{0}(x) \phi_{\varepsilon}\left(x-L_{0}\right)-\int_{] 0, t]} F_{s-}(x) \partial_{i} \phi_{\varepsilon}\left(x-L_{s-}\right) d L_{s}^{i} \\
& +\mathbf{1}_{\{2\}}(\alpha) \frac{1}{2} \int_{] 0, t]} F_{s}(x) \partial_{i j} \phi_{\varepsilon}\left(x-L_{s}\right) d\left\langle L^{c ; i}, L^{c ; j}\right\rangle_{s}+\int_{] 0, t]} \phi_{\varepsilon}\left(x-L_{s}\right) f_{s}(x) d s \\
& +\mathbf{1}_{\{2\}}(\alpha) \int_{] 0, t]} g_{s}^{\varrho}(x) \partial_{i} \phi_{\varepsilon}\left(x-L_{s}\right) d\left\langle w^{\varrho}, L^{c ; i}\right\rangle_{s}+\int_{] 0, t]} \phi_{\varepsilon}\left(x-L_{s}\right) g_{s}^{\varrho}(x) d w_{s}^{\varrho} \\
& +\int_{] 0, t]} \int_{Z} \phi_{\varepsilon}\left(x-L_{s-}\right) h_{s}(x, z)\left[\mathbf{1}_{D}(z) q(d r, d z)+\mathbf{1}_{E}(z) p(d r, d z)\right] \\
& +\sum_{s \leq t} \Delta F_{s}(x)\left(\phi_{\varepsilon}\left(x-L_{s}\right)-\phi_{\varepsilon}\left(x-L_{s-}\right)\right) \\
& +\sum_{s \leq t} F_{s-}(x)\left(\phi_{\varepsilon}\left(x-L_{s}\right)-\phi_{\varepsilon}\left(x-L_{s-}\right)+\partial_{i} \phi_{\varepsilon}\left(x-L_{s-}\right) \Delta L_{s}\right) .
\end{aligned}
$$

Appealing to assumption (2) and (4.8) (i.e. for the integrals against $F$ ), we integrate both sides of the above in $x$, apply Corollary 4.13 (see, also, Remark 4.14) and the deterministic Fubini theorem, and then integrate by parts to get that $\mathbf{P}$-a.s. for all $t \in[0, T]$,

$$
\begin{aligned}
F_{t}^{(\varepsilon)}\left(L_{t}\right)= & F_{0}^{(\varepsilon)}\left(L_{0}\right)+\int_{] 0, t]} \nabla F_{s-}^{(\varepsilon)}\left(L_{s-}\right)\left[\mathbf{1}_{[1,2]}(\alpha) d A_{s}^{i}+\mathbf{1}_{\{2\}}(\alpha) d L_{s}^{c ; i}\right]+\int_{] 0, t]} f_{s}^{(\varepsilon)}\left(L_{s}\right) d r \\
& +\int_{] 0, t]} g_{s}^{(\varepsilon)}\left(L_{s}\right) d w_{s}^{\varrho}+\int_{] 0, t]} \int_{Z} h_{s}^{(\varepsilon)}\left(L_{s-}, z\right)\left[\mathbf{1}_{D}(z) q(d r, d z)+\mathbf{1}_{E}(z) p(d r, d z)\right] \\
& +\mathbf{1}_{\{2\}}(\alpha) \frac{1}{2} \int_{] 0, t]} \partial_{i j} F_{s}^{(\varepsilon)}\left(L_{s}\right) d\left\langle L^{c ; i}, L^{c ; j}\right\rangle_{s}+\mathbf{1}_{\{2\}}(\alpha) \int_{] 0, t]} \partial_{i} g_{s}^{(\varepsilon) ; \varrho}\left(L_{s}\right) d\left\langle w^{\varrho}, L^{c ; i}\right\rangle_{s} \\
& +\sum_{s \leq t}\left(\Delta F_{s}^{(\varepsilon)}\left(L_{s}\right)-\Delta F_{s}^{(\varepsilon)}\left(L_{s-}\right)\right) \\
& +\sum_{s \leq t}\left(F_{s-}^{(\varepsilon)}\left(L_{s}\right)-F_{s-}^{(\varepsilon)}\left(L_{s-}\right)-\mathbf{1}_{[1,2]}(\alpha) \nabla F_{s-}^{(\varepsilon)}\left(L_{s-}\right) \Delta L_{s}\right)
\end{aligned}
$$

where for each $\omega, t, x$, and $z$,

$$
F_{t}^{(\varepsilon)}(x):=\phi_{\varepsilon} * F_{t}(x), \quad f_{t}^{(\varepsilon)}=\phi_{\varepsilon} * f_{t}(x), \quad g_{t}^{(\varepsilon) ; \underline{Q}}(x)=\phi_{\varepsilon} * g_{t}^{\varrho}(x), \quad h_{t}^{(\varepsilon)}(x, z)=\phi_{\varepsilon} * h_{t}(x, z),
$$


and $*$ denotes the convolution operator on $\mathbf{R}^{d_{1}}$. Let $B_{R+1}=\left\{x \in \mathbf{R}^{d_{1}}:|x| \leq R+1\right\}$. Owing to assumption (1)(a) and standard properties of mollifiers, for each multi-index $\gamma$ with $|\gamma| \leq \alpha$, $\mathbf{P}$-a.s. for all $t$,

$$
\left|\partial^{\gamma} F_{t}^{(\varepsilon)}\left(L_{t}\right)\right| \leq \sup _{t \leq T} \sup _{x \in B_{R+1}}\left|\partial^{\gamma} F_{t}(x)\right|<\infty
$$

and for each $x$,

$$
d \mathbf{P} d t-\lim _{\varepsilon \downarrow 0}\left|\partial^{\gamma} F_{t}^{(\varepsilon)}(x)-\partial^{\gamma} F_{t}^{(\varepsilon)}(x)\right|=0 .
$$

Similarly, by assumption $1(\mathrm{~b}), d \mathbf{P} d t$-almost-all $(\omega, t) \in \Omega \times[0, T]$,

$$
\begin{gathered}
\left|f_{t}^{(\varepsilon)}\left(L_{t}\right)\right| \leq \sup _{x \in B_{R+1}}\left|f_{t}(x)\right|<\infty, \quad\left|g_{t}^{(\varepsilon)}\left(L_{t}\right)\right| \leq \sup _{x \in B_{R+1}}\left|g_{t}(x)\right|<\infty, \\
\int_{D}\left|h_{t}^{\varepsilon}\left(L_{t}, z\right)\right|^{2} \pi(d z) \leq \sup _{x \in B_{R+1}} \int_{D}\left|h_{t}(x, z)\right|^{2} \pi(d z) \\
\int_{E}\left|h_{t}^{\varepsilon}\left(L_{t}, z\right)\right| \pi(d z) \leq \sup _{x \in B_{R+1}} \int_{E}\left|h_{t}(x, z)\right| \pi(d z)
\end{gathered}
$$

and for each $x$,

$$
d \mathbf{P} d t-\lim _{\varepsilon \downarrow 0}\left|f_{t}^{(\varepsilon)}(x)-f_{t}(x)\right|=0, \quad d \mathbf{P} d t-\lim _{\varepsilon \rightarrow 0}\left|g_{t}^{(\varepsilon)}(x)-g_{t}(x)\right|=0
$$

and

$$
d \mathbf{P} d t-\lim _{\varepsilon \downarrow 0} \int_{Z}\left[\mathbf{1}_{D}(z)\left|h_{t}^{(\varepsilon)}(x, z)-h_{t}(x, z)\right|^{2}+\mathbf{1}_{E}(z)\left|h_{t}^{(\varepsilon)}(x, z)-h_{t}(x, z)\right|\right] \pi(d z)=0,
$$

where in the last-line we have also used Minkowski's integral inequality and a standard mollifying convergence argument. Using assumption $1(\mathrm{~d})$, for each $\rho \geq 1$ and $i \in\left\{1, \ldots, d_{1}\right\}$ and for $d \mathbf{P} d\left|\left\langle L^{c ; i}, w^{\varrho}\right\rangle\right|_{t}$-almost-all $(\omega, t) \in \Omega \times[0, T]$

$$
\left|\nabla g_{t}^{(\varepsilon) ; i \varrho}\left(L_{t}\right)\right| \leq \sup _{x \in B_{R+1}}\left|\nabla g_{t}^{i \varrho}(x)\right|
$$

and for each $x$,

$$
d \mathbf{P} d\left|\left\langle L^{c ; i}, w^{\varrho}\right\rangle\right|_{t}-\lim _{\varepsilon \rightarrow 0}\left|\nabla g_{t}^{(\varepsilon) ; i \varrho}(x)-\nabla g_{t}^{i \varrho}(x)\right|=0, \text { if } \alpha=2 .
$$

Owing to assumption 1(a) and (4.8), P-a.s.

$$
\begin{gathered}
\sum_{s \leq t}\left|F_{s-}^{(\varepsilon)}\left(L_{s}\right)-F_{s-}^{(\varepsilon)}\left(L_{s-}\right)-\mathbf{1}_{[1,2]}(\alpha) \nabla F_{s-}^{(\varepsilon)}\left(L_{s-}\right) \Delta L_{s}\right| \\
\quad \leq \sup _{t \leq T}\left|F_{t}\right|_{\alpha ; B_{R+1}} \sum_{s \leq t}\left|\Delta L_{s}\right|^{\alpha} \leq R \sup _{t \leq T}\left|F_{t}\right|_{\alpha ; B_{R+1}} .
\end{gathered}
$$

Since P-a.s. $F \in D\left([0, T] ; C^{\alpha}\left(\mathbf{R}^{d} ; \mathbf{R}^{m}\right)\right.$, it follows that for each $x, \mathbf{P}$-a.s. for all $t$,

$$
\lim _{\varepsilon \downarrow 0}\left|\Delta F_{t}^{\varepsilon}(x)-\Delta F_{t}(x)\right|=0 .
$$


By assumption (2), P-a.s for all $t$, we have

$$
\sum_{s \leq t}\left(\Delta F_{s}^{(\varepsilon)}\left(L_{s-}+\Delta L_{s}\right)-\Delta F_{s}^{(\varepsilon)}\left(L_{s-}\right)\right) \leq \sum_{s \leq t}\left|\Delta F_{t}\right|_{\alpha \wedge 1 ; B_{R+1}}\left|\Delta L_{s}\right|^{\alpha \wedge 1} .
$$

Combining the above and using assumptions (1)(a) and (2) and the bounds given in (4.8) and the deterministic and stochastic dominated convergence theorem, we obtain convergence of all the terms in (4.9), which complete the proof.

\section{References}

[BvNVW08] Z. Brzeźniak, J. M. A. M. van Neerven, M. C. Veraar, and L. Weis. Itô's formula in UMD Banach spaces and regularity of solutions of the Zakai equation. J. Differential Equations, 245(1):30-58, 2008.

[CK10] Zhen-Qing Chen and Kyeong-Hun Kim. An 1 p-theory of non-divergence form spdes driven by lévy processes. arXiv preprint arXiv:1007.3295, 2010.

[DHI13] Arnaud Debussche, Michael Högele, and Peter Imkeller. The dynamics of nonlinear reaction-diffusion equations with small Lévy noise, volume 2085 of Lecture Notes in Mathematics. Springer, Cham, 2013.

[DPMT07] Giuseppe Da Prato, Jose-Luis Menaldi, and Luciano Tubaro. Some results of backward Itô formula. Stoch. Anal. Appl., 25(3):679-703, 2007.

[DPZ92] Giuseppe Da Prato and Jerzy Zabczyk. Stochastic equations in infinite dimensions, volume 44 of Encyclopedia of Mathematics and its Applications. Cambridge University Press, Cambridge, 1992.

[GM11] B. Grigelionis and R. Mikulevicius. Nonlinear filtering equations for stochastic processes with jumps. In The Oxford handbook of nonlinear filtering, pages 95-128. Oxford Univ. Press, Oxford, 2011.

[Gri76] B. Grigelionis. Reduced stochastic equations of nonlinear filtering of random processes. Litovsk. Mat. Sb., 16(3):51-63, 232, 1976.

[Gyö82] I. Gyöngy. On stochastic equations with respect to semimartingales. III. Stochastics, 7(4):231-254, 1982.

[Hau05] Erika Hausenblas. Existence, uniqueness and regularity of parabolic SPDEs driven by Poisson random measure. Electron. J. Probab., 10:1496-1546, 2005.

[HØUZ10] Helge Holden, Bernt Øksendal, Jan Ubøe, and Tusheng Zhang. Stochastic partial differential equations. Universitext. Springer, New York, second edition, 2010. A modeling, white noise functional approach. 
[Jac79] Jean Jacod. Calcul stochastique et problèmes de martingales, volume 714 of Lecture Notes in Mathematics. Springer, Berlin, 1979.

[JS03] Jean Jacod and Albert N. Shiryaev. Limit theorems for stochastic processes, volume 288 of Grundlehren der Mathematischen Wissenschaften [Fundamental Principles of Mathematical Sciences]. Springer-Verlag, Berlin, second edition, 2003.

[KR77] N. V. Krylov and B. L. Rozovskil. The Cauchy problem for linear stochastic partial differential equations. Izv. Akad. Nauk SSSR Ser. Mat., 41(6):13291347, 1448, 1977.

[KR81] N. V. Krylov and B. L. Rozovskiǔ. On the first integrals and Liouville equations for diffusion processes. In Stochastic differential systems (Visegrád, 1980), volume 36 of Lecture Notes in Control and Information Sci., pages 117-125. Springer, Berlin-New York, 1981.

[Kry99] N. V. Krylov. An analytic approach to SPDEs. In Stochastic partial differential equations: six perspectives, volume 64 of Math. Surveys Monogr., pages 185242. Amer. Math. Soc., Providence, RI, 1999.

[Kry11] N. V. Krylov. On the Itô-Wentzell formula for distribution-valued processes and related topics. Probab. Theory Related Fields, 150(1-2):295-319, 2011.

[Kun81] Hiroshi Kunita. On the decomposition of solutions of stochastic differential equations. In Stochastic integrals (Proc. Sympos., Univ. Durham, Durham, 1980), volume 851 of Lecture Notes in Math., pages 213-255. Springer, Berlin-New York, 1981.

[Kun86] H. Kunita. Lectures on stochastic flows and applications, volume 78 of Tata Institute of Fundamental Research Lectures on Mathematics and Physics. Published for the Tata Institute of Fundamental Research, Bombay; by Springer-Verlag, Berlin, 1986.

[Kun04] Hiroshi Kunita. Stochastic differential equations based on Lévy processes and stochastic flows of diffeomorphisms. In Real and stochastic analysis, Trends Math., pages 305-373. Birkhäuser Boston, Boston, MA, 2004.

[LM14a] James-Michael Leahy and Remigijus Mikulevicius. On degenerate linear stochastic evolutions equations driven by jump processes. arXiv preprint arXiv:1406.4541, 2014.

[LM14b] James-Michael Leahy and Remigijus Mikulevicius. On some properties of space inverses of stochastic flows. arXiv preprint arXiv:1411.6277, 2014. 
[LS89] R. Sh. Liptser and A. N. Shiryayev. Theory of martingales, volume 49 of Mathematics and its Applications (Soviet Series). Kluwer Academic Publishers Group, Dordrecht, 1989. Translated from the Russian by K. Dzjaparidze [Kacha Dzhaparidze].

[MB07] Thilo Meyer-Brandis. Stochastic Feynman-Kac equations associated to LévyItô diffusions. Stoch. Anal. Appl., 25(5):913-932, 2007.

[Mey76] P. A. Meyer. La théorie de la prédiction de F. Knight. In Séminaire de Probabilités, X (Première partie, Univ. Strasbourg, Strasbourg, année universitaire 1974/1975), pages 86-103. Lecture Notes in Math., Vol. 511. Springer, Berlin, 1976.

[Mik83] R. Mikulevičius. Properties of solutions of stochastic differential equations. Litovsk. Mat. Sb., 23(4):18-31, 1983.

[Mik00] R. Mikulevicius. On the Cauchy problem for parabolic SPDEs in Hölder classes. Ann. Probab., 28(1):74-103, 2000.

[MP09] R. Mikulevicius and H. Pragarauskas. On Hölder solutions of the integrodifferential Zakai equation. Stochastic Process. Appl., 119(10):3319-3355, 2009.

[MP76] Michel Métivier and Giovanni Pistone. Une formule d'isométrie pour l'intégrale stochastique hilbertienne et équations d'évolution linéaires stochastiques. Z. Wahrscheinlichkeitstheorie und Verw. Gebiete, 33(1):1-18, 1975/76.

[Par72] Étienne Pardoux. Sur des équations aux dérivées partielles stochastiques monotones. C. R. Acad. Sci. Paris Sér. A-B, 275:A101-A103, 1972.

[Par75] Étienne Pardoux. Équations aux dérivées partielles stochastiques de type monotone. In Séminaire sur les Équations aux Dérivées Partielles (19741975), III, Exp. No. 2, page 10. Collège de France, Paris, 1975.

[Pri12] Enrico Priola. Pathwise uniqueness for singular SDEs driven by stable processes. Osaka J. Math., 49(2):421-447, 2012.

[Pri14] Enrico Priola. Stochastic flow for SDEs with jumps and irregular drift term. arXiv preprint arXiv:1405.2575, 2014.

[Pro05] Philip E. Protter. Stochastic integration and differential equations, volume 21 of Stochastic Modelling and Applied Probability. Springer-Verlag, Berlin, 2005. Second edition. Version 2.1, Corrected third printing.

[PZ07] S. Peszat and J. Zabczyk. Stochastic partial differential equations with Lévy noise, volume 113 of Encyclopedia of Mathematics and its Applications. Cambridge University Press, Cambridge, 2007. An evolution equation approach. 
[Roz90] B. L. Rozovskiǔ. Stochastic evolution systems, volume 35 of Mathematics and its Applications (Soviet Series). Kluwer Academic Publishers Group, Dordrecht, 1990. Linear theory and applications to nonlinear filtering, Translated from the Russian by A. Yarkho.

[RZ07] Michael Röckner and Tusheng Zhang. Stochastic evolution equations of jump type: existence, uniqueness and large deviation principles. Potential Anal., 26(3):255-279, 2007.

[Tin77] E. Tinfavičius. Linearized stochastic equations of nonlinear filtering of random processes. Lithuanian Mathematical Journal, 17(3):321-334, 1977-07011977.

[Ver12] Mark Veraar. The stochastic Fubini theorem revisited. Stochastics, 84(4):543$551,2012$.

[Wal86] John B. Walsh. An introduction to stochastic partial differential equations. In École d'été de probabilités de Saint-Flour, XIV-1984, volume 1180 of Lecture Notes in Math., pages 265-439. Springer, Berlin, 1986.

[Zha13] Xicheng Zhang. Degenerate irregular SDEs with jumps and application to integro-differential equations of Fokker-Planck type. Electron. J. Probab., 18:no. 55, 25, 2013.

[Zho13] Guoli Zhou. Global well-posedness of a class of stochastic equations with jumps. Adv. Difference Equ., pages 2013:175, 23, 2013. 